\title{
QUANTUM CORRECTIONS TO BARYON PROPERTIES IN CHIRAL SOLITON MODELS ${ }^{1}$
}

\author{
F. Meier and H. Walliser \\ Fachbereich Physik, Siegen University, \\ 57068 Siegen, Germany
}

\footnotetext{
${ }^{1}$ PACS 14.20.-c, 12.40.-y
} 


\begin{abstract}
Chiral lagrangians as effective field theories of $Q C D$ are successfully applied to meson physics at low energies in the framework of chiral perturbation theory. Because of their nonlinear structure these lagrangians allow for static soliton solutions which may be interpreted as baryons. Their semiclassical quantization, which provides the leading order in an $1 / N_{C}$ expansion $\left(N_{C}\right.$ is the number of colors) turned out to be insufficient in many cases to obtain good agreement with empirical baryon observables. However with $N_{C}=3$, large corrections are expected in the next-to-leading order which is carried by pionic fluctuations around the soliton background. The calculation of these corrections requires renormalization to 1-loop of the underlying field theory. We present a procedure to calculate the 1-loop contributions for a variety of baryonic observables. In contrast to chiral perturbation theory, terms with an arbitrary number of gradients may in principle contribute and the restriction to low chiral orders can only be justified by the investigation of the scale independence of the results. The results generally give the right sign and magnitude to reduce the discrepancy between theory and experiment with one exception: the axial quantities. These suffer from the fact that the underlying current algebra mixes different $N_{C}$ orders, which suggests a large and positive next-to-next-to-leading order contribution, which is probably sufficient to close the gap to experiment.
\end{abstract}




\section{Contents}

1 Introduction 3

1.1 Preliminaries . . . . . . . . . . . . . . . . . . . 3

1.2 The Skyrme model . . . . . . . . . . . . . . . . . . . . . . . . 5

1.3 Baryon ChPT vs. soliton picture: a comparison . . . . . . . . 8

2 General framework 11

2.1 Quantum corrections versus 1-loop corrections in the soliton

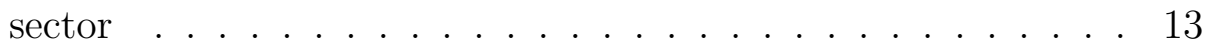

2.1 .1 Collective coordinates . . . . . . . . . . . . 13

2.1 .2 Continuum contributions: The phaseshift formula . . . 14

2.1 .3 External fields . . . . . . . . . . . . . . . . . . . . . . . 18

2.1.4 Treatment of zero modes in the presence of external fields . . . . . . . . . . . . . . . 20

2.2 Chiral lagrangian in the soliton sector . . . . . . . . . . . . . . 22

2.2 .1 Contributions from scalar mesons . . . . . . . . . . 24

2.2 .2 Contributions from vector mesons . . . . . . . . . . . . 24

2.3 One-loop corrections for lagrangian with explicit vector and scalar mesons . . . . . . . . . . . . . . . . . . . . . . . 29

$\begin{array}{lll}3 & \text { Baryon properties } & 32\end{array}$

B.1 Baryon mass . . . . . . . . . . . . . . . . . . . . . . . . 33

3.1 .1 Tree approximation . . . . . . . . . . . . . . . . 33

3.1 .2 Loop corrections and scale dependence of the soliton

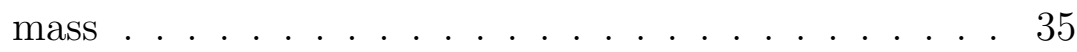

3.2 Scalar properties . . . . . . . . . . . . . . . . 39 
3.2 .1 Tree approximation . . . . . . . . . . . . . . . . . . 39

3.2 .2 Loop corrections to the scalar formfactor . . . . . . . . 40

3.3 Axial properties . . . . . . . . . . . . . . . . . . . . . . . . 41

3.3.1 Tree approximation . . . . . . . . . . . . . . . . . . 43

3.3.2 Loop corrections to the axial formfactor . . . . . . . . 44

3.3 .3 Current algebra and $1 / N_{C^{-} \text {expansion }}$. . . . . . . . . . 45

3.4 Electromagnetic formfactors . . . . . . . . . . . . . . . . . 48

3.4 .1 Tree approximation . . . . . . . . . . . . . . . . 50

3.4 .2 Loop corrections to the isovector magnetic formfactor . 51

3.4 .3 Loop corrections to the isoscalar formfactor . . . . . . 52

3.5 Polarizabilities . . . . . . . . . . . . . . . . . . . 52

3.5 .1 Electric polarizability in tree approximation . . . . . . 54

3.5.2 Loop corrections to the electric polarizability . . . . . . 57

$3.5 .3 \quad$ Neutron-proton split of the electric polarizability . . . 59

3.5 .4 Magnetic polarizability . . . . . . . . . . . . 62

$3.6 \quad$ Electromagnetic properties of the $\Delta$ isobar . . . . . . . . . . . 67

3.6 .1 Magnetic and quadrupole moments . . . . . . . . . . . 68

$3.6 .2 \quad E 2 / M 1$ ratio of the $\Delta$ photo-decay amplitudes . . . . . 69

\begin{tabular}{lll}
\hline 4 & Results & 73
\end{tabular}

\begin{tabular}{|lll}
\hline A & $1 / N_{C}$ contribution to $g_{A}$ & 86
\end{tabular}

A.1 Contribution due to rotationally induced soliton deformations 87

A.2 Contribution due to nonadiabatic loops . . . . . . . . . . . . . 89 


\section{Chapter 1}

\section{Introduction}

\subsection{Preliminaries}

During the past 15 years, effective field theories have been proven to be valuable tools in different branches of physics. In hadron physics, the need for effective field theories is brought about by the intractability of $Q C D$ at low energies around $1 \mathrm{GeV}$. Of course, the distinction between $Q C D$ on the one hand and effective field theories on the other is somewhat artificial at the conceptual level since $Q C D$ itself is probably an effective field theory of something more fundamental. However, for practical purposes, it is clear that the examples of effective field theories we are considering, namely chiral lagrangians formulated in terms of pseudoscalar fields, are much more restricted in their domain of validity and richness of phenomena they can describe than $Q C D$.

The most prominent, i. e. energetically lowest lying, degrees of freedom of such hadron effective field theories are pions (and kaons) thought to be the goldstone modes of the spontaneously broken chiral symmetry of $Q C D$. What makes these mesonic effective field theories tractable in a concise fashion is the concept of chiral perturbation theory $(C h P T)$, pioneered by Weinberg [1]-[3] and for the first time exploited by Gasser and Leutwyler [4] [5], which has been successfully applied to a variety of meson- and meson-baryon phenomena (for the latter case, see e.g. [6]). ChPT allows to calculate processes in a way that starting from a meson lagrangian $\mathcal{L}$ systematically includes all relevant terms up to some power of external momenta, which correspond to the number of derivatives (chiral order) contained in the vertices generated from $\mathcal{L}$, and the quark masses. The only theoretical input are universally accepted principles like chiral symmetry (and a certain pattern of symmetry breaking), Lorentz covariance etc. . The essential attraction of ChPT 
stems from the fact that calculations are no longer model dependent in the traditional sense. Unless some of the underlying general principles could be proven wrong, and as long as higher degrees of freedom (e.g. vector mesons) can safely assumed to be frozen, one only has to decide on the power in external momenta to which the calculation will be carried, and in this way the error introduced is under control.

The price to pay for this conceptual stringency rests with the fact that even at small orders, there is a vast number of independent terms in the effective lagrangian, each comes along with its own low energy constant $(L E C)$ to be fixed from experimental information. Since experimental knowledge, as well as the practical ability to include terms in a calculation are both limited, so is the applicability of $C h P T$.

Baryons have been introduced into the overall framework of effective field theories in several different ways: One possibility, directly related to $C h P T$, is to treat them as external fields coupled in the most general chiral invariant manner to pions. A similar momentum (and quark mass) power counting like the one outlined above may then be employed to calculate the 'dressing' of the originally pointlike particle by meson loops, provided the baryon is treated as a heavy field $(H B C h P T)$ [7]. Unfortunately, in this form, the proliferation of terms allowed from chiral symmetry alone already at the lowest nontrivial chiral orders is even more worrisome than in the purely mesonic case, and consequently the applicability of $H B C h P T$ is restricted even further compared to meson $C h P T$. is lost.

Therefore, the idea to create the baryons as solitons from the meson fields themselves without explicit fermionic degrees of freedom has been considered an especially attractive alternative, which has the advantage that one might directly build upon the effective lagrangian established in $C h P T$ in the mesonic sector. It is this aspect that defines the scope of our paper, namely, to investigate the extent to which the concept of baryons as solitons in effective meson theories can be linked to the methods and results of purely mesonic ChPT. Evidently, the consideration of loop corrections will be the crucial link in this connection.

In order to provide the reader with some motivation of what follows in the course of our report, we shall in this introduction recall the state of affairs in topological soliton models, outline the problems one has to face in trying to go beyond this status quo, and shed some light on the relationship between (baryon) ChPT and the soliton model calculation we are going to consider. 


\subsection{The Skyrme model}

The Skyrme model [8], invented about 35 years ago, yet reconsidered only within the timeframe mentioned in the beginning, emerges from the realization that nonlinear effective meson theories allow for topologically nontrivial static solutions (solitons) whose conserved topological index is then tentatively interpreted as the baryon number. Spin and isospin of the soliton are supplied by quantizing the zero modes of the problem. The reasons which left this model dormant for about 20 years after its inception (except for it being somehow orthogonal to the mainstream developments in medium energy physics) were essentially twofold: Firstly, in the absence of a suitable expansion parameter there was no compelling reason why zero modes should be preferred over other fluctuations in the soliton's quantization. Secondly, there was no argument to constrain the soliton to have half integer spin. The solution to both these problems is of course well known: The difficulty with half integer spin could be removed upon consideration of the Wess-ZuminoWitten anomaly, whereas the work of t'Hooft [9], [10] and Witten [11] on the expansion of $Q C D$ in powers of the inverse number of colours $1 / N_{C}$, besides making the concept of solitonic baryons more palatable as a low energy approximation to $Q C D$, showed that the pion-baryon coupling is suppressed in $1 / N_{C}$ (pions being identified with non-zero mode fluctuations of the soliton), and in this guise saved the zero mode quantization as a first approximation for baryon properties.

Since we will be concerned with corrections to this approximation, it is at this point worthwhile to recall some results given in reference [11]. There, it was shown that the $Q C D$ coupling constant scales like $N_{C}^{-1 / 2}$ if one assumes a smooth large $N_{C}$ limit for the 1-loop gluon self energy graph which would give rise to the worst divergences in $N_{C}$. Essentially from that result it follows that the pion baryon coupling must be of $\mathcal{O}\left(N_{C}^{1 / 2}\right)$, whereas the baryon mass is of $\mathcal{O}\left(N_{C}\right)$. Provided one accepts the conclusions of [11], one can calculate many observables to leading $\mathcal{O}\left(N_{C}\right)$, and since the picture of meson physics predicting baryon physics seemed rather appealing, the cited references consequently triggered a plethora of papers on the Skyrme model and its generalizations, examples of which are [12]-[26]. The leading contributions in $1 / N_{C}$ to static baryon properties, formfactors and pion nucleon scattering were calculated. What marred these calculations and what made the 'model' character of the Skyrme model was the difficulty to establish a firmly posed lagrangian from which to start, since the $1 / N_{C}$ expansion gives no argument as to the general form of $\mathcal{L}$. This meant that terms in $\mathcal{L}$ were mostly chosen for computational convenience and carried adjustable parameters. The resulting possibility to 'tailor' the lagrangian in order to obtain a 
best value for some quantity under consideration makes a clear judgement of the merits and defects of the Skyrmion approach to baryon physics difficult, because most calculations were limited to one or a few baryon observables for which the model used could be fine tuned. Since we want to provide the reader with a motivation for our project, we shall now investigate in some detail the status of several quantities within the Skyrme model.

In the following, we present a list of quantities which have been calculated in $S U(2)$ Skyrme-type models. Although even this fairly long list is not exhaustive, it may serve as a yardstick against which the quality of the model can reliably be measured, and which clearly illustrates the problems mentioned above. Therefore, in table 1.1 we present entries obtained from the standard Skyrme model with one free parameter $e$, in one case adjusted to give the best possible overall picture of baryon properties and in a second case chosen in order to cure already at leading $\mathcal{O}\left(N_{C}\right)$ what was perceived to be a major shortcoming, namely the too large soliton mass. It should be evident, that in the latter case, optimization of one value can only be achieved at the expense of all others. Eventually, we have included in table 1.1 ranges of the results for the different quantities as can be found in the skyrmion literature.

Table 1.1: Tree contribution to various quantities for the standard Skyrme model with various values of the Skyrme parameter $e$. Contained is one column that lists typical ranges for these quantities as found in the skyrmion literature.

\begin{tabular}{|c|c|c|c|c|}
\hline & $e=4.25$ & $e=7.3$ & $\begin{array}{c}\text { Skyrmion } \\
\text { Literature }\end{array}$ & exp. \\
\hline$M[\mathrm{MeV}]$ & 1648 & 941 & $1300-2100$ & 939 \\
\hline$\sigma[\mathrm{MeV}]$ & 42 & 11 & $30-60$ & $45 \pm 7$ \\
\hline$<r^{2}>^{S}\left[\mathrm{fm}^{2}\right]$ & .98 & .56 & $\simeq 1$ & $1.6 \pm .3$ \\
\hline$g_{A}$ & .90 & .32 & $.8-1.1$ & 1.26 \\
\hline$<r^{2}>_{A}\left[\mathrm{fm}^{2}\right]$ & .09 & .06 & $\simeq .1$ & $.42_{-.08}^{+.18}$ \\
\hline$<r^{2}>_{E}^{S}\left[\mathrm{fm}^{2}\right]$ & .61 & .47 & $.5-.8$ & .59 \\
\hline$\mu^{V}$ & 1.6 & .47 & $1.5-2.0$ & 2.35 \\
\hline$\mu^{S}$ & .18 & .29 & $.15-.25$ & .44 \\
\hline$<r^{2}>_{M}^{V}\left[\mathrm{fm}^{2}\right]$ & .70 & .40 & $.5-.7$ & .73 \\
\hline$\alpha\left[10^{-4} \mathrm{fm}^{3}\right]$ & 17.0 & 2.3 & $14-22$ & $9.5 \pm 5$ \\
\hline
\end{tabular}

Some examples from this list are 
- the nucleon mass as a prominent failure of the Skyrme model, since it comes out too large by a factor of almost 2 regardless of the detailed lagrangian used. An early effort to reproduce the experimental value in leading $\mathcal{O}\left(N_{C}\right)$ [12 required the reduction of $f_{\pi}$ to one half of its experimental value.

- the $\boldsymbol{\sigma}$ term, where the picture is less clear. Depending on the model used, it is over or underestimated. However, this quantity, extracted from the isospin even part of the $\pi \mathrm{N}$ scattering amplitude has also seen a considerable reduction of its experimental value according to the latest $\sigma$-term update [27]. The comparably large error bar is partly due to inconsistencies in the available data and partly due to some model dependence in the data analysis.

- the axial vector constant which is a weak point of Skyrme type models, too. It is an excellent example for the statement that adjusting the model to reproduce one quantity exactly is very much of a mixed blessing with respect to all others.

- the electromagnetic formfactors, where it has long been recognized that magnetic moments generally come out too small, most notably the isoscalar one, which is wrong by a factor of 2 , but also the isovector moment for which our parameter gives 1.6 nuclear magnetons in contrast to the experimental finding of 2.35. Interestingly, the defective $\mu^{S}$ has been impossible to repair even with some weird choice of parameters.

- the static electric polarizability, which is generally too large if one tries to obtain an overall good fit to the data for other properties as well. However, there has been some confusion in the literature concerning the exact status of this quantity in soliton models which we will have to discuss in more depth later on (section 3.5).

From the preceeding discussion, two points should be obvious: There is a definite need for improvements in the numerical values of several static quantities if the soliton picture is to proceed from a qualitative to a more quantitative description of the baryon, and such improvements can only come from the consideration of higher orders in $1 / N_{C}$. Because the next to leading order contribution in $1 / N_{C}$ is carried by pionic fluctuations, this involves calculation of pion loops, with all associated problems of regularization and renormalization of what is, strictly speaking, a nonrenormalizable theory. The second point is that it would be highly desirable to reduce the amount of arbitrariness in the model, preferably by establishing a tighter connection to meson physics. 
On the other hand, in view of the obvious problems of Skyrme-type models, one might ask why a deeper investigation of the soliton picture is at all worthwhile, since $H B C h P T$ seems to provide a systematic way to calculate baryon properties. This point of view, with which we definitely disagree, nevertheless deserves a closer look.

\subsection{Baryon ChPT vs. soliton picture: a com- parison}

As mentioned above, ChPT, for mesons as well as baryons, is an expansion in powers of external momenta and quark masses (plus inverse baryon mass in case of $H B C h P T$ ) without any reference to $N_{C}$ as an expansion parameter. Nevertheless, the $N_{C}$ counting rules outlined above apply generally, not just in solitonic theories, and one could look into their consequences for theories coupling external baryons to pions. This in fact has been accomplished [28], [29]. The picture that emerges is the following: Suppose one starts with a meson-baryon lagrangian of the form $\mathcal{L}=\mathcal{L}_{M}+\mathcal{L}_{M B}$ where the subscripts denote the purely mesonic part and the meson baryon interactions, and wherein the baryon has been introduced as an external field and provided $\mathcal{L}_{M}$ supports a stable soliton, then the leading $\mathcal{O}\left(N_{C}\right)$ of this solitonic theory corresponds to the set of all multi loop graphs without a closed meson loop generated from $\mathcal{L}$ and evaluated in the limit of a static baryon. (As a shorthand, and in order to maintain the distinction between solitonic theory and $H B C h P T$, we shall furtheron call ChPT loops the ones generated by $\mathcal{L}$.) Although, from refs [28] [29], this connection is not strictly proven, it nevertheless seems very plausible. With the same comment, one might go one step further and state that the 1-loop level of the soliton model corresponds to the set of multi-loop graphs deduced from $\mathcal{L}$ containing one closed pion loop and evaluated in heavy baryon approximation.

The calculational scheme provided by (baryon) $C h P T$ now ties the number of loops to which one carries the calculation to the specific form of $\mathcal{L}$ : The more $C h P T$ loops, the more complicated becomes $\mathcal{L}$. Stated differently, once one has chosen $\mathcal{L}$, one has a limit on the number of $C h P T$ loops to which the calculation might be consistently extended. For an infinite number of loops, $\mathcal{L}$ would have to contain an infinite number of terms.

Two consequences follow from these remarks: Provided the picture about the connection of $H B C h P T$ and soliton models outlined in 28] 29] is correct, the leading $N_{C}$ part of the $C h P T$ 1-loop contribution must be contained in the tree level of a soliton model. We shall demonstrate this in the course of our report on several occasions. The other consequence is that a soliton 
model must be accompanied by an additional assumption not inherent in ChPT to justify working with a lagrangian that consists of a finite number of terms despite summing an infinite number of $H B C h P T$ loops already at leading $\mathcal{O}\left(N_{C}\right)$.

The practicality of a project involving the calculation of loop corrections to baryon observables in soliton models therefore hinges upon the answers to the following questions:

- Can we justify the choice of a particular chiral lagrangian used to construct the soliton, which of necessity must be truncated to some finite chiral order, by arguments other than small-derivative-ones?

- Once this general form of lagrangian then has been established, can we make contact to meson phenomenology upon using experimental LECs within the theory, and have good values for all baryon properties?

In the course of this report we shall try to answer these questions. In order to maintain the tightest possible connection to the meson sector, we will show that it is necessary to use a lagrangian that is different from the ones found in the skyrmion literature. Before entering the calculation of loop corrections, we have to recalculate the entire set of tree level quantities from this lagrangian, mainly because all tree quantities should be calculated using the same lagrangian and the same parameter combination, and because we found several earlier tree level calculations to be in need for improvements, specifically those for the magnetic polarizability and the electromagnetic ratio of the $\Delta$ photodecay amplitudes. Additionally, we calculated the neutron proton split of the electric polarizability, which had not been derived in soliton models before.

For these reasons and also in order to clarify the relationship between 1loop calculations in the meson and the soliton sector, large parts of the paper are of review character.

This report is organized as follows: Chapter 2 provides the general framework for the calculations, namely a brief review of ChPT (section 2.1), the quantization of the soliton and its renormalization in the presence of those external fields related to the baryon quantities of interest (section 2.1) as well as the set up of the lagrangian (section 2.2). Chapter 3 then presents the investigations for specific baryon observables, in detail mass (section 3.1), scalar properties (section 3.2), axial properties (section 3.3), electromagnetic formfactors (section 3.4) and polarizabilities (section 3.5) and electromagnetic properties of the $\Delta$ resonance (section 3.6). Results are summarized and discussed in chapter 4 . In this chapter we also draw some conclusions 
concerning several problems which occured during the process of the calculations to finally return to the questions posed in the beginning. 


\section{Chapter 2}

\section{General framework}

As pointed out in the introduction, effective field theories are designed to describe more fundamental theories (which may well be again effective) at low energies where only part of the degrees of freedom are important. In the standard model these effective degrees of freedom are assumed to be mesons constrained by the requirement of global chiral symmetry which is spontaneously broken into a non chirally invariant vacuum state. Restriction to the lowest-lying meson states which may be interpreted as Goldstone modes of the broken symmetry leads to the lagrangian

$$
\mathcal{L}_{e f f}(U)=\mathcal{L}^{(2)}+\mathcal{L}^{(4)}+\ldots \mathcal{L}^{(N)}+\ldots
$$

where the $C h O N$ counts the number of gradients contained in each term $\mathcal{L}_{i}^{(N)}$ of

$$
\mathcal{L}^{(N)}=\sum_{i} \ell_{i}^{(N)} \mathcal{L}_{i}^{(N)}
$$

which comes with an $L E C$ to be fixed from experimental information. For convenience $U$ is here an $S U(2)$ matrix

$$
U=e^{i \boldsymbol{\tau} \boldsymbol{\pi} / f_{\pi}} \in S U(2)
$$

which depends nonlinearly on the pion field. The restriction to pionic degrees of freedom is of course meaningful only below energies where the next higher resonances become important. For example, starting from a theory which includes explicit vector meson degrees of freedom we could replace the vector through pseudoscalar degrees of freedom by virtue of a gradient expansion in powers of the inverse vector meson mass $m_{V}$. This would formally generate no terms not already present in (2.1), yet make sense numerically only if the gradients involved were smaller than $m_{V}$.

A loop expansion of a theory like (2.1) necessarily brings about the need for renormalization. Provided the regularization procedure used respects chiral 
symmetry, then (2.1) is renormalizable in the sense that no counterterm can be produced which is not present already. However, this statement is rather academical because for practical reasons it is impossible to treat $\mathcal{L}_{\text {eff }}$ as a whole, instead it must be truncated to some finite number of terms preferably at some finite chiral order. The question is of course whether this truncation can be justified.

For the answer, we have to distinguish the vacuum sector from the soliton sector. Generally, Weinberg [1] realised that matrix elements of (2.1) behave like some power $p$ of external momenta carried by the source fields. In a graph containing $n_{N}$ vertices with $N$ derivatives this power is related to the number of loops $n_{L}$

$$
p=2 n_{L}+2+\sum_{N} n_{N}(N-2)
$$

One loop graphs from the $N \ell \sigma$ model $\mathcal{L}^{(2)}$ always come along with a power $p=4$ irrespective of the number of their vertices and the divergencies can always be absorbed into the tree graph coefficients of $\mathcal{L}^{(4)}$. Proceeding to higher chiral orders this situation changes fundamentally. For example already one loop graphs from $\mathcal{L}^{(4)}$ produce all powers $p=4+2 n_{4}$ depending on the number of vertices $n_{4}$, and consequently their renormalization affects the coefficients of tree graphs to all chiral orders in (2.1).

The characteristic feature of the meson sector now is that external momenta can be made small by construction i.e. by designing the experiment such that this requirement is fulfilled. The truncation of $\mathcal{L}$ in (2.1) is then justified, with ChPT being the result. The crucial difference in the soliton sector is that the soliton itself constitutes "external" fields, which cannot be made weak by assumption. In fact, gradients of the soliton profiles are typically of the order of $700 \mathrm{MeV}$ which is dangerously close to the scale of $m_{V}$. Therefore, in the soliton sector, we can neither disregard the counterterms of any $p$ generated by a 1-loop graph nor can we, at first glance, get rid of multi-loop graphs.

For the latter problem there is a way out. Following Witten [11, higher loops are suppressed by additional powers of the inverse number of colours $N_{C}$. Thus, for a first correction to the leading tree approximation in the soliton sector, we consider the 1-loop contribution to be satisfactory. In contrast to this, the problem of counterterms to all chiral orders may only be solved by an ad hoc assumption, namely we must assume that the renormalized $L E C s$ of higher chiral orders are small. Whether this assumption is justified will be investigated during the course of this report. 


\subsection{Quantum corrections versus 1-loop cor- rections in the soliton sector}

As discussed above, in the meson sector, the calculation of quantum corrections, next-to-leading order in $N_{C}$, corresponds to evaluating the set of 1-loop graphs generated by the chiral lagrangian. This is different in the soliton sector. There, the existence of a nontrivial static solution, the hedgehog

$$
U_{0}=e^{i \boldsymbol{\tau} \hat{\boldsymbol{r}}_{F(r)}}
$$

means that the classical "vacuum" field configuration is no longer invariant with respect to the rotational and translational symmetries of the lagrangian. Instead, one has a set of degenerate "vacua" connected to each other by these global symmetry transformations.

A fluctuation which parametrizes such a transformation doesn't remain small in the course of its time evolution and therefore has to be treated to all orders. Technically, this amounts to introducing collective coordinates [30]. It was one of the central assumptions in the early paper on baryon properties by Adkins, Nappi and Witten [12] as well as in Skyrme's original work [8] that the quantization of these collective modes already supplies a reasonable approximation. In this sense we should distinguish in the soliton sector the quantum corrections due to collective degrees of freedom, which have been taken into account from the earliest attempts on, from quantum corrections caused by 1-loop diagrams in which interest emerged much later [21], [31][36].

\subsubsection{Collective coordinates}

As a prelude to the calculation of 1-loop corrections, we shall briefly recapitulate the collective coordinate method used in the soliton sector [12]. We introduce the position $\boldsymbol{X}(t)$ of the soliton centre and an $S U(2)$ matrix $A(t)$ which parametrizes the soliton's isorotation through three time dependent Euler angles

$$
U(\boldsymbol{x}, t)=A(t) U_{0}(\boldsymbol{x}-\boldsymbol{X}(t)) A^{\dagger}(t) .
$$

Due to the peculiar structure of the hedgehog solution (2.5) a rotation in isospace is equivalent to a rotation in coordinate space. With the angular velocities $A^{\dagger} \dot{A}=\frac{i}{2} \tau \Omega^{R}$ one obtains

$$
\dot{U}=A\left(\frac{i}{2}\left[\tau \Omega^{R}, U_{0}\right]-\dot{\boldsymbol{X}} \nabla U_{0}\right) A^{\dagger}
$$


and the lagrangian

$$
L=-M_{0}+\frac{1}{2} M_{0} \dot{\boldsymbol{X}}^{2}+\frac{1}{2} \Theta\left(\Omega^{R}\right)^{2}
$$

in terms of the soliton mass $M_{0}$ and the moment of inertia $\Theta$ to be specified later. For the derivation of (2.8) slow motion of the soliton has been assumed. With the angular and linear momenta

$$
\begin{aligned}
R_{a} & =-\frac{\partial L}{\partial \Omega_{a}^{R}} \\
L_{a} & =D_{a b} R_{b}, \quad D_{a b}=\frac{1}{2}\left\langle\tau_{a} A \tau_{b} A^{\dagger}\right\rangle \\
P_{a} & =\frac{\partial L}{\partial \dot{X}_{a}}
\end{aligned}
$$

$(\langle\rangle$ denotes the trace in isospace ) following the canonical quantization procedure

$$
\left[R_{a}, R_{b}\right]=-i \varepsilon_{a b c} R_{c}, \quad\left[L_{a}, L_{b}\right]=i \varepsilon_{a b c} L_{c}, \quad\left[P_{a}, X_{b}\right]=-i \delta_{a b}
$$

the hamiltonian is obtained as

$$
H=M_{0}+\frac{\boldsymbol{P}^{2}}{2 M_{0}}+\frac{\boldsymbol{R}^{2}}{2 \Theta}
$$

and for the construction of eigenstates with definite spin $\left(S, S_{3}\right)$ and isospin $\left(T, T_{3}\right)$ quantum numbers

$$
<\boldsymbol{x}, A \mid \psi_{T_{3}, S_{3}}^{T=S}(\boldsymbol{P}, t)>=\sqrt{\frac{2 T+1}{8 \pi^{2}}}(-)^{T-S_{3}} D_{T_{3},-S_{3}}^{T=S}(A) e^{i\left(\boldsymbol{P} \boldsymbol{X}_{-E t}\right)}
$$

$S U(2) D$-functions may be employed. The calculation of matrix elements with the states (2.12) for the various currents of interest will be explained in the corresponding sections.

\subsubsection{Continuum contributions: The phaseshift for- mula}

In order to quantize the continuum modes of the chiral lagrangian we consider the path integral [37]

$$
W=N \int d[U] e^{-i Z[U]}, \quad Z[U]=\int d^{4} x \mathcal{L}
$$


where $Z$ is the effective action and $N$ a normalization factor. Fluctuations are now introduced through the following ansatz [4]

$$
U=A \xi e^{i \boldsymbol{\tau} \boldsymbol{\eta}(\boldsymbol{x}-\boldsymbol{X}) / f_{\pi}} \xi A^{\dagger}, \quad \xi=\sqrt{U_{0}} .
$$

$A$ and $\boldsymbol{X}$ denote the collective coordinates introduced in the previous section. The next step then is to expand $\mathcal{L}$ to quadratic order in the fluctuations. In contrast to these fluctuations which come only with a factor $1 / f_{\pi}=\mathcal{O}\left(N_{C}^{-1 / 2}\right)$, time derivatives on collective coordinates count as $\mathcal{O}\left(N_{c}^{-1}\right)$ and may be neglected because first of all we are interested in the leading loop corrections only. Using this adiabatic approximation the path integral reads

$$
W=N \int d[A] d[\boldsymbol{X}] d[\boldsymbol{\eta}] e^{-i \int d^{4} x\left[\mathcal{L}\left(U_{0}, A, \boldsymbol{X}\right)+\frac{1}{2} \eta_{a} \Omega_{a b} \eta_{b}\right]} .
$$

For the decomposition of the measure $d[U]=d[A] d[\boldsymbol{X}] d[\boldsymbol{\eta}]$, orthogonality of the fluctuations on the collective modes has to be presumed in order to guarantee independence of the integration variables and also to avoid double counting. For that purpose, the zero modes have to be excluded from the space of allowed proper fluctuations. Formally, integrating over $\boldsymbol{\eta}$, the generating functional

$$
Z=\int d^{4} x \mathcal{L}\left(U_{0}, A, \boldsymbol{X}\right)+\frac{1}{2} \int d^{4} x\langle\ln \Omega\rangle
$$

contains a trace log of the operator governing the time evolution of the fluctuations. The task now is to evaluate this trace log and, in particular, to isolate the divergencies residing in it.

Here, one encounters the principal technical difficulty of a soliton sector calculation: The presence of the static soliton implies that axial, vector and (pseudo) scalar sources are contained in the equations of motion (e.o.m) for the fluctuations even in the absence of true external fields. These sources lead to a metric appearing in the kinetic part of the e.o.m

$$
\Omega_{a b}=-\partial_{t} n_{a b}^{2} \partial_{t}-h_{a b}^{2}
$$

Although the fact that the norm $n_{a b}^{2}$ is time independent simplifies matters somewhat, so far, we are not aware that anybody has been able to perform a heat kernel expansion for this kind of operator, which is the method used in [4] to find the residues of the poles in the trace $\log$ of $\Omega$.

Consequently, one has to resort to a different procedure. The alternative is the numerical determination of the divergencies from an exact diagonalization of the e.o.m for the fluctuations, which according to their time dependence $\sim e^{-i \omega t}$ may be written as

$$
h_{a b}^{2} \eta_{b}=\omega^{2} n_{a b}^{2} \eta_{b}
$$


For convenience fluctuations normalized with respect to a flat metric may be introduced

$$
\tilde{h}_{a b}^{2} \tilde{\eta}_{b}=\omega^{2} \tilde{\eta}_{b}, \quad \tilde{h}_{a b}^{2}=n_{a c}^{-1} h_{c d}^{2} n_{d b}^{-1}, \quad \tilde{\eta}_{a}=n_{a b} \eta_{b}
$$

the spectrum, or, more precisely, the eigenvalues and phaseshifts, are not affected by this transformation. If $\tilde{h}^{2}$ is time independent and does not contain time derivatives, one has

$$
\frac{i}{2} \int d^{4} x\langle\ln \Omega\rangle=-\frac{T}{2} \int d^{3} r\langle\tilde{h}\rangle,
$$

where $T$ limits the time integral. For a one-dimensional potential scattering problem, it can be shown 38 that the continuum part of the scattering operator's trace may be accounted for through a phaseshift integral. Using the hedgehog ansatz in the adiabatic approximation the e.o.m for the fluctuations (2.18) may be decomposed into partial waves 339 for which the one-dimensional phase shift formula applies. The total trace of the scattering operator is then obtained by replacing $\delta$ by the sum over phaseshifts of all angular momenta

$$
\begin{aligned}
\frac{1}{2} \int d^{3} r\left\langle\tilde{h}-h_{0}\right\rangle & =\frac{1}{2 \pi} \int_{m_{\pi}}^{\infty} d \omega \omega \frac{d}{d \omega} \delta(\omega) \\
& =\frac{1}{2 \pi} \int_{o}^{\infty} d p \sqrt{p^{2}+m_{\pi}^{2}} \frac{d}{d p} \delta(p) .
\end{aligned}
$$

Here, $h_{0}^{2}=-\Delta+m_{\pi}^{2}$ corresponds to the free Klein-Gordon equation in the absence of the soliton fields. In the last step the $\omega$ integration, $\omega=\sqrt{p^{2}+m_{\pi}^{2}}$, has been converted to a momentum integration. From (2.21) it is clear that divergencies are related to the high momentum behaviour of the phaseshifts 36

$$
\delta(p) \stackrel{p \rightarrow \infty}{\longrightarrow} a_{0} p^{3}+a_{1} p+\frac{a_{2}}{p}+\mathcal{O}\left(p^{-3}\right) .
$$

The explicitly denoted terms give rise to at least logarithmically divergent expressions. The strategy is now to subtract the worrisome terms in the phase shift integral and add them separately. Using dimensional regularization

$$
\begin{aligned}
\int \frac{d^{d-1} p}{(2 \pi)^{d-1}}\left(p^{2}+m_{\pi}^{2}\right)^{1 / 2} & =m_{\pi}^{4}\left(\lambda+\frac{1}{32 \pi^{2}}\left(-\frac{1}{2}+\ln \frac{m_{\pi}^{2}}{\mu^{2}}\right)\right) \\
\int \frac{d^{d-1} p}{(2 \pi)^{d-1}}\left(p^{2}+m_{\pi}^{2}\right)^{-1 / 2} & \left.=4 m_{\pi}^{2}\left(\lambda+\frac{1}{32 \pi^{2}} \ln \frac{m_{\pi}^{2}}{\mu^{2}}\right)\right) \\
\int \frac{d^{d-1} p}{(2 \pi)^{d-1}}\left(p^{2}+m_{\pi}^{2}\right)^{-3 / 2} & =-8\left(\lambda+\frac{1}{32 \pi^{2}}\left(1+\ln \frac{m_{\pi}^{2}}{\mu^{2}}\right)\right)
\end{aligned}
$$


which involves a scale $\mu$ to render the arguments in the logarithms dimensionless, the poles as $d \rightarrow 4$ which reside in

$$
\lambda=\frac{\mu^{d-4}}{16 \pi^{2}}\left(\frac{1}{d-4}-\frac{1}{2}\left(\Gamma^{\prime}(1)+\ell n(4 \pi)+1\right)\right)
$$

may be isolated

$$
\frac{1}{2} \int d^{3} r\left\langle\tilde{h}-h_{0}\right\rangle=E_{\text {cas }}(\mu)+\lambda\left(3 \pi m_{\pi}^{4} a_{0}-4 \pi m_{\pi}^{2} a_{1}+8 \pi a_{2}\right) .
$$

The Casimir energy

$$
\begin{aligned}
E_{c a s}(\mu) & =\frac{1}{2 \pi}\left(\int_{0}^{\infty} \frac{d p}{\sqrt{p^{2}+m_{\pi}^{2}}}\left[-p\left(\delta(p)-a_{0} p^{3}-a_{1} p\right)+a_{2}\right]-m_{\pi} \delta(0)\right. \\
& \left.+\frac{m_{\pi}^{4} a_{0}}{16}\left(\frac{1}{2}+3 \ln \frac{m_{\pi}^{2}}{\mu^{2}}\right)-\frac{m_{\pi}^{2} a_{1}}{4} \ln \frac{m_{\pi}^{2}}{\mu^{2}}+\frac{a_{2}}{2}\left(1+\ln \frac{m_{\pi}^{2}}{\mu^{2}}\right) a_{2}\right)
\end{aligned}
$$

is a finite but scale-dependent expression. For the derivation of eqs.(2.25, 2.26) an integration by parts has been performed and $m_{\pi}^{2}$ has been added to the denominator of the $a_{2}$ term in order to avoid infrared problems. The coefficients $a_{0}, a_{1}$ and $a_{2}$ contain in general all $C h O s$ as is noticed from the fact that $\tilde{h}=\sqrt{h_{0}^{2}+\tilde{w}}$ with some potential $\tilde{w}$. Therefore the trace $(2.25)$ contains not only all ChOs via the soliton's stability condition but also an infinite numer of explicit gradients. The part proportional to the divergent $\lambda$ may formally be expanded

$$
3 \pi m_{\pi}^{4} a_{0}-4 \pi m_{\pi}^{2} a_{1}+8 \pi a_{2}=\sum_{i, N} \gamma_{i}^{(N)} \int d^{3} r \mathcal{L}_{i}^{(N)}, \quad N \geq 4
$$

into the complete set of terms $\mathcal{L}_{i}^{(N)}$ contained in the chiral lagrangian (2.1). The coefficient $\gamma_{i}^{(N)}$ for a definite term of $C h O N$ is in general complicated and scale dependent through all the renormalized LECs of $C h O$ smaller than $N$. Again, it is only the 1-loop contribution to the $N \ell \sigma$ model which corresponds to the lowest non-vanishing $\mathrm{ChO} 4$ in (2.27), where the situation becomes simple. For that particular case $\left(a_{0}^{(0)}=0\right) \quad a_{1}^{(2)}$ and $a_{2}^{(4)}$ are analytically known [- 34 and the coefficients $\gamma_{i}^{(4)}$ are simple numerical factors (section 2.2).

According to (2.27) the divergencies in (2.25) may finally be absorbed into a redefinition of the lagrangians LECs. The total soliton mass (tree + 1-loop) is obtained as

$$
\begin{aligned}
M(\mu) & =-\sum_{i, N}\left(\ell_{i}^{(N)}-\gamma_{i}^{(N)} \lambda\right) \int d^{3} r \mathcal{L}_{i}^{(N)}+E_{\text {cas }}(\mu) \\
& =M_{0}\left(\ell_{i}^{(N) r}\right)+E_{c a s}(\mu)
\end{aligned}
$$


where $E_{\text {cas }}$ represents the Casimir energy (2.26) and

$$
M_{0}\left(\ell_{i}^{(N) r}\right)=-\sum_{i, N} \ell_{i}^{(N) r} \int d^{3} r \mathcal{L}_{i}^{(N)}
$$

is the tree contribution to the soliton mass calculated with the renormalized $L E C s$

$$
\ell_{i}^{(N) r}=\ell_{i}^{(N)}-\gamma_{i}^{(N)} \lambda .
$$

It is this relation which for $N=4$ establishes the close connection to ChPT in the meson sector with the conventions adopted by Gasser and Leutwyler. With $\lambda$ defined in (2.24) the renormalized LECs $\ell_{i}^{(4) r}$ (2.30) just coincide with those defined in [4] and auspiciously we may take over their analysis (section 2.2).

According to (2.30) the renormalized LECs for the higher ChOs, in particular their scale dependence, are fixed in principle, however for practical purposes we do not know the $\gamma_{i}^{(N)}$ for the individual higher $C h O$ terms. Even if we knew the value of the corresponding renormalized $L E C$ at some scale we could not, according to (2.30) determine its scale dependence without knowledge of the $\gamma_{i}^{(N)}$. For a detailed examination of the scale dependence of the soliton's mass see section 3.1.2.

As mentioned, the phaseshift formula (2.21) takes care of the continuum only; if there exist any bound states at energies $\omega_{c}$ the zero point energy $\frac{1}{2} \sum_{c} \omega_{c}$ has to be added to the Casimir energy. In the absence of external fields, the spectrum of $h^{2}$ contains no true bound states, instead there exist zero modes due to the rotational and translational symmetries discussed in section 2.1 . Because of $\omega_{c}=0$ the zero modes do not explicitly contribute. However, in the presence of external fields, which violate rotational or translational symmetries, zero modes are shifted to finite energies. Their treatment is described in section 2.1.4.

\subsubsection{External fields}

The above formulae allow to calculate the static soliton's energy including quantum corrections due to pion loops. However, the aim of this investigation is to calculate other baryon properties on the same footing, too. Such quantities may be defined as the linear change of the baryon's energy in the presence of an external stimulus.

To be specific, we study the coupling of an external field $j$ with strength $\varepsilon$ to the lagrangian (2.1)

$$
\mathcal{L}(\varepsilon)=\mathcal{L}\left(\ell_{i}, U\right)-\varepsilon j \cdot J\left(\ell_{i}, U\right),
$$


where $J\left(\ell_{i}, U\right)$ denotes the corresponding current density. The external field has to be chosen suitably so as to give the desired quantity, e.g. for the calculation of the magnetic moment, $J$ represents the electromagnetic current density and $j \cdot J$ corresponds to the magnetic moment density. Matrix elements of $j \cdot J$ are then obtained as a derivative of the soliton mass (tree +1 loop) in the presence of the external field with respect to its strength

$$
\begin{aligned}
& \left\langle N\left|\int d^{3} r j J\right| N\right\rangle=\left.\frac{\partial M(\varepsilon)}{\partial \varepsilon}\right|_{\varepsilon=0} \\
& M(\varepsilon)=M_{0}\left(\varepsilon, \ell_{i}^{r}\right)+E_{\text {cas }}(\varepsilon, \mu) .
\end{aligned}
$$

Here, $|N\rangle$ represents the one baryon state (2.12) .

Considering static baryon properties, the external field can be specialised to a simple, in many cases space-time-independent form, and can generally be assumed to be weak. Consequently, we shall proceed as follows:

(i) The external field is chosen such that the quantity of interest is obtained from (2.32) in tree approximation.

(ii) The static soliton is computed from (2.31) for some small $\varepsilon$. It turns out that for all quantities considered here, the hedgehog still solves the classical e.o.m in the presence of the external field.

(iii) The operators $h^{2}(\varepsilon)$ and $n^{2}(\varepsilon)$ are obtained by expanding $\mathcal{L}(\varepsilon)$ to quadratic order in the fluctuations. The stability condition for the hedgehog (ii) ensures that the term linear in the fluctuations is absent.

(iv) The e.o.m for the fluctuations $h^{2}(\varepsilon) \eta=\omega^{2}(\varepsilon) n^{2}(\varepsilon) \eta(\varepsilon)$ in the presence of the external field is solved for the scattering phaseshift up to sufficiently large linear and angular momenta, respectively.

(v) The Casimir energy $E_{\text {cas }}(\mu, \varepsilon)$ is computed according to (2.26) and the soliton mass in tree +1 -loop is obtained in the presence of the external field.

(vi) Repeating the whole procedure for several small values of $\varepsilon$, the derivative $\partial M(\varepsilon) /\left.\partial \varepsilon\right|_{\varepsilon=0}$ may finally be computed, which equals the desired quantity including quantum corrections.

We again stress that in this method the integral has to be restricted to the continuum fluctuations orthogonal on the zero modes. Whereas this orthogonality holds automatically in the absence of external fields, it generally has to be imposed in their presence (c.f. next section) . 
A second caveat concerns eq. (2.20) . If the external field creates a timederivative interaction as is the case for the electric polarizabilities (section $3.5)$

$$
\Omega=-\partial_{t} n^{2} \partial_{t}-2 i \varepsilon w \partial_{t}-h^{2}
$$

where $w\left(\right.$ and $\left.\tilde{w}=n^{-1} w n^{-1}\right)$ is time independent, we have

$$
\begin{aligned}
\frac{i}{2} \int d^{4} x\langle\ln \Omega\rangle & =i \frac{T}{2} \int d p_{0} \int d^{3} r\left\langle\ln \left(p_{0}^{2}-2 \varepsilon p_{0} \tilde{w}-\tilde{h}^{2}\right)\right\rangle \\
& =-\frac{T}{2} \int d^{3} r\left\langle\sqrt{\tilde{h}^{2}+\varepsilon^{2} \tilde{w}^{2}}\right\rangle
\end{aligned}
$$

i.e. there is no term linear in $\varepsilon$ and the leading contribution is of $\mathcal{O}\left(\varepsilon^{2}\right)$.

The specific choice of external field, as well as other details, will be given separately for each quantity in question within the corresponding subsection of the next chapter.

\subsubsection{Treatment of zero modes in the presence of ex- ternal fields}

Due to translational and rotational invariance the system of e.o.m for the adiabatic fluctuations possesses 6 zero mode solution $z_{a}^{c}$ with $\omega_{c}=0$

$$
h_{a b}^{2} z_{b}^{c}=0, \quad c=1 \ldots 6
$$

which enter the phaseshift formula for the Casimir energy only indirectly via Levinson's theorem $\delta(0)=6 \pi$. Because of $\omega_{c}=0$, there is no direct bound state contribution as e.g. in the case of the kink's breathing mode [30].

In the presence of external fields, the situation changes, since rotational and/or translational symmetries are in general violated. This is always the case if the external field coupled to the lagrangian is not rotationally or translationally invariant. If such a field is switched on weakly with strength $\varepsilon$, the zero modes of the corresponding broken symmetry are shifted to finite energies $\omega_{c} \sim \sqrt{\varepsilon}$. This is understood immediately, because the external field generates an additional term in the e.o.m for the fluctuations $h^{2}(\varepsilon)=h^{2}+\varepsilon w$ which in perturbation theory leads to $\omega_{c}^{2}=\varepsilon \int d^{3} r z_{a}^{c} v_{a b} z_{b}^{c}$ if the matrixelement does not vanish because of rotational or translational invariance. For the case of the axial vector coupling constant $g_{A}$ and the corresponding external axial field which violates the former but not the latter symmetry (c.f. section 3.3 ) we calculated the energies of the rotational zero modes numerically for different field strengths $\varepsilon$. The result, plotted in fig. 2.1 illustrates the behaviour $\omega_{c} \sim \sqrt{\varepsilon}$ for the zero modes which, considered as true bound states 
with contribution $\frac{1}{2} \sum_{c} \omega_{c}$ to the Casimir energy would lead to a desaster: The corresponding baryon property $\partial M(\varepsilon) /\left.\partial \varepsilon\right|_{\varepsilon=0}$ would aquire infinite quantum corrections.

Figure 2.1: Energy of the rotational zero modes as function of the strength $\varepsilon$ of a constant external axial field. Because the external field violates rotational invariance, the zero modes are shifted to finite energies proportional to the square root of the external field strength $\varepsilon$.

The solution to this problem is obtained by removing the zero modes, which are not confined by a restoring force, from the space of allowed small amplitude fluctuations. In fact, their contribution has already been taken into account in tree approximation by the introduction of collective coordinates. To remove the redundant variables in order to avoid double counting we must impose constraints [40] (see also section 3.5 for details)

$$
\int d^{3} r z_{a}^{c} n_{a b}^{2} \eta_{b}=0, \quad \int d^{3} r z_{a}^{c} n_{a b}^{2} z_{b}^{d}=\delta_{c d}
$$

which have to be added with multipliers $\lambda_{c}$ to the lagrangian. Only then the Legendre transformation to the hamiltonian will be well behaved. Without external fields, the constraints (2.36) are automatically fulfilled and the calculation of the soliton's Casimir energy remains untouched. 
However, in the presence of symmetry breaking external fields, the constraints become active and contribute to the e.o.m

$$
h_{a b}^{2}(\varepsilon) \eta_{b}(\varepsilon)-\lambda_{c}(\varepsilon) n_{a b}^{2} z_{b}^{c}=\omega^{2} n_{a b}^{2}(\varepsilon) \eta_{b}(\varepsilon) .
$$

The dependence on $\varepsilon$ is indicated wherever necessary; for the constraint itself this dependence is irrelevant, since $\mathcal{O}(\varepsilon)$ is sufficient to calculate the baryon property $\partial M(\varepsilon) /\left.\partial \varepsilon\right|_{\varepsilon=0}$ of interest and $\lambda_{c}$ is already of $\mathcal{O}(\varepsilon)$ :

$$
\lambda_{c}=\int d^{3} r z_{a}^{c}\left[h_{a b}^{2}(\varepsilon)-\omega^{2} n_{a b}^{2}(\varepsilon)\right] \eta_{b}(\varepsilon) \sim \varepsilon
$$

In fact, we are going to demonstrate that apart from removing the zero mode bound states the effect of the constraints on the scattering states is $\mathcal{O}\left(\varepsilon^{2}\right)$ and may therefore safely be discarded.

For this purpose, we first solve the e.o.m (2.37) without constraints

$$
h_{a b}^{2}(\varepsilon) \bar{\eta}_{b}(\varepsilon)=\omega^{2} n_{a b}^{2}(\varepsilon) \bar{\eta}_{b}(\varepsilon)
$$

and then treat the constraints as perturbation calculating the DWBA matrix element which is related to the phaseshift change

$$
\triangle \delta(p)=p\left\{\int d^{3} r \bar{\eta}_{a} n_{a b}^{2}(\varepsilon) z_{b}^{c}\right\}\left\{\int d^{3} r z_{e}^{c}\left[h_{e d}^{2}(\varepsilon)-\omega^{2} n_{e d}^{2}(\varepsilon)\right] \bar{\eta}_{d}\right\} .
$$

Both integrals vanish linearly with $\varepsilon$ and the phaseshift change is clearly $\mathcal{O}\left(\varepsilon^{2}\right)$.

Thus the situation becomes quite advantageous in this respect: For the phaseshift calculation the constraints may be ignored and in the Casimir energy they just remove the unwanted contribution $\frac{1}{2} \sum_{c} \omega_{c}$. The phaseshift formula (2.25, 2.26) as it stands remains correct in the presence of external fields which violate rotational and/or translational symmetry.

\subsection{Chiral lagrangian in the soliton sector}

We now turn to the discussion of the lagrangian to be used in the soliton sector. Since for consistency reasons we have to solve the static e.o.m of the same lagrangian that generates the loop graphs, it is clear that such an object

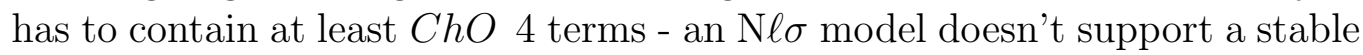
soliton. The question then is, what chiral order, if any, would be sufficient?

Recalling the remarks of section 2.1 we have to face the facts that counterterms will contain all chiral orders and, even more disturbingly, that an 
expansion in powers of external momenta must fail because of the size of gradients on the soliton field.

Consequently, it would be meaningless to simply count gradients in Weinberg-Gasser-Leutwyler fashion. Instead, either the renormalized $L E C s$ beyond some chiral order must be small or a cancellation between different terms must occur in order to assure negligible contributions.

The smallness of a renormalized $L E C$ is of course to some extent dependent on the choice of scale. But this choice is restricted in several ways: A pragmatic restriction stems from the requirement of a small symmetric 4th order term in $\mathcal{L}$ so as not to destroy the soliton. Another, more fundamental point is that the renormalized $L E C s$ must be adjusted to be predominantly of $\mathcal{O}\left(N_{C}\right)$ since otherwise the $1 / N_{C}$ expansion must fail. The lagrangian up to $C h O 4$ given by Gasser and Leutwyler [4] reads

$$
\begin{aligned}
\mathcal{L}^{G L}= & -\frac{f^{2}}{4}<\alpha_{\mu} \alpha^{\mu}>+\frac{f^{2} m^{2}}{4}<U+U^{\dagger}> \\
& +\frac{\ell_{1}^{r}}{4}<\alpha_{\mu} \alpha^{\mu}>^{2}+\frac{\ell_{2}^{r}}{4}<\alpha_{\mu} \alpha_{\nu}>^{2} \\
& -\frac{\ell_{4}^{r}}{4} m^{2}<\alpha_{\mu} \alpha^{\mu}\left(U+U^{\dagger}\right)>+\frac{\ell_{3}^{r}+\ell_{4}^{r}}{16} m^{4}<U+U^{\dagger}>^{2} \\
& +\ell_{5}^{r}<F_{\mu \nu}^{L} U F^{\mu \nu R} U^{\dagger}> \\
& +i \frac{\ell_{6}^{r}}{2}<F_{\mu \nu}^{L} D^{\mu} U D^{\nu} U^{\dagger}+F_{\mu \nu}^{R} D^{\mu} U^{\dagger} D^{\nu} U>
\end{aligned}
$$

where

$$
\begin{gathered}
\alpha_{\mu}=U^{\dagger} D_{\mu} U, \quad D_{\mu}=\partial_{\mu}+i\left[v_{\mu}, \cdot\right]-i\left\{a_{\mu}, \cdot\right\} \\
F_{\mu \nu}^{L, R}=\partial_{\mu}\left(v_{\nu} \pm a_{\nu}\right)-\partial_{\nu}\left(v_{\mu} \pm a_{\mu}\right)-i\left[\left(v_{\mu} \pm a_{\mu}\right),\left(v_{\nu} \pm a_{\nu}\right)\right]
\end{gathered}
$$

and $v_{\mu}\left(a_{\mu}\right)$ are external vector (axialvector) fields. Renormalized LECs and the respective $\gamma_{i}$ are given in table 2.3. Using these numbers (2.41) allows for a soliton solution; however, with the value of $\ell_{2}^{r}$ corresponding to a Skyrme parameter $e=7.24$ this soliton is unphysically small, compare table 1.1. Therefore one has to conclude that something is missing. To quantify 'something' and to get a better understanding of higher $C h O$ contributions at least at tree level, we investigate in the following a pionic $\mathrm{N} \ell \sigma$ model coupled to several other mesonic degrees of freedom, namely scalars, isovector vector and isoscalar vector mesons.

Neither is this list of resonances exhaustive nor is their inclusion into a chiral lagrangian unambiguous. Concerning these objections, at least the quality of the vector meson dominance (VMD) assumption on which our way to incorporate vector mesons is based can be judged using experimental values for $\mathrm{ChO} 4 \mathrm{LECs}$ as a reference. 


\subsubsection{Contributions from scalar mesons}

To estimate the effects of scalar mesons in a purely pseudoscalar model, we start from an $\mathrm{N} \ell \sigma$ model in which a scalar $\sigma$ meson has been introduced as a dilaton [19], 41] [42]. This model contains two parameters, the glueball condensate $C_{G} \simeq(300 \mathrm{MeV})^{4}$ [43] and the glueball mass $m_{G} \simeq 1200 \mathrm{MeV}$ leading to a scalar meson mass $m_{\sigma}=1209 \mathrm{MeV}$.

In order to obtain a purely pionic lagrangian up to $C h O$, we expand the scalar $\sigma$ field in powers of $1 / C_{G}$. Only the first two terms in this expansion are of relevance, higher ones would affect the result only at $\mathrm{ChO}>6$. Inserting the approximate $\sigma$ field back into $\mathcal{L}$, we arrive at

$$
\begin{aligned}
\mathcal{L} & =\frac{f_{\pi}^{2}}{2}\left\{\boldsymbol{\alpha}_{\mu} \boldsymbol{\alpha}^{\mu}-2 m_{\pi}^{2}(1-u)\right\} \\
& +\frac{f_{\pi}^{4}}{8 C_{G}}\left\{\boldsymbol{\alpha}_{\mu} \boldsymbol{\alpha}^{\mu}-3 m_{\pi}^{2}(1-u)\right\}^{2} \\
& +\frac{2}{m_{G}^{2} C_{G}} \partial_{\mu} \sigma^{(2)} \partial^{\mu} \sigma^{(2)}-\frac{4}{3 C_{G}^{2}}\left(\sigma^{(2)}\right)^{3}-\frac{3 f_{\pi}^{2} m_{\pi}^{2}}{2 C_{G}^{2}}\left(\sigma^{(2)}\right)^{2}(2-u)
\end{aligned}
$$

where we used

$$
\begin{aligned}
\sigma^{(2)} & =\frac{f_{\pi}^{2}}{4}\left\{\boldsymbol{\alpha}_{\mu} \boldsymbol{\alpha}^{\mu}-3 m_{\pi}^{2}(1-u)\right\} \\
\alpha_{\mu} & =i \boldsymbol{\tau} \cdot \boldsymbol{\alpha}_{\mu}, \quad u=\frac{1}{4}<U+U^{\dagger}>.
\end{aligned}
$$

Numerically, we have calculated the chiral angle $F$ from the original lagrangian 19 and inserted into (2.42) (note that for the present purpose (2.42) is understood not to contain external fields). The contributions to mass, $\pi N \sigma$-term and $g_{A}$ are then compared in table 2.1. Convergence in this case is excellent. (The Skyrmeterm, which is not affected from $\sigma$ meson exchange was omitted in this comparison.)

Likewise LECs could be read off and confronted with experimental values; however, we cannot expect agreement since we deliberately chose only to include the dilaton $\sigma$ meson; therefore we skip this here.

\subsubsection{Contributions from vector mesons}

Proceeding to calculate in analogous manner the effects of vector mesons, we use as a starting point the so called minimal model [13] [18] including symmetry breakers 44] and coupled to external sources. This is not the most general implementation of $\omega$ and $\varrho$ mesons as far as anomalous pieces of $\mathcal{L}$ 
Table 2.1: Comparison of exact and approximate scalar meson contributions. Each of the three intermediate columns contains the sum of approximate pieces up to and including the indicated $\mathrm{ChO}$.

\begin{tabular}{|c|c|c|c|c|}
\hline & $C h O=2$ & $C h O \leq 4$ & $C h O \leq 6$ & exact \\
\hline Mass $[\mathrm{MeV}]$ & 818.5 & 691.7 & 712.8 & 716.9 \\
\hline$\sigma_{\pi N}[\mathrm{MeV}]$ & 45.8 & 28.9 & 34.0 & 34.4 \\
\hline$g_{A}$ & .473 & .384 & .40 & .404 \\
\hline
\end{tabular}

are concerned [18] [45], but the differences can be shown [46] to manifest themselves only at $C h O \geq 8$.

Expansion of the $\varrho$ and $\omega$ fields in powers of the inverse vector meson mass and subsequent reinsertion into $\mathcal{L}$ yields (again up to $C h O$ 6)

$$
\begin{aligned}
\mathcal{L} & =\frac{f_{\pi}^{2}}{2}\left\{\boldsymbol{\alpha}_{\mu} \boldsymbol{\alpha}^{\mu}-2 m_{\pi}^{2}(1-u)\right\} \\
& -\frac{1}{8}<\varrho_{\mu \nu}^{(1)}>-\frac{m_{\pi}^{2}}{2 m_{\varrho}^{2}}<\left(D^{\mu} \varrho_{\mu \nu}^{(1)}-i g\left[V^{\mu}, \varrho_{\mu \nu}^{(1)}\right]\right) r^{\nu(1)}>-\frac{N_{C}}{2} v_{\mu}^{0} B^{\mu} \\
& -\frac{1}{4 m_{\varrho}^{2}}<\left(D^{\mu} \varrho_{\mu \nu}^{(1)}-i g\left[V^{\mu}, \varrho_{\mu \nu}^{(1)}\right]\right)^{2}>-\frac{m_{\pi}^{4}}{4 m_{\varrho}^{2}}<r_{\mu}^{(1)} r^{\mu(1)}> \\
& -\frac{1}{2} v_{\mu \nu}^{0} \omega^{\mu \nu(3)}+2\left(\frac{N_{C} g_{\omega}}{2 m_{\omega}}\right)^{2} B_{\mu} B^{\mu} .
\end{aligned}
$$

Here, the following abbreviations have been used:

$$
\begin{aligned}
\varrho_{\mu}^{(1)} & =V_{\mu}-\frac{1}{g} v_{\mu} \\
V_{\mu} & =\frac{i}{2 g}\left(\xi^{\dagger} D_{\mu} \xi-D_{\mu} \xi \xi^{\dagger}\right) \\
\omega_{\mu}^{(3)} & =-\frac{N_{C} g_{\omega}}{m_{\omega}^{2}} B_{\mu} \\
r_{\mu}^{(1)} & =i \frac{d}{g} \xi^{\dagger}\left(\left[U, \alpha_{\mu}\right]+\left[\alpha_{\mu}, U^{\dagger}\right]\right) \xi .
\end{aligned}
$$

$B_{\mu}$ is Skyrme's baryon current. The strength $d$ of the standard symmetry breaker for vector mesons is related to the $S U(3)$ meson mass differences $d=\left(m_{K^{*}}^{2}-m_{\varrho}^{2}\right) / 8\left(m_{K}^{2}-m_{\pi}^{2}\right)$. Moreover $v_{\mu}^{0}$ means an isoscalar vector field and the field strength tensors are defined in the usual way

$$
f_{\mu \nu}=\partial_{\mu} f_{\nu}-\partial_{\nu} f_{\mu}-i(g)\left[f_{\mu}, f_{\nu}\right]
$$


where the factor $g$ is in place if $f$ denotes a vector meson while it is absent in case of external fields. The coupling to external fields was considered since the local approximation can result in nonminimal couplings.

As an aside, one notes that $\varrho_{0}$ as well as $\omega_{i}$ are induced through the external vector field. Such components are important for certain properties (e.g. polarizabilities, see section 3.5) and have so far been missed in the calculations.

Without external fields, numbers are again computed by inserting the exact chiral angle obtained from the lagrangian according to [18] [44] for $g=2.9, g_{\omega}=2.2$ into (2.44). The comparison in table 2.2 then shows that the $C h O 6$ contribution due to the $\varrho$ is indeed much smaller (by a factor of 4) than the one of $\mathrm{ChO} 4$; nevertheless the approximation does not improve the exact value is, including $C h O 6$ underestimated by the same amount that it was overestimated using only $\mathrm{ChO} 4$. For the $\omega$ the first approximation differs from the exact result by a factor of 2 .

Table 2.2: Comparison of exact and approximate contributions to the soliton mass (in $M e V$ ) for $\varrho$ and $\omega$ mesons. The two intermediate columns contains the sum of approximate pieces up to and including the indicated $C h O$. They are further divided so as to display the contributions to symmetric $\left(\sim m_{\pi}^{0}\right)$ and symmetry breaking $\left(\sim m_{\pi}^{(2 n)}, \quad n>0\right)$ pieces individually. Note that, although the original Lagrangian contains terms with $n=1$ at most, its approximate may comprise terms of arbitrary $n$.

\begin{tabular}{|c|cc|ccc|cc|}
\hline & \multicolumn{2}{|c|}{$C h O \leq 4$} & \multicolumn{3}{c|}{$C h O \leq 6$} & \multicolumn{2}{c|}{ exact } \\
& $m_{\pi}^{0}$ & $m_{\pi}^{2}$ & $m_{\pi}^{0}$ & $m_{\pi}^{2}$ & $m_{\pi}^{4}$ & $m_{\pi}^{0}$ & $m_{\pi}^{2}$ \\
\hline$\varrho$ & 494.6 & - & 377.6 & 5.9 & -.36 & 439.0 & 5.66 \\
\hline$\omega$ & - & - & 475.3 & - & - & 235.85 & - \\
\hline
\end{tabular}

Since $\omega$ can be expressed exactly in terms of the baryon source, it is easy to go beyond this approximation. We have

$$
\begin{aligned}
\omega_{0}(r) & =-N_{C} g_{\omega} \int d^{3} r^{\prime} \int \frac{d^{3} q}{(2 \pi)^{3}} \frac{e^{i} \boldsymbol{q}\left(\boldsymbol{r}-\boldsymbol{r}^{\prime}\right)}{\boldsymbol{q}^{2}+m_{\omega}^{2}} B_{0}\left(r^{\prime}\right) \\
& =-\frac{N_{C} g_{\omega}}{m_{\omega}^{2}} \sum_{n}\left(\frac{\partial_{r}^{2}}{m_{\omega}^{2}}\right)^{n} B_{0}(r)
\end{aligned}
$$

for the (only nonzero) static field $\omega_{0}$. If $\partial_{r}^{2} B_{0}$ and $m_{\omega}^{2}$ are of the same magnitude, the approximation will not converge. This indeed seems to be the 
case.

From the above reasoning, it is obvious that the $C h O 4$ lagrangian accounts for all effects from scalar mesons and to a large extent for $\varrho$ mesons, too. This last statement is also obvious from the comparison of VMD predicted versus experimentally known $L E C s$, table 2.3. (The $\gamma_{i}$ used in this table are taken from [4, LECs from 47]).

Table 2.3: LECs at scales of $\mu=m_{\eta}$ and $\mu=m_{\varrho}$, respectively, compared to VMD prediction.

\begin{tabular}{|c|c|c|c|c|}
\hline & $\gamma_{i}$ & $\ell_{i}^{r}\left(\mu=m_{\eta}\right) \cdot 10^{3}$ & $\ell_{i}^{r}\left(\mu=m_{\varrho}\right) \cdot 10^{3}$ & $\mathrm{VMD}$ \\
\hline$\ell_{1}^{r}$ & $1 / 3$ & $-3.94 \pm 1.3$ & $-4.65 \pm 1.3$ & - \\
\hline$\ell_{2}^{r}$ & $2 / 3$ & $6.35 \pm 1.5$ & $4.92 \pm 1.5$ & $1 /\left(16 g^{2}\right)=7.3$ \\
\hline$\ell_{3}^{r}$ & $-1 / 2$ & $-0.22 \pm 3.8$ & $-0.85 \pm 3.8$ & - \\
\hline$\ell_{4}^{r}$ & 2 & $9.74 \pm 5.7$ & $5.46 \pm 5.7$ & - \\
\hline$\ell_{5}^{r}$ & $-1 / 6$ & $-5.88 \pm 0.7$ & $-5.22 \pm 0.7$ & $-1 /\left(16 g^{2}\right)=-7.3$ \\
\hline$\ell_{6}^{r}$ & $-1 / 3$ & $-14.5 \pm 1.2$ & $-13.8 \pm 1.2$ & $-1 /\left(8 g^{2}\right)=-14.6$ \\
\hline
\end{tabular}

Figure 2.2: Comparison of energy densities of purely pionic models with the three parameter sets $A, B$ and $C$ (solid lines) and the exact density from the VMD lagrangian with $g=$ 2.9, $g_{\omega}=2.2$ (dashed line). 
What is missing in (2.41) essentially is due to the $\omega$ meson. How then can we account for this? To answer, we compared the energy density of a VMD minimal model with $g=2.9 g_{\omega}=2.2$ [18] to its local approximate ((2.44) without symmetry breakers and 6th order contributions from $\varrho$ ). The chiral angle was in this case computed from the approximate lagrangian, and the result is plotted in fig. 2.2. (For a converging local approximation, this computational difference shouldn't matter; however, since we suspected lack of convergence for $\omega$, and since we would use a chiral angle from a purely pionic model in our calculations of 1-loop corrections, we had to do the comparison in this way.) From fig. 2.2 it is obvious that much better agreement may be obtained by calculating the chiral angle from an approximate lagrangian with parameters reduced to $g_{\omega} \simeq 1.0$ and $e=4.5$ : the missing higher chiral orders renormalize the original parameters to effective values. Eventually, a computationally convenient model with $e=4.25$ and $g_{\omega}=0$ is not worse than the original $e=5.8, \quad g_{\omega}=2.2$ combination.

These considerations result in the lagrangian

$$
\begin{aligned}
\mathcal{L} / f_{\pi}^{2} & =\frac{1}{2} \boldsymbol{\alpha}_{\mu} \boldsymbol{\alpha}^{\mu}+m_{\pi}^{2}(u-1) \\
& -\frac{1}{4} c_{4}^{a}\left[\left(\boldsymbol{\alpha}_{\mu} \boldsymbol{\alpha}^{\mu}\right)^{2}-\left(\boldsymbol{\alpha}_{\mu} \boldsymbol{\alpha}_{\nu}\right)^{2}\right]+\frac{1}{2} c_{4}^{s}\left(\boldsymbol{\alpha}_{\mu} \boldsymbol{\alpha}^{\mu}\right)^{2} \\
& +c_{4}^{k}\left(\boldsymbol{\alpha}_{\mu} \boldsymbol{\alpha}^{\mu}\right)(u-1)+c_{4}^{m}(u-1)^{2} \\
& +c_{4}^{e}<F_{\mu \nu}^{L} U F^{\mu \nu R} U^{\dagger}>+i \frac{c_{4}^{f}}{2}<F_{\mu \nu}^{L} D^{\mu} U D^{\nu} U^{\dagger}+F_{\mu \nu}^{R} D^{\mu} U^{\dagger} D^{\nu} U> \\
& -\frac{N_{C}}{2 f_{\pi}^{2}} v_{\mu}^{0} B^{\mu}-2 \pi^{4} c_{6} B_{\mu} B^{\mu}-\frac{1}{4 m_{\omega}^{2}} v_{\mu \nu} B^{\mu \nu} \\
c_{4}^{a} & =\frac{4 \ell_{2}^{r}}{f_{\pi}^{2}}=\frac{1}{f_{\pi}^{2} e^{2}} \quad c_{4}^{s}=2 \frac{\ell_{1}^{r}+\ell_{2}^{r}}{f_{\pi}^{2}} \quad c_{4}^{k}=m^{2} \frac{\ell_{4}^{r}}{f_{\pi}^{2}} \quad c_{4}^{m}=m^{4} \frac{\ell_{3}^{r}+\ell_{4}^{r}}{f_{\pi}^{2}} \\
c_{4}^{e} & =\frac{\ell_{5}^{r}}{f_{\pi}^{2}}=-\frac{c_{4}^{a}}{4} \quad c_{4}^{f}=\frac{\ell_{6}^{r}}{f_{\pi}^{2}}=-\frac{c_{4}^{a}}{8} \quad c_{6}=\frac{1}{4 \pi^{4} f_{\pi}^{2}}\left(\frac{N_{C} g_{\omega}}{m_{\omega}}\right)^{2} .
\end{aligned}
$$

Note that only the photon $v_{\mu}$ can couple nonminimally to the baryon current. For comparison purposes, in the following evaluations, we therefore use (2.47) with the parameter combinations mentioned above, which will henceforth be referred to as models

$$
\begin{aligned}
& A \equiv\left(e=4.25, g_{\omega}=0\right) \\
& B \equiv\left(e=4.5, g_{\omega}=1.0\right) \\
& C \equiv\left(e=5.8, g_{\omega}=2.2\right) .
\end{aligned}
$$

These parameters are used to determine $c_{4}^{a}$ and $c_{6}$ as well as the constants $c_{4}^{e}, c_{4}^{f}$ regulating the contributions of nonminimal terms, whereas $c_{4}^{k}$ and $c_{4}^{m}$, 
both scalar meson induced, are kept at their experimental values for $\mu=m_{\varrho}$. One notes that the constant in front of the nonminimal term produced by the $\omega$ depends solely on the vector meson mass and is therefore not affected by the use of 'effective' coupling constants. Unless otherwise stated, $c_{4}^{s}$ is set to zero in accordance with experiment. Finally, the finite renormalization of $f \rightarrow f_{\pi}, m \rightarrow m_{\pi}$ is expressed through the formulae

$$
f_{\pi}^{2}=f^{2}+2 \ell_{4}^{r} m^{2}, \quad f_{\pi}^{2} m_{\pi}^{2}=f^{2} m^{2}+2\left(\ell_{3}^{r}+\ell_{4}^{r}\right) m^{4} .
$$

To summarise, we have investigated the local approximations on models containing explicit scalar and vector meson degrees of freedom in order to better understand the role of higher $(\mathrm{ChO}>4)$ chiral orders in the chiral lagrangian, which, in contrast to standard ChPT cannot be dismissed out of hand in the soliton sector. We concluded that terms of higher chiral orders due to scalar mesons and isovector vector mesons are essentially unimportant, but that there is no a priori justification to drop those induced by the isoscalar vector meson. Since we could not take those terms into account properly, we used, as an alternate means to handle this problem, 'effective' coupling constants in the terms generated by vector mesons These coupling constants differ from the ones in the original vector meson lagrangian; comparison of exact and approximate energy densities led to parameter combination $B$, whereas $A$ and $C$ would serve as reference sets, with $C$ using paramters unchanged relative to the exact lagrangian and $A$ being particularly convenient to work with.

\subsection{One-loop corrections for lagrangian with explicit vector and scalar mesons}

Although we use a purely pseudoscalar lagrangian for the calculation of 1loop corrections in this report, we briefly discuss the inclusion of other meson species like vector and scalar mesons. Such models [13] [15]-[19] have been quite sucessful in some respects (high-energy behaviour of pion-nucleon phaseshifts, formfactors).

The most general lagrangian of this kind would be of the form

$$
\mathcal{L}_{x}=\mathcal{L}_{\text {eff }}(U)+\sum_{x} \mathcal{L}_{\text {int }}(U, x)+\sum_{x} \mathcal{L}_{\text {res }}(x)
$$

where $x=\varrho, \omega, \sigma \ldots$ generically denotes all possible resonance degrees of freedom and $\mathcal{L}_{\text {eff }}$ is of the form (2.1). It is clear that such an object would be even less manageable than the purely pseudoscalar lagrangian and has to 
be accompanied by some simplifying assumptions. The first one is obviously the restriction to low lying resonances, i.e. vector and (two kinds of) scalar mesons. The second one uses the fact that upon expanding the resonances in terms of pseudoscalars, $\mathcal{L}_{x}$ itself is of the form (2.1). Calculating 1-loop processes, we have $\ell_{i}^{(N)} \rightarrow \ell_{i}^{(N) r}(\mu)$ as previously, but for $N \geq 4, \ell_{i}^{(N) r}$ may be decomposed into a resonant and a direct part

$$
\ell_{i}^{(N) r}(\mu)=\ell_{i r e s}^{(N)}+\ell_{i \text { direct }}^{(N) r}(\mu)
$$

where $\ell_{i \text { res }}^{(N)}$ depends solely on resonance mass and coupling constant, but carries no scale dependence. The assumption then is that there exists a scale such that $\ell_{i \text { direct }}^{N) r}(\mu) \simeq 0$, which is an alternative way to spell the concept of vector (and scalar) meson dominance. This postulate is considerably weaker than its analogue in the purely pseudoscalar case, namely to assume the existence of a scale where $\ell_{i}^{(N)}(\mu) \simeq 0$ for all higher chiral order terms which cannot be accomodated within our formalism. This constitutes the main conceptual advantage of a calculation involving resonances explicitly. The total soliton mass in tree +1 -loop (2.28) generalizes to

$$
M(\mu)=M_{0}+\sum_{x} E_{c a s}^{x}(\mu)
$$

The classical soliton mass $M_{0}$ depends on parameters introduced by the additional mesons, e.g. $g_{\varrho}, g_{\omega} \ldots$. All mesons contribute to the Casimir energy via their phaseshift $\delta^{x}(p)$ which is a sum over all channel eigen phases. The number of channels may be considerable, e.g. 9 channels for the $\varrho$-meson, which makes an accurate determination of the phaseshifts technically difficult. A nice feature is however that in the high momentum region these phaseshifts behave well, i.e. in the individual partial wave they tend to zero in contrast to those of purely pseudoscalar models. Consequently the determination of the asymptotical constants

$$
\delta^{x}(p) \stackrel{p \rightarrow \infty}{\longrightarrow} a_{1}^{x} p+\frac{a_{2}^{x}}{p}+\mathcal{O}\left(p^{-3}\right)
$$

should be less critical compared to the pure pseudoscalar case which involves an additional term $a_{0} p^{3}$ (compare eq. (2.22)). With these preparations the contributions of the individual meson species $x$ to the Casimir energy may be evaluated with the phaseshift formula (2.26)

$$
\begin{aligned}
E_{c a s}(\mu)= & \frac{1}{2 \pi}\left(\int_{0}^{\infty} \frac{d p}{\sqrt{p^{2}+m_{x}^{2}}}\left[-p\left(\delta^{x}(p)-a_{1}^{x} p\right)+a_{2}^{x}\right]-m_{x} \delta^{x}(0)\right. \\
& \left.-\frac{m_{x}^{2} a_{1}^{x}}{4} \ln \frac{m_{x}^{2}}{\mu^{2}}+\frac{a_{2}^{x}}{2}\left(1+\ln \frac{m_{x}^{2}}{\mu^{2}}\right)\right) .
\end{aligned}
$$


Because the meson mass $m_{x}$ is smallest for pions it is expected that their contribution dominates the Casimir energy, the contributions of other mesons being suppressed by their larger mass.

There remains the question concerning the chiral scale $\mu$. For a $\pi \varrho \omega$ model which in addition includes both sorts of scalar mesons the LECs in ChO 4 compare well 47 with those obtained in $C h P T$ at $\mu \simeq m_{\varrho}$ (table 2.3). Therefore, such a choice should be quite reasonable. Anyhow the Casimir energy will not react very sensitively on small changes in the chiral scale because it enters only logarithmically.

Below, we list merits and problems of using lagrangians with explicit vector- and scalar-mesons.

Advantages:

- The LECs of higher chiral order terms are fixed form the assumption of vector (scalar) meson dominance and not simply neglected as in the pseudoscalar case.

- the high momentum phaseshifts behave well

- all parameters are fixed in the meson sector, these models possess no free parameters.

Disadvantages:

- the inclusion of additional mesons is not unique as far as the anomaly is concerned

- next higher resonances (e.g. axial vectors etc.) are treated only approximately

- the tree calculations have to be updated (induced components, e.g. polarizabilities)

- a huge coupled channel problem has to be solved with sufficiently high precision.

In the end we decided to use a pseudoscalar lagrangian only. We are confident that, concerning 1-loop corrections, the results for a lagrangian with explicit $\varrho$ and $\omega$-mesons would come close to those of our model $B$. In particular the problems with the axial quantities discussed in section 3.3 are not expected to be cured by the introduction of vector mesons. 


\section{Chapter 3}

\section{Baryon properties}

In the following sections, we are going to display the specifics of the way to calculate various baryon properties of interest.

For the presentation, we shall adopt the following pattern: For each quantitiy under investigation, we will first give some basic definitions and experimental findings. In parallel, we explain the choice of external field so as to admit a hedgehog solution. We then show a recalculation of the tree level value comprising all relevant terms of the lagrangian (2.47). In case of the electric polarizability, doubts had been voiced regarding the correct way to calculate it; we will discuss this issue and conclude that the standard way is indeed correct.

Proceeding to the 1-loop calculation, we derive the e.o.m for the fluctuations and discuss problems which arise in several instances. They concern the proper meaning of vacuum subtraction ( $\sigma$-term, e.m. formfactors) and the treatment of apparently non local terms (electric polarizability).

For a more convenient comparison, numerical results will then be discussed in a separate section. As always, there is an exception from the rule; in the present case this involves the axial coupling constant, where we give results immediately since we are forced to investigate in detail the implications of current algebra for the $1 / N_{C}$ expansion of this quantity.

The full list of calculated quantities involves

- the Baryon mass (section 3.1),

- the $\pi N \sigma$ term and scalar radius (section 3.2),

- the axial coupling constant $g_{A}$ and the corresponding axial radius (section 3.3), 
- the electromagnetic formfactors (more specifically, the isovector magnetic moment and corresponding radius and the isoscalar electric radius) (section 3.4),

- the electric polarizability of the nucleon (section 3.5).

- the electromagnetic properties of the $\delta$ isobar (section 3.6).

With respect to the polarizability, we opined that its neutron proton split deserves a derivation, which has therefore been included in section 3.5.3 .

Although the list of calculated quantities is fairly long, it is by no means exhaustive, since we are limited to quantities where the correction is brought about by 'adiabatic loops', i.e., loop graphs, in which the pion can be treated in adiabatic approximation. A calculation involving nonadiabatic fluctuations would also have to deal with (adiabatic) 2-loop graphs which appear at the same level in the $1 / N_{C}$ counting. Such a project is well beyond our present abilities.

\subsection{Baryon mass}

\subsubsection{Tree approximation}

The classical soliton mass is obtained by inserting the hedgehog ansatz into the lagrangian (2.47). For convenience, we will give this quantity in terms of the longitudinal and transversal metric appearing in (2.17),

$$
n_{a b}^{2}=b_{L}(r) \hat{r}_{a} \hat{r}_{b}+b_{T}(r)\left(\delta_{a b}-\hat{r}_{a} \hat{r}_{b}\right),
$$

which allows to express the mass and most of the other quantities in the subsequent sections in a very compact way without reference to the specific terms contained in the lagrangian. For our choice, these functions are given by

$$
\begin{aligned}
& b_{L}=1+2 c_{4}^{a} \frac{s^{2}}{r^{2}}-2 c_{4}^{s}\left(F^{\prime 2}+\frac{2 s^{2}}{r^{2}}\right)-2 c_{4}^{k}(1-c)+c_{6} \frac{s^{4}}{r^{4}} \\
& b_{T}=1+c_{4}^{a}\left(F^{\prime 2}+\frac{s^{2}}{r^{2}}\right)-2 c_{4}^{s}\left(F^{\prime 2}+\frac{2 s^{2}}{r^{2}}\right)-2 c_{4}^{k}(1-c)+c_{6} \frac{F^{\prime 2} s^{2}}{r^{2}}
\end{aligned}
$$

where abbreviations $s=\sin (F), c=\cos (F)$ were used.

Variation of the classical soliton mass

$$
M_{0}=\frac{f_{\pi}^{2}}{3} \int d^{3} r\left[F^{\prime 2} b_{L}+\frac{2 s^{2}}{r^{2}} b_{T}\right]
$$


with respect to the chiral angle yields the stability condition

$$
\begin{aligned}
\frac{1}{r^{2}}\left(r^{2} F^{\prime} b_{L}\right)^{\prime}= & \frac{2 s c}{r^{2}} b_{T}+m_{\pi}^{2} s \\
& -c_{4}^{k} s\left(F^{\prime 2}+\frac{2 s^{2}}{r^{2}}\right)-2 c_{4}^{m} s(1-c),
\end{aligned}
$$

an ordinary nonlinear differential equation, which has to be solved numerically subject to the boundary conditions $F(0)=\pi, F(\infty)=0$ which guarantee a solution carrying baryon number $B=1$. Clearly the soliton mass is of $\mathcal{O}\left(N_{C}\right)$.

It was the fact that the classical mass (3.3) always came out much too large in soliton models with realistic parameters which finally led to the investigation of loop corrections [21] [31]-[36] which in the end turned out to considerably lower [34] [35] [36] the numerical values for this quantity.

Similar to the soliton mass, we obtain for the moment of inertia

$$
\Theta=\frac{2 f_{\pi}^{2}}{3} \int d^{3} r s^{2} b_{T}
$$

which according to (2.11) determines the nucleon- $\Delta$ split

$$
\Delta=\frac{3}{2 \Theta}
$$

a nonadiabatic quantity of $\mathcal{O}\left(N_{C}^{-1}\right)$ related to the angular rotation. As already mentioned, we do not report about loop corrections to nonadiabatic quantities although they are by no means less important for the simple reason that such calculations would become tremendously complicated.

Another nonadiabatic quantity related to the mass is the neutron proton split which vanishes for the lagrangian (2.47) because there exists no term that distinguishes between states of different isospin 3-component. In fact, it can be shown that such a terms must not appear before $C h O 8$ 46. The $\mathrm{ChO} 8$ term is isolated by local approximation from the standard symmetry breaker for vector mesons and is related to $\varrho \omega$ mixing [44, 48,

$$
\begin{aligned}
L^{\varrho \omega} & =\frac{i g_{\omega} N_{C} \mu_{\varrho \omega}^{2}}{16 g_{\varrho} m_{\varrho}^{2} m_{\omega}^{2}} \int d^{3} r \partial^{\nu} B^{\mu}<\left(U \tau_{3}+\tau_{3} U^{\dagger}\right)\left[\alpha_{\mu}, \alpha_{\nu}\right]> \\
& \stackrel{\text { tree }}{=}\left(\Theta M_{n p}\right) D_{3 a} \Omega_{a}^{R} .
\end{aligned}
$$

Here, $B^{\mu}$ is again the familiar baryon current. With experimental values for $g_{\omega}=3.3, g_{\varrho}=2.8, m_{\omega}=782 \mathrm{MeV}, m_{\varrho}=768 \mathrm{MeV}$ and for the $\varrho \omega$ mixing 
parameter $\mu_{\varrho \omega}^{2}=(-4.5 \pm 0.6) \cdot 10^{-3} \mathrm{GeV}^{2}$ [49] [50] quite a reasonable result is obtained for the $n p$ split

$$
M_{n p}=\frac{g_{\omega} N_{C} \mu_{\varrho \omega}^{2}}{3 \Theta g_{\varrho} m_{\varrho}^{2} m_{\omega}^{2}} \int d^{3} r B^{0}\left(\frac{2 F^{\prime} s}{r}+\frac{s^{2} c}{r^{2}}\right), \quad B^{0}=-\frac{F^{\prime} s^{2}}{2 \pi^{2} r^{2}},
$$

compare table 4.5 .

\subsubsection{Loop corrections and scale dependence of the soliton mass}

For the 1 loop corrections adiabatic fluctuations around the soliton background according to the parametrization (2.14) are introduced and conveniently decomposed into longitudinal and transversal components

$$
\boldsymbol{\eta}=\eta_{L} \hat{\boldsymbol{r}}+\boldsymbol{\eta}_{T}
$$

From the lagrangian expanded to quadratic order in the fluctuations

$$
\begin{aligned}
L \stackrel{\eta^{2}}{=} & \frac{1}{2} \int d^{3} r\left(\dot{\boldsymbol{\eta}}^{2}-\partial_{i} \boldsymbol{\eta} \partial_{i} \boldsymbol{\eta}+\frac{4 c}{r} \eta_{L} \boldsymbol{\nabla} \boldsymbol{\eta}_{T}\right. \\
& \left.+\frac{2\left(c^{2}-s^{2}\right)}{r^{2}} \eta_{L}^{2}-\left(F^{2}+\frac{2 s^{2}}{r^{2}}\right) \boldsymbol{\eta}_{T}^{2}+\cdots\right) \\
= & \frac{1}{2} \int d^{3} r\left(\dot{\eta}_{a} n_{a b}^{2} \dot{\eta}_{b}+\eta_{a} h_{a b}^{2} \eta_{b}\right)
\end{aligned}
$$

the operators $n_{a b}^{2}$ and $h_{a b}^{2}$ which determine the e.o.m (2.18) may be read off. For simplicity, we give only the terms generated by the $\mathrm{N} \ell \sigma$ model explictly.

Technically, it is convenient to write the fluctuations in terms of vector spherical harmonics

$$
\boldsymbol{\eta}(\boldsymbol{r}, t)=\sum_{L \ell} f_{L \ell}(r) \boldsymbol{Y}_{L \ell M}(\hat{\boldsymbol{r}}) e^{-i \omega t}
$$

which decouple the e.o.m into electric $\ell=L \pm 1$ and magnetic $\ell=L$ modes. This procedure is standard and the full differential equations for the radial functions $f_{L \ell}(r)$ are given elsewhere [36] [39] [51]. The challenge is now to solve these coupled channel equations for the phaseshifts with high enough accuracy which implies large phonon spins $\left(L_{\max } \simeq 100\right)$ and large momenta $\left(p_{\max } \simeq 25 m_{\pi}\right)$ such that the sum

$$
\delta(p)=\sum_{L c}(2 L+1) \delta_{L}^{c}(p)
$$


converges and the asymptotic behaviour can be reliably extracted. For this purpose we use the so called variable phase method [52] which allows to solve for all desired phonon spins simultaneously. The result is plotted in fig. 3.1 where the asymptotic behaviour has already been subtracted (a similar picture appears in [36] ). Because of the zero modes and in accordance with Levinson's theorem this subtracted phaseshift starts close to $6 \pi$ and falls off rapidly on a momentum scale of $2 m_{\pi}$. It is essentially this function

Figure 3.1: Scattering phaseshift from model $A$ with asymptotic behaviour subtracted.

which enters the expression (2.26) for the Casimir energy. The asymptotical constants $a_{0}, a_{1}, a_{2}$ are known analytically for the $\mathrm{N} \ell \sigma$ model which fact served as a test for the program. Numerical values for the full lagrangian are close to those given by Moussallam [36].

Next, we are going to investigate the dependence of the soliton mass in 1-loop approximation 2.26, 2.28) on the chiral scale $\mu$. In close analogy to the $1+1$ dimensional kink 30] we may expect scale independence only to zeroth order in the large expansion parameter of the theory, which in our case is $N_{C}$ (an $\mathcal{O}\left(N_{C}^{-1}\right)$ scale dependence must in principle be compensated by 2-loop contributions).

In order to accomplish this task we have to choose the scale, say $\mu=m_{\varrho}$, such that the lagrangian is dominantly $\mathcal{O}\left(N_{C}\right)$ and then we may look at the scale dependence of $M(\mu)$ in the vicinity of $\mu \simeq m_{\varrho}$. Because we do not know 
the $L E C s$ of $C h O \geq 6$ and their scale dependence, we use the pure 4 th order lagrangian $A$.

Before we discuss the numerical results, we will show analytically that the mass is indeed scale independent to $\mathcal{O}\left(N_{C}^{0}\right)$ in the vicinity of $\mu \simeq m_{\varrho}$ provided all $\mathrm{ChOs}$ higher than those already contained in the starting lagrangian are negligible.

For this purpose we notice that the LECs of $C h O 4$ scale according to

$$
\ell_{i}^{r}(\mu)=\ell_{i}^{r}\left(m_{\varrho}\right)-\frac{\gamma_{i}}{32 \pi^{2}} \ln \left(\frac{\mu^{2}}{m_{\varrho}^{2}}\right), \quad \ell_{i}^{r}\left(m_{\varrho}\right)=\mathcal{O}\left(N_{C}\right) .
$$

In the vicinity of $\mu \simeq m_{\varrho}$ the soliton mass (2.28) behaves like

$$
\begin{aligned}
M_{0}\left(\ell_{i}^{r}(\mu)\right) \stackrel{\mu \simeq m_{\varrho}}{\simeq} M_{0}\left(\ell_{i}^{r}\left(m_{\varrho}\right)\right)+\frac{1}{32 \pi^{2}} \ln \left(\frac{\mu^{2}}{m_{\varrho}^{2}}\right) \sum_{i=1}^{4} \gamma_{i} \int d^{3} r \mathcal{L}_{i} \\
=M_{0}\left(\ell_{i}^{r}\left(m_{\varrho}\right)\right)+\frac{1}{2 \pi}\left[-\frac{m_{\pi}^{2} a_{1}^{(2)}}{4}+\frac{a_{2}^{(4)}}{2}\right] \ln \left(\frac{\mu^{2}}{m_{\varrho}^{2}}\right) .
\end{aligned}
$$

Because the stability condition for the $N_{C}$ soliton is calculated from $M_{0}\left(\ell_{i}^{r}\left(m_{\varrho}\right)\right)$ there is no contribution from the $\mu$ dependence of the chiral angle. The low energy constants $\ell_{i}^{r}(\mu)$ enter the e.o.m for the fluctuations and consequently also the phaseshifts $\delta(p)$ as well as the asymptotical constants $a_{0}, a_{1}, a_{2}$ as ratios $\ell_{i}^{r}(\mu) / f^{2}$ which are all of $\mathcal{O}\left(N_{C}^{0}\right)$. From (3.13) it is then immediately clear that the $\mu$ dependence in all these quantities, $E_{c a s}^{0}$ included, does not appear until $\mathcal{O}\left(N_{C}^{-1}\right)$. In $C h O 4$ the remaining terms in (3.14) just compensate for the scale dependence of the counter terms in (2.26, 2.28) $\left(a_{0}\right.$ is at least $\left.C h O 2\right)$ which is then scale independent to $\mathcal{O}\left(N_{C}^{0}\right)$.

However the constants $m^{4} a_{0}, m^{2} a_{1}, a_{2}$ contain all chiral orders, and the higher orders $(C h O \geq 6)$ are not compensated for. Therefore, the expression (2.26, 2.28) cannot be strictly scale invariant even at $\mathcal{O}\left(N_{C}^{0}\right)$. Invariance could only be restored if higher $C h O$ terms in the lagrangian were switched on upon leaving $\mu=m_{\varrho}$ where they were assumed to be zero. This implies that the scale dependence calculated numerically does not only comprise of small $\mathcal{O}\left(N_{C}^{-1}\right)$ effects but also measures the magnitude of higher $C h O$ terms not accounted for through the usage of an effective Skyrme parameter. All the more it comes as a surprise that the soliton mass in tree +1 loop depicted in fig. 3.2 (a) (solid line) turns out to be almost scale independent over a very large region of $\mu$. The scale dependence of the tree contribution (dashed line) is nicely compensated for by that of the 1-loop piece. At small scales $\mu \leq$ $550 \mathrm{MeV}$ the onset of scale dependence is rapid till the soliton is destroyed at $\mu \simeq 420 \mathrm{MeV}$ by the increasing $\mathrm{ChO} 4$ symmetric term $\left(c_{4}^{s}=0.017 m_{\pi}^{-2}\right.$, [53]). 
For comparison, we also evaluated a model with $e=7.24$ at $\mu=770 \mathrm{MeV}$, this choice corresponds to the value implied by the numerical size of the $L E C$ s at scale $\mu=m_{\varrho}$ (table 2.3$)$.

(a)

(b)

Figure 3.2: Scale dependence of the soliton mass for models which at scale $\mu=770 \mathrm{MeV}$ correspond to $(a) e=4.25\left(g_{\omega}=0.0\right)$ and $(b)$ $e=7.24\left(g_{\omega}=0.0\right)$. Dashed lines: tree values, solid lines: tree +1 -loop. 
Any notion of scale independence ceases to exist in this case (fig. 3.2 (b) ) which strongly supports the conjecture that the usage of effective parameters might emulate missing higher chiral order terms.

\subsection{Scalar properties}

The scalar field $\varsigma$ which couples to the quark mass matrix is introduced into the $S U(2)$ lagrangian (2.47) by the replacement $m^{2} \rightarrow m^{2}(1+\varsigma)$

$$
L(\varepsilon)=L-\varepsilon f_{\pi}^{2} \int d^{3} r \varsigma\left[m_{\pi}^{2}(1-u)+2 c_{4}^{m} u(1-u)-c_{4}^{k} \boldsymbol{\alpha}_{\mu} \boldsymbol{\alpha}^{\mu} u\right] .
$$

The special choice

$$
\varsigma=i_{0}(\sqrt{t} r)=\frac{\sinh (\sqrt{t} r)}{\sqrt{t} r}, \quad t=\left(p-p^{\prime}\right)^{2}
$$

where $p$ and $p^{\prime}$ denote the nucleon four momenta leads to

$$
M(\varepsilon)=M+\varepsilon \sigma(t), \quad \sigma(t)=\left.\frac{\partial M(\varepsilon)}{\partial \varepsilon}\right|_{\varepsilon=0},
$$

the scalar formfactor $\sigma(t)$ in the time like region.

According to the $\sigma$-term update [27] the $\sigma$-term should lie around $\sigma=$ $\sigma(0) \simeq 45 \mathrm{MeV}$. The interesting quantity for the extrapolation of the $\sigma$-term from the Cheng-Dashen point is $\sigma\left(2 m_{\pi}^{2}\right)-\sigma(0)$ which is estimated in [54] to be $15.2 \pm 0.4 \mathrm{MeV}$. This value is connected with an extraordinarily large scalar radius $<r^{2}>_{\sigma}=6 \sigma^{\prime}(t) / \sigma=1.6 \mathrm{fm}^{2}$.

\subsubsection{Tree approximation}

The scalar formfactor in tree approximation is immediately obtained from

$$
\sigma(t) \stackrel{\text { tree }}{=} f_{\pi}^{2} \int d^{3} r i_{0}(\sqrt{t} r)\left[m_{\pi}^{2}(1-c)+2 c_{4}^{m} c(1-c)+c_{4}^{k}\left(F^{\prime 2}+2 \frac{s^{2}}{r^{2}}\right) c\right]
$$

There are eventually relativistic corrections [57] which however neither affect the $\sigma$-term nor the scalar radius

$$
\begin{array}{r}
\sigma \stackrel{\text { tree }}{=} f_{\pi}^{2} \int d^{3} r\left[m_{\pi}^{2}(1-c)+2 c_{4}^{m} c(1-c)+c_{4}^{k}\left(F^{\prime 2}+2 \frac{s^{2}}{r^{2}}\right) c\right] \\
<r^{2}>_{\sigma} \stackrel{\text { tree }}{=} \frac{f_{\pi}^{2}}{\sigma} \int d^{3} r r^{2}\left[m_{\pi}^{2}(1-c)+2 c_{4}^{m} c(1-c)+c_{4}^{k}\left(F^{\prime 2}+2 \frac{s^{2}}{r^{2}}\right) c\right] .
\end{array}
$$


The main contribution to the $\sigma$-term is supplied by the familiar pion mass term of $\mathrm{ChO} 2$ (first term), the $\mathrm{ChO} 4$ terms contribute only about $10 \mathrm{MeV}$.

In the chiral limit $m_{\pi} \rightarrow 0$ the scalar square radius $\left\langle r^{2}\right\rangle_{\sigma}$ diverges as is noticed when the asymptotic behaviour of the chiral angle

$$
F \stackrel{r \rightarrow \infty}{\rightarrow} \frac{3 g_{A}}{8 \pi f_{\pi}^{2}} \frac{1+m_{\pi} r}{r^{2}} e^{-m_{\pi} r}
$$

is inserted into (3.19) . In particular, we obtain in this limit

$$
6 \sigma^{\prime}(0)=<r^{2}>_{\sigma} \sigma \stackrel{m_{\pi} \rightarrow 0}{=} \frac{f_{\pi}^{2} m_{\pi}^{2}}{2} \int d^{3} r r^{2} F^{2} \stackrel{m_{\pi} \rightarrow 0}{=} \frac{45 g_{A}^{2} m_{\pi}}{128 \pi f_{\pi}^{2}},
$$

the standard result of $H B C h P T$ with external nucleons [6] [55]. This is due

to the $\Delta$ states degenerate with the nucleon in soliton models at leading order $N_{C}$ [56].

In general, soliton models, provided realistic parameters are used, overestimate the $\sigma$-term and underestimate the scalar radius in tree approximation, the latter deficiency being caused by the former. Eventually, loop corrections are able to cure both shortcomings.

\subsubsection{Loop corrections to the scalar formfactor}

Here we will discuss the calculation of the Casimir energy in the presence of the external scalar field (3.16). The hedgehog remains solution and the stability condition obtained by variation of the tree expression (3.17) with respect to the chiral angle $F$ picks up contributions from $\sigma(t)$ (3.18) .

From the terms quadratic in the fluctuations

$$
\begin{aligned}
L(\varepsilon) & \stackrel{\eta^{2}}{=} L-\frac{\varepsilon}{2} \int d^{3} r i_{0}(\sqrt{t} r)\left(-2 c_{4}^{k} c \dot{\boldsymbol{\eta}}^{2}+2 c_{4}^{k} c\left(\partial_{i} \boldsymbol{\eta}_{T} \partial_{i} \boldsymbol{\eta}_{T}+\partial_{i} \eta_{L} \partial_{i} \eta_{L}\right)\right. \\
& +4 c_{4}^{k} \frac{2 c^{2}-s^{2}}{r^{2}} \eta_{L} r \boldsymbol{\nabla} \boldsymbol{\eta}_{T}+m_{\pi}^{2} c \boldsymbol{\eta}^{2}-2 c_{4}^{k} F^{\prime} s\left(\eta_{L}^{2}\right)^{\prime}+4 c_{4}^{k} \frac{c\left(c^{2}-3 s^{2}\right)}{r} \eta_{L}^{2} \\
& \left.-c_{4}^{k} c\left(F^{2}+2 \frac{s^{2}}{r^{2}}\right)\left(\eta_{L}^{2}+3 \boldsymbol{\eta}_{T}^{2}\right)+2 c_{4}^{m}\left(c(2 c-1) \boldsymbol{\eta}^{2}-2 s^{2} \eta_{L}^{2}\right)\right)
\end{aligned}
$$

the e.o.m for magnetic and electric modes may be generated using the standard procedure.

From eq. (3.22) it is noticed that in the vacuum sector or equivalently far away from the soliton centre $(F \rightarrow 0, s \rightarrow 0, c \rightarrow 1)$ the $\sigma$-term does not vanish. This implies that $h_{0}^{2}(\varepsilon)$ does in general not correspond to a free Klein-Gordon equation. However, for $t=0 \quad\left(i_{0}=1\right)$ and far away from 
the soliton centre, the sole effect of the terms in (3.22) stemming from the external scalar field is to alter the asymptotical pion mass

$$
m_{\pi}^{2} \rightarrow m_{a}^{2}=\frac{m_{\pi}^{2}(1+\varepsilon)+2 c_{4}^{m} \varepsilon}{1+2 c_{4}^{k} \varepsilon}
$$

such that $h_{0}^{2}(\varepsilon)$ corresponds again to a free Klein-Gordon equation, but with the mass (3.23). This change related to the vacuum $\sigma$-term [4] is easily taken into account by just using the mass (3.23) in the expression for the Casimir energy (2.26) . Note here, that the renormalized LECs do not depend on the external field which simplifies the situation considerably.

The external scalar field $(3.16)$ is rotationally invariant and, for $t=0$, translationally invariant, too. Therefore all zero modes must be recovered in the presence of the external scalar field with $t=0$ at zero energy. This serves as a crucial test that we have solved the stability condition for $F$ and the e.o.m for the fluctuations correctly.

For $t \neq 0$, i.e. for the scalar radius $\left(i_{0}(\sqrt{t} r) \simeq 1+r^{2} t / 6\right)$ the terms proportional to the external scalar field (3.16) lead to a confining potential (oscillator potential in the case of the scalar radius) and we are not able to use the phaseshift formula (2.21) without manipulation. In coordinate space the expression $\int d^{3} r\left\langle h(\varepsilon)-h_{0}(\varepsilon)\right\rangle$ collects contributions only from a region $r \leq R$ where the soliton profile is unequal zero. We integrate the phase shifts $\delta(\varepsilon)$ and $\delta_{0}(\varepsilon)$ of $h^{2}(\varepsilon)$ and $h_{0}^{2}(\varepsilon)$, respectively, to a radius $R$ where the asymptotical mass becomes

$$
m_{a}^{2}=\frac{m_{\pi}^{2}\left(1+\varepsilon i_{0}(\sqrt{t} R)\right)+2 c_{4}^{m} \varepsilon i_{0}(\sqrt{t} R)}{1+2 c_{4}^{k} \varepsilon i_{0}(\sqrt{t} R)} .
$$

The difference $\delta(\varepsilon)-\delta_{0}(\varepsilon)$ entering the phase shift formula (2.21) should then lead to a Casimir energy independent of $R$, provided $R$ was chosen large enough.

\subsection{Axial properties}

The most general form of the nucleon axial current in the Breit frame $p^{\mu}=$ $\left(E,-\frac{1}{2} \boldsymbol{q}\right), p^{\mu \prime}=\left(E, \frac{1}{2} \boldsymbol{q}\right), E=\sqrt{M^{2}+\frac{1}{4} q^{2}}$ (no energy transfer $q^{\mu}=(0, \boldsymbol{q})$ ) is given by

$$
\begin{aligned}
\left\langle N^{\prime}\left(\frac{1}{2} \boldsymbol{q}\right)\left|A_{0}^{a}\right| N\left(-\frac{1}{2} \boldsymbol{q}\right)\right\rangle= & \left\langle N^{\prime}\left|\frac{E}{2 M^{2}} G_{T}\left(q^{2}\right)(\boldsymbol{\sigma} \boldsymbol{q}) \frac{\tau_{a}}{2}\right| N\right\rangle \\
\left\langle N^{\prime}\left(\frac{1}{2} \boldsymbol{q}\right)\left|A_{i}^{a}\right| N\left(-\frac{1}{2} \boldsymbol{q}\right)\right\rangle= & \left\langle N^{\prime}\right|\left(\frac{E}{M} G_{A}\left(q^{2}\right)\left(\sigma_{i}-\hat{q}_{i}(\hat{\boldsymbol{q}} \boldsymbol{\sigma})\right)\right. \\
& \left.+\left(G_{A}\left(q^{2}\right)-\frac{q^{2}}{4 M^{2}} G_{P}\left(q^{2}\right)\right) \hat{q}_{i}(\hat{\boldsymbol{q}} \boldsymbol{\sigma})\right) \frac{\tau_{a}}{2}|N\rangle .
\end{aligned}
$$


The time component $A_{0}^{a}$ and the corresponding pseudotensor formfactor $G_{T}\left(q^{2}\right)$ are related to that part of the axial current which has positive $G$ parity (so-called second class current). In soliton models the time component of the axial current in tree approximation

$$
\begin{aligned}
A_{0}^{a} \stackrel{\text { tree }}{=}-f_{\pi}^{2} D_{a p}\left(b_{T}+c_{4}^{f} \square\right) s c\left(\hat{\boldsymbol{r}} \times \Omega^{\boldsymbol{R}}\right)_{p} \\
\quad=\frac{f_{\pi}^{2}}{\Theta}\left(b_{T}+c_{4}^{f} \square\right) s c \varepsilon_{p i q} \hat{r}_{i} \frac{1}{2}\left\{D_{a p}, R_{q}\right\}
\end{aligned}
$$

is proportional to the angular velocity $\boldsymbol{\Omega}^{R}$ and to the right angular momentum $\boldsymbol{R}$, respectively. Because after hermitean ordering the operator

$$
\left\langle N^{\prime}\left|\varepsilon_{p i q} \frac{1}{2}\left\{D_{a p}, R_{q}\right\}\right| N\right\rangle=\frac{i}{2}\left\langle N^{\prime}\left|\boldsymbol{R}^{2} D_{a i}-D_{a i} \boldsymbol{R}^{2}\right| N\right\rangle=0
$$

vanishes between nucleon states there is no contribution to the axial pseudo tensor formfactor [18]. There may well be nonvanishing loop corrections; however, loop corrections to nonadiabatic quantities (as (3.26)) become very much involved and are not treated in this paper.

The $\mathcal{O}\left(N_{C}\right)$ spatial components of the axial current appear in soliton models as $A_{i}^{a}=D_{a p} \tilde{A}_{i}^{p}$ where the Euler angle dependent $D$-function transforms the intrinsic $\tilde{A}_{i}^{p}$ to the lab. system. For the nucleon matrix element we may use $D_{a p}=-\frac{1}{3} \tau_{a} \sigma_{p}$ and the decomposition

$$
\tilde{A}_{i}^{p}=A_{L} \hat{r}_{i} \hat{r}_{p}+A_{T}\left(\delta_{i p}-\hat{r}_{i} \hat{r}_{p}\right)
$$

into longitudinal and transversal parts to obtain

$$
\begin{aligned}
\left\langle N^{\prime}\left|\int d^{3} r e^{i \boldsymbol{q} \boldsymbol{r}} A_{i}^{a}\right| N\right\rangle= & \left\langle N^{\prime}\left|\int d^{3} r e^{i \boldsymbol{q} \boldsymbol{r}} D_{a p} \tilde{A}_{i}^{p}\right| N\right\rangle \\
= & -\frac{2}{3}\left\langle N^{\prime}\right| \int d^{3} r e^{i \boldsymbol{q} \boldsymbol{r}_{\left[A_{L}\right.} \hat{r}_{i} \hat{r}_{p}} \\
& \left.+A_{T}\left(\delta_{i p}-\hat{r}_{i} \hat{r}_{p}\right)\right] \sigma_{p} \frac{\tau_{a}}{2}|N\rangle \\
= & -\frac{2}{9}\left\langle N^{\prime}\right| \int d^{3} r\left[j_{0}(q r)\left(A_{L}+2 A_{T}\right) \sigma_{i}\right. \\
& \left.+j_{2}(q r)\left(A_{L}-A_{T}\right)\left(\sigma_{i}-3 \hat{q}_{i}(\hat{\boldsymbol{q}} \boldsymbol{\sigma})\right)\right] \frac{\tau_{a}}{2}|N\rangle
\end{aligned}
$$

The decomposition (3.28) is evident for the tree approximation (see (3.33) below) and holds still for the 1-loop part when adiabatic fluctuations depending on $\boldsymbol{r}$ and momentum $\boldsymbol{p}$ are inserted and the momentum is integrated out. Then $\tilde{A}_{i}^{p}$ is again a function of only $\boldsymbol{r}$ and the decomposition (3.28) is possible.

Comparing (3.25) and (3.29) the axial formfactor $G_{A}$ and the induced formfactor $G_{P}$ can be read off as

$$
\frac{E}{M} G_{A}\left(q^{2}\right)=-\frac{2}{9} \int d^{3} r\left[\left(j_{0}(q r)+j_{2}(q r)\right) A_{L}+\left(2 j_{0}(q r)-j_{2}(q r)\right) A_{T}\right]
$$




$$
\begin{array}{r}
G_{A}\left(q^{2}\right)-\frac{q^{2}}{4 M^{2}} G_{P}\left(q^{2}\right)=-\frac{2}{9} \int d^{3} r\left[\left(j_{0}(q r)-2 j_{2}(q r)\right) A_{L}\right. \\
\left.+2\left(j_{0}(q r)+j_{2}(q r)\right) A_{T}\right] .
\end{array}
$$

Because $G_{P}$ is not very well known experimentally we will concentrate on $G_{A}$, in particular on the axial vector coupling constant

$$
g_{A} \equiv G_{A}(0)=-\frac{2}{9} \int d^{3} r\left(A_{L}+2 A_{T}\right)=-\frac{2}{9} \int d^{3} r \tilde{A}_{i}^{i} .
$$

Relativistic corrections [57 may be accounted for through the replacement

$$
G_{A}\left(q^{2}\right) \rightarrow \frac{M}{E} G_{A}\left(\frac{M^{2} q^{2}}{E^{2}}\right)
$$

For small momentum transfers these corrections are of minor importance; $g_{A}$ is not affected at all and the axial square radius experiences only a slight increase by $3 / 4 M^{2}=0.03 \mathrm{fm}^{2}($ compare $(3.36))$.

\subsubsection{Tree approximation}

In tree approximation, the spatial components of the intrinsic axial current

$$
\tilde{A}_{i}^{p} \stackrel{\text { tree }}{=} f_{\pi}^{2}\left[\left(b_{L}+c_{4}^{f} \square\right) F^{\prime} \hat{r}_{i} \hat{r}_{p}+\left(b_{T}+c_{4}^{f} \square\right) \frac{s c}{r}\left(\delta_{i p}-\hat{r}_{i} \hat{r}_{p}\right)\right]
$$

are of the form (3.28) as already mentioned and the axial formfactor is obtained as

$$
\frac{E}{M} G_{A}\left(q^{2}\right) \stackrel{\text { tree }}{=}-\frac{2 f_{\pi}^{2}}{9} \int d^{3} r\left[\left(j_{0}+j_{2}\right)\left(b_{L}+c_{4}^{f} q^{2}\right) F^{\prime}+\left(2 j_{0}-j_{2}\right)\left(b_{T}+c_{4}^{f} q^{2}\right) \frac{s c}{r}\right] \text {. }
$$

For completeness, we give the corresponding formulae for the axial vector coupling constant and the axial square radius

$$
\begin{aligned}
& g_{A} \stackrel{\text { tree }}{=}-\frac{2 f_{\pi}^{2}}{9} \int d^{3} r\left(b_{L} F^{\prime}+2 b_{T} \frac{s c}{r}\right) \\
& <r^{2}>_{A} \stackrel{\text { tree }}{=} \frac{3}{4 M^{2}}-\frac{2 f_{\pi}^{2}}{15 g_{A}} \int d^{3} r r^{2}\left(b_{L} F^{\prime}+4 b_{T} \frac{s c}{r}\right) \\
& +c_{4}^{f} \frac{4 f_{\pi}^{2}}{3 g_{A}} \int d^{3} r\left(F^{\prime}+2 \frac{s c}{r}\right) \text {. }
\end{aligned}
$$

It should be mentioned that the main contribution to the axial radius is due to the non-minimal coupling (last term in $(3.36)$ ). In tree approximation the axial coupling constant turns out too small in chiral soliton models provided reasonable parameters are used and this deficiency can not be cured through inclusion of vector mesons. Therefore this quantity is considered to be one important candidate for which loop corrections may prove to be essential. 


\subsubsection{Loop corrections to the axial formfactor}

Here we want to consider the loop corrections to the axial formfactor (3.34). For this purpose we have to discuss the Casimir energy in the presence of an external axial field $a_{i}^{a}$,

$$
\begin{gathered}
L(\varepsilon)=L+\varepsilon \int d^{3} r a_{i}^{a} A_{i}^{a}=L+\varepsilon \int d^{3} r \tilde{a}_{i}^{p} \tilde{A}_{i}^{p}, \\
A_{i}^{a}=D_{a p} \tilde{A}_{i}^{p}, a_{i}^{a}=D_{a p} \tilde{a}_{i}^{p}
\end{gathered}
$$

which finally leads to the axial formfactor $g_{A}\left(q^{2}\right) \equiv E / M G_{A}\left(q^{2}\right)$;

$$
M(\varepsilon)=M+\varepsilon g_{A}, \quad g_{A}\left(q^{2}\right)=\left.\frac{\partial M(\varepsilon)}{\partial \varepsilon}\right|_{\varepsilon=0} .
$$

According to (3.28) and (3.30) the external axial field has to be chosen as

$$
\begin{aligned}
\tilde{a}_{i}^{p} & =\frac{2}{9}\left[\left(j_{0}+j_{2}\right) \hat{r}_{i} \hat{r}_{p}+\left(j_{0}-\frac{1}{2} j_{2}\right)\left(\delta_{i p}-\hat{r}_{i} \hat{r}_{p}\right)\right] \\
a_{i}^{a} & =\frac{2}{9}\left[\left(j_{0}+j_{2}\right) D_{a p} \hat{r}_{i} \hat{r}_{p}+\left(j_{0}-\frac{1}{2} j_{2}\right)\left(D_{a i}-D_{a p} \hat{r}_{i} \hat{r}_{p}\right)\right] .
\end{aligned}
$$

This simplifies for the axial vector coupling constant $g_{A}=g_{A}(0)$ to

$$
\tilde{a}_{i}^{p}=\frac{2}{9} \delta_{i p}, \quad a_{i}^{a}=\frac{2}{9} D_{a i}
$$

We wrote down here the axial currents and fields in the lab frame (without tilde) just to display their correct axial transformation properties although we do not need them in the following: the whole calculation is performed in the intrinsic system using the standard procedure.

If we expand the lagrangian (3.37) in the presence of the external axial field to linear order in the fluctations, we notice that again the hedgehog ansatz solves the static e.o.m . The stability condition is obtained by variation of (3.38) with the axial formfactor in tree approximation (3.34) which yields a $q$-dependent chiral angle and makes the linear term in the fluctuations vanish.

In close correspondence with the longitudinal and transversal operators appearing in the external axial field we can distinguish two pieces in the lagrangian quadratic in the fluctuations

$$
\begin{aligned}
L(\varepsilon) & \stackrel{\eta^{2}}{=} L-\frac{2 \varepsilon}{9}\left\{\int d^{3} r\left(j_{0}+j_{2}\right)\left[F^{\prime} \boldsymbol{\eta}_{T}^{2}+\cdots\right]\right. \\
& \left.+\int d^{3} r\left(j_{0}-\frac{1}{2} j_{2}\right)\left[\frac{2 s}{r} \eta_{L} r \boldsymbol{\nabla} \boldsymbol{\eta}_{T}+\frac{2 s c}{r}\left(2 \eta_{L}^{2}+\boldsymbol{\eta}_{T}^{2}\right)+\cdots\right]\right\}
\end{aligned}
$$


from which the e.o.m for the fluctuations may be generated (for the sake of simplicity we have listed only terms produced by the $\mathrm{N} \ell \sigma$ model). From eq.(3.41) it is clear that the axial current matrix element is zero in the vacuum sector $F \rightarrow 0$. Therefore $h_{0}^{2}(\varepsilon)=h_{0}^{2}$ corresponds to a free Klein-Gordon equation with pion mass $m_{\pi}$ and we do not have the difficulties discussed in the preceding section for the $\sigma$-term.

For the 1 loop calculation of $g_{A}$ there exists an alternative method concerning the evaluation of the integral (3.37) which we shall describe briefly. The divergence of the axial current is related to the symmetry breaking terms in the lagrangian (2.47)

$$
\begin{aligned}
\partial^{\mu} A_{\mu}^{a} & =\dot{A}_{0}^{a}+\partial^{i} A_{i}^{a} \\
& =m_{\pi}^{2} D_{a p}\left[f_{\pi}^{2} s \hat{r}_{p}+f_{\pi}\left(c \hat{r}_{p} \eta_{L}+\eta_{p T}\right)-\frac{s}{2} \hat{r}_{p} \eta^{2}\right]+\cdots,
\end{aligned}
$$

where we expanded the standard pion mass term up to quadratic order in the fluctuations (similarly for the other symmetry breakers not listed here). We may now use eq.(3.42) at order $\eta^{2}$ to simplify the integral in (3.37) by partial integration,

$$
\begin{aligned}
L(\varepsilon) & \stackrel{\eta^{2}}{=} L+\frac{2 \varepsilon}{9} \int d^{3} r \partial^{i} x_{p} \tilde{A}_{i}^{p} \\
& =L-\frac{2 \varepsilon}{9} \int d^{3} r x_{p} \partial^{i} \tilde{A}_{i}^{p} \\
& =L+\frac{m_{\pi}^{2} \varepsilon}{9} \int d^{3} r r s \boldsymbol{\eta}^{2}+\cdots,
\end{aligned}
$$

where we have checked that the surface terms vanish. Because we neglect the contribution from $\dot{A}_{0}^{a}$ which is $\mathcal{O}\left(N_{C}^{-1}\right)$ the loop corrections to $g_{A}$ calculated according to (3.41) and (3.43), respectively, differ just by $\mathcal{O}\left(N_{C}^{-1}\right)$.

The disturbing result is now that this difference turns out to be large in our soliton models (.45 for parameter set $A$ ) which suggests that the $\mathcal{O}\left(N_{C}^{-1}\right)$

contribution to $g_{A}$ might be large, too. The consequence of a large $\mathcal{O}\left(N_{C}^{-1}\right)$ contribution to $g_{A}$ and it's connection to current algebra will be discussed in the following subsection.

\subsubsection{Current algebra and $1 / N_{C^{-}}$expansion}

From the time component of the axial current in the infinite momentum frame and the chiral charge commutator

$$
\left[Q_{5}^{a}, Q_{5}^{b}\right]=i \varepsilon_{a b c} L_{c},
$$


Kirchbach and Riska [58] derived a model independent version of the Adler - Weisberger sum rule

$$
g_{A}^{2}=1+R .
$$

In [58], the "1", produced by the commutation relations, is of $\mathcal{O}\left(N_{C}^{0}\right)$ and $R$ is always positive.

From (3.45), it is immediately obvious that the $1 / N_{C}$ expansion cannot converge reasonably for both, $g_{A}^{2}$ and $R$, because otherwise we would expect the $\mathcal{O}\left(N_{C}^{0}\right)$ contributions to both of these quantities to be small compared to the experimental values of 1.56 and .56 respectively, which violates (3.45) at $\mathcal{O}\left(N_{C}^{0}\right)$ ! Obviously, the $1 / N_{C}$ expansion can converge rapidly, if it is to converge at all, only for one of the two quantities. From our soliton model calculations we conjecture that this is the case for $R$ and not for $g_{A}^{2}$ : For example, the model $A$ in tree +1 -loop provides the $\mathcal{O}\left(N_{C}^{2}\right)$ and $\mathcal{O}\left(N_{C}\right)$ contributions $g_{A}^{2}=R=.83-.41=.42$. Clearly, this seems to converge to the experimental value of $R$ rather than to that of $g_{A}^{2}$. Interestingly, such a result (negative $\mathcal{O}\left(N_{C}^{0}\right)$ contribution to $g_{A}$ ) was already found in [31], although we disagree with the procedure used there. If we add the current algebra "1" of $\mathcal{O}\left(N_{C}^{0}\right)$ from eq.(3.45) by hand, thereby assuming that $g_{A}$ calculated up to $\mathcal{O}\left(1 / N_{C}\right)$ exhausts relation (3.45), we obtain the quite satisfactory result $g_{A}=.91-.25+.54=1.20$ at the expense of a large $\mathcal{O}\left(N_{C}^{-1}\right)$ contribution to $g_{A}$. The suggestion that there might be a sizeable $\mathcal{O}\left(1 / N_{C}\right)$ piece contained in $g_{A}$ is supported by the alternative calculation in the preceeding subsection, which yields a large difference precisely in this order. However, an explicit calculation of this piece, which involves tree (with two angular velocities), nonadiabatic 1-loop (one angular velocity) and adiabatic 2-loop contributions seems forbiddingly complicated. Using instead the ad hoc addition of the CA "1", we may estimate the contributions to $g_{A}$ for the other parameter sets, too. The individual $1 / N_{C}$ contributions to $g_{A}$ for the various models are listed in the table 3.1. It is noticed that the $1 / N_{C}$ piece is large and positive throughout and increases the tree +1 loop value towards the experimental datum, except for the case of parameter combination $D\left(e=3.75, g_{\omega}=\right.$ 0 ), designed to yield acceptable numbers for $g_{A}$ already at tree +1 loop level at the expense of all other quantities, which turns out to considerably overestimate $g_{A}$ and should therefore be discarded.

For the axial radius we may employ the same procedure. Instead of (3.45), one has [59]

$$
g_{A}^{2}<r^{2}>_{A}=<r^{2}>_{1}^{V}+X
$$

with the isovector square radius $<r^{2}>_{1}^{V}=.58 \mathrm{fm}^{2}$ which determines the nucleon- $\Delta$ transition radius $<r^{2}>_{A}^{*}$. (Note that, in the end, we will be interested in the quantity $g_{A}<r^{2}>_{A}$ which is the slope of the formfactor). 
The isovector square radius related to the isovector charge square radius and magnetic moment

$$
<r^{2}>_{1}^{V}=<r^{2}>_{E}^{V}-\frac{3}{M^{2}}\left(\mu^{V}-\frac{1}{2}\right)
$$

appears due to the CA commutation relations and is $\mathcal{O}\left(N_{C}^{0}\right)$.

From the experimental datum $<r^{2}>_{A}=.42 \mathrm{fm}^{2}$ we again find a small value $\left[g_{A}<r^{2}>_{A}\right]^{(1+0)}=.19 \mathrm{fm}^{2}$ (superscripts here denote $N_{C}$ orders) in tree +1 loop and a large positive $1 / N_{C}$ correction $\left[g_{A}<r^{2}>_{A}\right]^{(-1)}=.34 \mathrm{fm}^{2}$. Again for the reasonable sets $(A)-(C)$ the pattern is repeated and the tree + 1 loop result enhanced toward the experimental value $g_{A}\left\langle r^{2}\right\rangle_{A}=.53 \mathrm{fm}^{2}$ although in this case there is an overestimation for the first three models and model $(D)$ is not widely off the mark. Altogether, for the axial radius, numbers are less conclusive in view of the large error of the axial radius of ${ }_{-.08}^{+.18} \mathrm{fm}^{2}$.

Table 3.1: Comparative listing of tree, 1-loop and estimated $1 / N_{C}$ piece of $g_{A}$ and $g_{A}<r^{2}>_{A}$ for the four parameter combinations $A, B, C$ and $D$.

\begin{tabular}{|c|c|c|c|c|}
\hline & $A$ & $B$ & $C$ & $D$ \\
\hline$g_{A}^{(1)}$ & .91 & .96 & 1.0 & 1.14 \\
\hline$g_{A}^{(0)}$ & -.25 & -.15 & -.11 & .14 \\
\hline$g_{A}^{(-1)}$ & .54 & .48 & .44 & .34 \\
\hline$g_{A}$ & 1.20 & 1.29 & 1.36 & 1.62 \\
\hline$\left[g_{A}<r^{2}>_{A}\right]^{(1)}\left[\mathrm{fm}^{2}\right]$ & .41 & .42 & .41 & .62 \\
\hline$\left[g_{A}<r^{2}>_{A}\right]^{(0)}\left[\mathrm{fm}^{2}\right]$ & -.13 & -.07 & .11 & -.16 \\
\hline$\left[g_{A}<r^{2}>_{A}\right]^{(-1)}\left[\mathrm{fm}^{2}\right]$ & .38 & .35 & .28 & .28 \\
\hline$\left[g_{A}<r^{2}>_{A}\right]\left[\mathrm{fm}^{2}\right]$ & .62 & .65 & .76 & .69 \\
\hline$<r^{2}>_{A}\left[\mathrm{fm}^{2}\right]$ & .55 & .54 & .59 & .46 \\
\hline
\end{tabular}

This unusual scenario (negative $\mathcal{O}\left(N_{C}^{0}\right)$ and large positive $\mathcal{O}\left(N_{C}^{-1}\right)$ contribution) for axial quantities is in striking contrast to all other models (nonrelativistic quark model, bag models, NJL model) which find a positive $\mathcal{O}\left(N_{C}^{0}\right)$ and no $\mathcal{O}\left(N_{C}^{-1}\right)$ correction. This is very serious because not only the experimental value of $g_{A}$ itself but also the individual contributions of different $N_{C}$ orders are fixed in principle and if the soliton approach is indeed correct, all others are wrong and vice versa. Interestingly, the same numbers for the quantum correction at $\mathcal{O}\left(N_{C}^{-1}\right)$ come out of a calculation which assumes 
that Skyrme type models should give a positive remainder $R$ and imposes $S U(4)$ current algebra for the spatial components of the axial current. This calculation is presented in some detail in appendix A.

As an addendum, we have calculated the scale dependence of $g_{A}$ starting from set $A$. The result, depicted in fig. 3.3 shows the scale independence to be not worse than for the mass considering the fact that the tree value (dashed line) of $g_{A}$ is much more sensitive to the change of scale than the soliton mass. Due to the too large symmetric 4 th order term which incurs numerical difficulties, we were unable to calculate the correction at scales below $600 \mathrm{MeV}$.

Figure 3.3: Scale dependence of $g_{A}$ for a model which at scale $\mu=770 \mathrm{MeV}$ corresponds to $e=4.25\left(g_{\omega}=0.0\right)$. Dashed line: Tree value, solid line: tree + 1-loop.

\subsection{Electromagnetic formfactors}

The nucleon matrix element of the electromagnetic current in the Breit frame

$$
\begin{aligned}
\left\langle N^{\prime}\left(\frac{1}{2} \boldsymbol{q}\right)\left|J_{0}(0)\right| N\left(-\frac{1}{2} \boldsymbol{q}\right)\right\rangle & =\left\langle N^{\prime}\left|G_{E}^{V}\left(q^{2}\right) \tau_{3}+G_{E}^{S}\left(q^{2}\right)\right| N\right\rangle \\
\left\langle N^{\prime}\left(\frac{1}{2} \boldsymbol{q}\right)\left|J_{i}(0)\right| N\left(-\frac{1}{2} \boldsymbol{q}\right)\right\rangle & =\left\langle N^{\prime}\left|\left[\frac{G_{M}^{V}\left(q^{2}\right)}{2 M} \tau_{3}+\frac{G_{M}^{S}\left(q^{2}\right)}{2 M}\right] i(\boldsymbol{\sigma} \times \boldsymbol{q})_{i}\right| N\right\rangle
\end{aligned}
$$


fixes the isoscalar and isovector formfactors which are linear combinations of the proton and neutron formfactors

$$
G_{E, M}^{S}=\frac{1}{2}\left(G_{E, M}^{p}+G_{E, M}^{n}\right), G_{E, M}^{V}=\frac{1}{2}\left(G_{E, M}^{p}-G_{E, M}^{n}\right)
$$

normalized to the isoscalar and isovector charges and magnetic moments

$$
G_{E}^{S}(0)=\frac{1}{2}, G_{E}^{V}(0)=\frac{1}{2}, G_{M}^{S}(0)=\mu^{S}, G_{M}^{V}(0)=\mu^{V} .
$$

The corresponding radii

$$
<r^{2}>_{E, M}^{S, V}=-\left.\frac{6}{G_{E, M}^{S, V}\left(q^{2}\right)} \frac{d G_{E, M}^{S, V}\left(q^{2}\right)}{d q^{2}}\right|_{q^{2}=0}
$$

are related to the slopes of these formfactors.

According to the charge operator $Q=\frac{1}{2}\left(\tau_{3}+1 / N_{C}\right)$ which guarantees integer charges of $\mathcal{O}\left(N_{C}^{0}\right)$ for mesons and baryons the electromagnetic current in soliton models

$$
J_{\mu}=V_{\mu}^{3}+\frac{1}{2} V_{\mu}^{0}=D_{3 p} \tilde{V}_{\mu}^{p}+\frac{1}{2} V_{\mu}^{0}
$$

decomposes into an isovector part represented by the third component of the vector current and an isoscalar part related to the baryon current. For the nucleon matrix element we may again use $D_{3 p}=-\frac{1}{3} \tau_{3} \sigma_{p}$ to obtain

$$
\left\langle N^{\prime}\left|\int d^{3} r e^{i \boldsymbol{q} \boldsymbol{r}} J_{\mu}\right| N\right\rangle=\left\langle N^{\prime}\left|\int d^{3} r e^{i \boldsymbol{q} \boldsymbol{r}}\left[-\frac{1}{3} \tau_{3} \sigma_{p} \tilde{V}_{\mu}^{p}+\frac{1}{2} V_{\mu}^{0}\right]\right| N\right\rangle .
$$

Comparison with (3.48) gives the electromagnetic formfactors

$$
\begin{aligned}
G_{E}^{V}\left(q^{2}\right) & =-\frac{1}{3} \int d^{3} r j_{0}(q r) \sigma_{p} \tilde{V}_{0}^{p} \\
G_{E}^{S}\left(q^{2}\right) & =\frac{1}{2} \int d^{3} r j_{0}(q r) V_{0}^{0} \\
\frac{G_{M}^{V}\left(q^{2}\right)}{2 M} & =\frac{1}{6} \int d^{3} r \frac{j_{1}(q r)}{q r} \varepsilon_{p i j} x_{j} \tilde{V}_{i}^{p} \\
\frac{G_{M}^{S}\left(q^{2}\right)}{2 M} & =\frac{1}{4} \int d^{3} r \frac{j_{1}(q r)}{q r}(\boldsymbol{\sigma} \times \boldsymbol{r})_{i} V_{i}^{0} .
\end{aligned}
$$

Relativistic corrections [57] may be taken into account by the replacements

$$
G_{E}^{S, V}\left(q^{2}\right) \rightarrow G_{E}^{S, V}\left(\frac{M^{2} q^{2}}{E^{2}}\right), G_{M}^{S, V}\left(q^{2}\right) \rightarrow \frac{M^{2}}{E^{2}} G_{M}^{S, V}\left(\frac{M^{2} q^{2}}{E^{2}}\right) .
$$

Again, for small momentum transfers these corrections are of minor importance; charges and magnetic moments as well as the electric radii are not affected, the magnetic square radii collect a small contribution $3 / 2 M^{2}=$ $0.06 \mathrm{fm}^{2}$. 


\subsubsection{Tree approximation}

In tree approximation we obtain for the intrinsic isovector current

$$
\begin{aligned}
& \tilde{V}_{0}^{p} \stackrel{\text { tree }}{=} \frac{f_{\pi}^{2}}{\Theta}\left(b_{T}+c_{4}^{f} \square\right) s^{2}\left\{R_{p}-\hat{r}_{p}(\hat{\boldsymbol{r}} \boldsymbol{R})\right\} \\
& \tilde{V}_{i}^{p} \stackrel{\text { tree }}{=} f_{\pi}^{2}\left(b_{T}+c_{4}^{f} \square\right) \frac{s^{2}}{r} \varepsilon_{p i j} \hat{r}_{j}
\end{aligned}
$$

and similarly for the isoscalar current

$$
\begin{aligned}
& V_{0}^{0} \stackrel{\text { tree }}{=}\left(1-\frac{1}{m_{\omega}^{2}} \square\right) B_{0}, \quad B_{0}=-\frac{1}{4 \pi^{2}} \frac{2 F^{\prime} s^{2}}{r^{2}}, \\
& V_{i}^{0} \stackrel{\text { tree }}{=}\left(1-\frac{1}{m_{\omega}^{2}} \square\right) B_{i}, \quad B_{i}=\frac{B_{0}}{\Theta}(\boldsymbol{r} \times \boldsymbol{R})_{i},
\end{aligned}
$$

where the nonminimal $C h O 6$ coupling through the $\omega$-meson, essential for the isoscalar radius, was taken into account. With the currents (3.56, 3.57) the formfactors (3.54) read in tree approximation $\left(R_{p}=-\sigma_{p} / 2\right.$ for nucleon states)

$$
\begin{aligned}
G_{E}^{V}\left(q^{2}\right) & =\frac{f_{\pi}^{2}}{3 \Theta} \int d^{3} r j_{0}(q r)\left(b_{T}+c_{4}^{f} q^{2}\right) s^{2} & G_{E}^{V}(0) & =\frac{1}{2} \\
G_{E}^{S}\left(q^{2}\right) & =\frac{1}{2}\left(1-\frac{q^{2}}{m_{\omega}^{2}}\right) \int d^{3} r j_{0}(q r) B_{0} & G_{E}^{S}(0) & =\frac{1}{2} \\
G_{M}^{V}\left(q^{2}\right) & =2 M \frac{f_{\pi}^{2}}{9} \int d^{3} r \frac{3 j_{1}(q r)}{q r}\left(b_{T}+c_{4}^{f} q^{2}\right) s^{2} & G_{M}^{V}(0) & =\mu^{V}=\frac{M}{3} \Theta \\
G_{M}^{S}\left(q^{2}\right) & =\frac{M}{6 \Theta}\left(1-\frac{q^{2}}{m_{\omega}^{2}}\right) \int d^{3} r \frac{3 j_{1}(q r)}{q r} r^{2} B_{0} & G_{M}^{S}(0) & =\mu^{S}=\frac{M}{6 \Theta}<r^{2}>_{B} .
\end{aligned}
$$

The corresponding radii are

$$
\begin{array}{ll}
<r^{2}>_{E}^{V}=<r^{2}>_{\Theta}-c_{4}^{f} \frac{4 f_{\pi}^{2}}{\Theta} \int d^{3} r s^{2}, & <r^{2}>_{\Theta}=\frac{2 f_{\pi}^{2}}{3 \Theta} \int d^{3} r r^{2} b_{T} s^{2} \\
<r^{2}>_{E}^{S}=<r^{2}>_{B}+\frac{6}{m_{\omega}^{2}}, & <r^{n}>_{B}=\int d^{3} r r^{n} B_{0} \\
<r^{2}>_{M}^{V}=\frac{3}{2 M^{2}}+\frac{3}{5}<r^{2}>_{\Theta}-c_{4}^{f} \frac{4 f_{\pi}^{2}}{\Theta} \int d^{3} r s^{2} \\
<r^{2}>_{M}^{S}=\frac{3}{2 M^{2}}+\frac{3}{5} \frac{<r^{4}>_{B}}{<r^{2}>_{B}}+\frac{6}{m_{\omega}^{2}} .
\end{array}
$$

where the relativistic correction due to (3.55) was included. The nonminimal couplings through the $\varrho$-meson in $C h O 4$ and through the $\omega$-meson in $C h O 6$ are essential and lead to a considerable enhancement of all these radii. 
In the following we will calculate loop corrections to some of these quantities. As is noticed from (3.56, 3.57) the adiabatic ones containing no angular velocity repectively no angular momentum for which the method to calculate the Casimir energy outlined above applies are the isovector magnetic formfactor of $\mathcal{O}\left(N_{C}^{1}\right)$ and the isoscalar electric formfactor of $\mathcal{O}\left(N_{C}^{0}\right)$.

\subsubsection{Loop corrections to the isovector magnetic form- factor}

For the calculation of the Casimir energy in the presence of an external isovector field $v_{i}^{a}$

$$
\begin{gathered}
L(\varepsilon)=L+\varepsilon \int d^{3} r v_{i}^{a} V_{i}^{a}=L+\varepsilon \int d^{3} r \tilde{v}_{i}^{p} \tilde{V}_{i}^{p}, \\
V_{i}^{a}=D_{a p} \tilde{V}_{i}^{p}, \quad v_{i}^{a}=D_{a p} \tilde{v}_{i}^{p}
\end{gathered}
$$

we proceed in very much the same way as for the axial field of the previous section. The external field which leads to the magnetic formfactor $g_{M}^{V}\left(q^{2}\right) \equiv$ $G_{M}^{V}\left(q^{2}\right) / 2 M$ in the form

$$
M(\varepsilon)=M+\varepsilon g_{M}^{V}, \quad g_{M}^{V}\left(q^{2}\right)=\left.\frac{\partial M(\varepsilon)}{\partial \varepsilon}\right|_{\varepsilon=0}
$$

follows from (3.54) and (3.60):

$$
\tilde{v}_{i}^{p}=\frac{3}{2} \frac{j_{1}(q r)}{q r} \varepsilon_{i p \ell} x_{\ell}, \quad v_{i}^{3}=\frac{3}{2} \frac{j_{1}(q r)}{q r} D_{3 p} \varepsilon_{i p \ell} x_{\ell} .
$$

The isovector field $v_{i}^{a}$ in the lab. frame has the correct transformation property. Again the hedgehog solves the static e.o.m. and makes the term linear in the fluctuations vanish. From the term quadratic in the fluctuations,

$$
L(\varepsilon) \stackrel{\eta^{2}}{=} L+\frac{\varepsilon}{18} \int d^{3} r \frac{3 j_{1}}{q r}\left[\left(c^{2}-s^{2}\right)\left(2 \eta_{L}^{2}+\boldsymbol{\eta}_{T}^{2}\right)+2 r c \eta_{L} \boldsymbol{\nabla} \boldsymbol{\eta}_{T}+\cdots\right]
$$

the e.o.m. for the fluctuations are set up. Here, apparently the additional terms due to the interaction do not vanish in the vacuum sector such that $h_{0}^{2}(\varepsilon)=h_{0}^{2}+\varepsilon w_{0}$ with an asymptotic potential $w_{0} \neq 0$. However, for the Casimir energy we have to evaluate $\int d^{3} r\left\langle\tilde{h}(\varepsilon)-h_{0}(\varepsilon)\right\rangle \simeq \int d^{3} r\left\langle\tilde{h}(\varepsilon)-h_{0}-\right.$ $\left.\varepsilon w_{0} / 2 h_{0}\right\rangle=\int d^{3} r\left\langle\tilde{h}(\varepsilon)-h_{0}\right\rangle$. The term which contains the asymptotic potential turns out to vanish because of $\int d^{3} r\left\langle\varepsilon w_{0} / 2 h_{0}\right\rangle=0$, which implies that the vacuum matrix element of the isovector current is zero, as it should be. 
For practical reasons, we must subtract the corresponding term which is also zero in the soliton sector from $\tilde{h}^{2}(\varepsilon)$ in order to obtain stable phase shifts.

In the presence of the vector field (3.62) which is neither rotationally nor translationally invariant (even at $q=0$ ) the e.o.m. for the fluctuations unfortunately cannot be checked by the zero modes.

\subsubsection{Loop corrections to the isoscalar formfactor}

The external isoscalar field $v_{0}^{0}=\varepsilon j_{0}(q r)$,

$$
L(\varepsilon)=L-\frac{\varepsilon}{2} \int d^{3} r v_{0}^{0} V_{0}^{0}=L-\frac{\varepsilon}{2}\left(1-\frac{q^{2}}{m_{\omega}^{2}}\right) \int d^{3} r v_{0}^{0} B_{0}
$$

immediately leads to the isoscalar electric formfactor

$$
M(\varepsilon)=M+\varepsilon G_{E}^{S}, G_{E}^{S}\left(q^{2}\right)=\left.\frac{\partial M(\varepsilon)}{\partial \varepsilon}\right|_{\varepsilon=0} .
$$

Of course, the hedgehog is again solution and the e.o.m. for the fluctuations are obtained from

$$
L(\varepsilon) \stackrel{\eta^{2}}{=} L-\frac{\varepsilon}{2}\left(1-\frac{q^{2}}{m_{\omega}^{2}}\right) \int d^{3} r j_{0}^{\prime}\left[\frac{s c}{r^{2}}\left(2 \eta_{L}^{2}+\boldsymbol{\eta}_{T}^{2}\right)+\frac{2 s}{r} \eta_{L} \boldsymbol{\nabla} \boldsymbol{\eta}_{T}\right],
$$

where we have used partial integration. It is noticed that for $q=0$ the loop contributions in (3.66) vanish identically. This is of course to be expected because the isoscalar charge $G_{E}^{S}(0)=1 / 2$ should not be subject to quantum corrections. Apart from this (trivial) test we checked the e.o.m. by the rotational zero mode, which remains at zero energy because the external field is rotationally invariant.

\subsection{Polarizabilities}

The electromagnetic polarizabilities of the nucleon have been calculated in various approaches, amongst them nonrelativistic quark- [60]-62], MIT bag[63] [64] and chiral quark models [65] as well as BChPT [6] [55] [66] [67]. The static polarizability is a measure for how easily an electric (magnetic) dipole moment may be induced by a constant external electric field $\varepsilon$ (magnetic field $\boldsymbol{b})$. In the lagrangian this leads to additional terms

$$
L(\boldsymbol{\varepsilon}, \boldsymbol{b})=L+\frac{1}{2}\left(4 \pi \alpha^{b}\right) \varepsilon^{2}+\frac{1}{2}\left(4 \pi \beta^{b}\right) \boldsymbol{b}^{2}
$$


Experimentally there is information about the dynamic polarizabilities obtained from Compton scattering [68] [69], which have to be corrected for relativistic and retardation effects caused by the finite size of the nucleon [70][73 and about the static electric polarizability of the neutron from neutron nucleus scattering [73]. There is no direct measurement of the static polarizabilities of the proton. The experimental results for the neutron and proton electric polarizabilities $\alpha^{n}=12.0 \pm 3.5 \cdot 10^{-4} \mathrm{fm}^{3}$ and $\alpha^{p}=7.0 \pm 3.5 \cdot 10^{-4} \mathrm{fm}^{3}$ are arranged into the linear combinations

$$
\begin{aligned}
\alpha & =\frac{1}{2}\left(\alpha^{n}+\alpha^{p}\right)=9.5 \pm 5.0 \cdot 10^{-4} \mathrm{fm}^{3} \\
\Delta \alpha & =\alpha^{n}-\alpha^{p}=5.0 \pm 5.0 \cdot 10^{-4} \mathrm{fm}^{3}
\end{aligned}
$$

which turn out to have different $N_{C}$ behaviour in chiral soliton models. In these models the adiabatic quantity $\alpha$ is leading $\mathcal{O}\left(N_{C}^{1}\right)$ in tree approximation (section 3.5.1) and quantum corrections of $\mathcal{O}\left(N_{C}^{0}\right)$ (section 3.5.2) may be important. The non-adiabatic quantity $\triangle \alpha$ comes with an angular velocity in tree approximation [74] and gives rise to a $\mathcal{O}\left(N_{C}^{-1}\right)$ splitting of neutron and proton electric polarizabilities (section 3.5.3). For the magnetic polarizabilities this is quite analogous, only the experimental information is much poorer there. All previous attempts to attack the problem in the framework of chiral soliton models [20] [21] [22] [24] [25] rely on the adiabatic tree approximation. Trivially there is no splitting of the neutron's and proton's electric polarizabilities $(\triangle \alpha=\triangle \beta=0)$.

More severely the numerical values obtained, in purely pseudoscalar models $\alpha \simeq 18-30 \cdot 10^{-4} \mathrm{fm}^{3}$ depending on the parameters used, come out much too large. Nonminimal couplings to the photon do not help here, they are of negligible influence (section 3.5.1). This finding is at variance with the seemingly much smaller results $\alpha=10.4-14.8 \cdot 10^{-4} \mathrm{fm}^{3}$ obtained by adding vector mesons explicitly [22] which include nonminimal couplings naturally. The reason for this discrepancy is due to $\varrho_{0}$ and $\omega_{0}$ components in the vector meson soliton profiles induced by the electric field $\varepsilon$ which lead to additional $\mathcal{O}\left(N_{C}\right)$ contributions to the electric polarizability and which are simply neglected in the approach mentioned [22]. The situation is easily understood and the missing terms can be traced by looking at the local approximation which converts the vector meson into a purly pseudoscalar model. Taking these contributions properly into account, vector meson models would end up with large electric polarizabilities comparable to those of the corresponding pseudoscalar models.

For the magnetic polarizabilities the situation is even worse. Numerical values obtained so far range from $\beta=-7-(+8.5) \cdot 10^{-4} \mathrm{fm}^{3}$ [21] 23] [24]. Up to now there does not even exist a correct and complete calculation of the leading $\mathcal{O}\left(N_{C}\right)$ contribution in the simplest Skyrme model. 


\subsubsection{Electric polarizability in tree approximation}

The isovector photon field $v_{0}^{3}=-\boldsymbol{\varepsilon} \boldsymbol{r}$ leads to a contribution in the lagrangian quadratic in the electric field $\varepsilon$ which in tree approximation is $\mathcal{O}\left(N_{C}^{1}\right)$

$$
\begin{aligned}
L_{\gamma \gamma} & =\frac{f_{\pi}^{2} e^{2}}{2} \int d^{3} r(\boldsymbol{\varepsilon} \boldsymbol{r})^{2} s^{2} b_{T} D_{3 a} D_{3 b}\left(\delta_{a b}-\hat{r}_{a} \hat{r}_{b}\right) \\
& +f_{\pi}^{2}\left(2 c_{4}^{e}-c_{4}^{f}\right) e^{2} \varepsilon^{2} \int d^{3} r s^{2} D_{3 a} D_{3 b}\left(\delta_{a b}-\hat{r}_{a} \hat{r}_{b}\right)
\end{aligned}
$$

(We use $\frac{e^{2}}{4 \pi}=\frac{1}{137}$ throughout). There is no such contribution for the isoscalar photon field and most importantly there is no $\mathcal{O}\left(N_{C}^{1}\right)$ term at all which is linear in the electric field as e.g. in vector meson models which would induce adiabatic soliton components driven by $\varepsilon$. There is, however, such a term in $\mathcal{O}\left(N_{C}^{0}\right)$, nonadiabatic, which contains an angular velocity and which consequently splits neutron and proton polarizabilities. This term is treated in subsection 3.4.3. Concluding, in leading $\mathcal{O}\left(N_{C}^{1}\right)$ eq. (3.69) represents the complete contribution to the electric polarizability

$$
\alpha=\frac{f_{\pi}^{2} e^{2}}{18 \pi} \int d^{3} r r^{2} s^{2} b_{T}+\frac{f_{\pi}^{2} e^{2}}{3 \pi}\left(2 c_{4}^{e}-c_{4}^{f}\right) \int d^{3} r s^{2}
$$

Here we used that for nucleon states $D_{3 a} D_{3 b}=\frac{1}{3} \delta_{a b}$. Alternatively we could have averaged over the orientations of the electric field $\varepsilon$ which leads to the same result. For the calculation of the quantum corrections in the next subsection we will have to do both simultaneously, taking nucleon matrix elements and averaging over the $\boldsymbol{\varepsilon}$-orientations.

The second term in (3.70) represents the non-minimal couplings. If the chiral scale is chosen such that the LECs $\ell_{6}^{r} \simeq 2 \ell_{5}^{r} \simeq-1 / 8 g^{2}$ are exhausted by the $\varrho$-meson resonance 47 then the contribution from the non-minimal couplings $c_{4}^{f} \simeq 2 c_{4}^{e}$ vanishes exactly. The Gasser Leutwyler LECs at $\mu=m_{\varrho}$ lead to an unsignificant enhancement of the electric polarizability due to the non-minimal couplings. Thus, in contrast to the common belief, neither nonminimal couplings nor the explicit inclusion of vector mesons is able to cure the problem with the much too large electric polarizabilities obtained in tree approximation from soliton models.

Such a conclusion is of course valid only if the way to calculate polarizabilities as sketched above is correct, which has recently been doubted [75] [76]. The explicit statement was that the terms quadratic in the external electric or magnetic fields (seagull terms) do not contribute at all. Since seagull terms are in fact the only contribution at leading order $N_{C}$, this claim, if true, would make quite a difference, and we shall in the following discuss it in some detail. 
A first hint that not all is well with the statement voiced in [75] [76] may be derived from the fact that the tree expression for the electric polarizability (3.70) evaluated in the chiral limit (where an analytical treatment akin to the case of the $\sigma$-term is possible) agrees with the result obtained in $H B C h P T$ [55] [56]:

$$
\alpha \stackrel{m_{\pi} \rightarrow 0}{\rightarrow} \frac{2 f_{\pi}^{2} e^{2}}{9} \int_{0}^{\infty} d r r^{4} F^{2}=\frac{e^{2}}{4 \pi} \frac{5 g_{A}^{2}}{32 \pi f_{\pi}^{2} m_{\pi}} .
$$

In order to put our concerns on a more solid footing, we insert the ansatz (2.14) for the time dependent matrix $U$ into the lagrangian coupled to the external field $\tilde{v}_{0}^{a}$ (intrinsic system). It's relevant terms then read

$$
\begin{aligned}
L(\varepsilon)=-M_{0} & +\frac{1}{2} \int d^{3} r\left(\dot{\eta}_{a} n_{a b}^{2} \dot{\eta}_{b}-\eta_{a} h_{a b}^{2} \eta_{b}\right) \\
& -\sqrt{\Theta} \int d^{3} r\left(\Omega_{e}^{R}+e \tilde{v}_{0}^{e}\right) z_{a}^{e} n_{a b}^{2} \dot{\eta}_{b} \\
& +\frac{1}{2} \Theta \int d^{3} r\left(\Omega_{e}^{R}+e \tilde{v}_{0}^{e}\right) z_{a}^{e} n_{a b}^{2} z_{b}^{f}\left(\Omega_{f}^{R}+e \tilde{v}_{0}^{f}\right) \\
& +\cdots
\end{aligned}
$$

with the moment of inertia $\Theta$ and $z_{a}^{e}$ the normalized rotational zero mode (2.36),

$$
z_{a}^{e}=\frac{f_{\pi}}{\sqrt{\Theta}} s \varepsilon_{a e i} \hat{r}_{i}
$$

The last term explicitly written down in (3.72) contains the rotational energy as well as the seagull contribution. The collective rotation (translations are unimportant in the present context) together with the fluctuations $\eta$ gives rise to a redundancy which shows up in the conjugate momenta

$$
\begin{gathered}
\pi_{a}=\frac{\delta L}{\delta \dot{\eta}_{a}}=n_{a b}^{2}\left[\dot{\eta}_{b}-\sqrt{\Theta} z_{b}^{f}\left(\Omega_{f}^{R}+e \tilde{v}_{0}^{f}\right)\right] \\
R_{e}=-\frac{\partial L}{\partial \Omega_{e}}=\sqrt{\Theta} \int d^{3} r z_{a}^{e} n_{a b}^{2} \dot{\eta}_{b}-\Theta \int d^{3} r z_{a}^{e} n_{a b}^{2} z_{b}^{f}\left(\Omega_{f}^{R}+e \tilde{v}_{0}^{f}\right)
\end{gathered}
$$

making them linearly dependent (so called primary constraint [40):

$$
R_{e}=\sqrt{\Theta} \int d^{3} r z_{a}^{e} \pi_{a} .
$$

Due to this fact, the naive Legendre transformation (used in [75] [76] ) to the hamiltonian

$$
H=M+\frac{1}{2} \int d^{3} r\left[\pi_{a} n_{a b}^{-2} \pi_{b}+\eta_{a} h_{a b}^{2} \eta_{b}\right]+e \sqrt{\Theta} \int d^{3} r \tilde{v}_{0}^{f} z_{a}^{f} \pi_{a}
$$

is not well behaved: The collective coordinates have been removed completely, and gone are the seagull term as well as the soliton's rotational energy. 
In order to keep the collective coordinates, one has to impose secondary constraints [40] conjugate to the primary ones (3.75). There is an obvious choice for these: the fluctuations should be orthogonal to the zero modes, which have already been accounted for through the collective variables

$$
\begin{aligned}
& \int d^{3} r z_{a}^{c} n_{a b}^{2} \eta_{b}=0 \\
& \int d^{3} r z_{a}^{c} n_{a b}^{2} \dot{\eta}_{b}=\int d^{3} r z_{a}^{c} \bar{\pi}_{a}=0, \quad \bar{\pi}_{a} \equiv n_{a b}^{2} \dot{\eta}_{b} .
\end{aligned}
$$

These constraints have to be added with multipliers to the lagrangian and, going through Dirac's procedure [40], one finally obtains the proper hamiltonian. However, the same objective may be achieved faster by decomposing the conjugate momenta (3.74)

$$
\pi_{a}=\pi_{a}^{c o l}+\bar{\pi}_{a}, \quad \pi_{a}^{c o l}=-\sqrt{\Theta} n_{a b}^{2} z_{b}^{f}\left(\Omega_{f}^{R}+e \tilde{v}_{0}^{f}\right)
$$

into collective and fluctuational parts and inserting them into the hamiltonian (3.76) . It is noticed that the collective motion decouples completely from the fluctuations

$$
\begin{aligned}
H & =H_{0}+H_{\pi \pi}+H_{\gamma}+H_{\gamma \gamma} \\
H_{0} & =M_{0}+\frac{\boldsymbol{R}^{2}}{2 \Theta} \\
H_{\pi \pi} & =\frac{1}{2} \int d^{3} r\left(\bar{\pi}_{a} n_{a b}^{-2} \bar{\pi}_{b}+\eta_{a} h_{a b}^{2} \eta_{b}\right) \\
H_{\gamma} & =e R_{e} \int d^{3} r z_{a}^{e} n_{a b}^{2} z_{b}^{f} \tilde{v}_{0}^{f} \\
H_{\gamma \gamma} & =\frac{e^{2}}{2} \Theta\left[\left(\int d^{3} r z_{a}^{e} n_{a b}^{2} z_{b}^{f} \tilde{v}_{0}^{f}\right)^{2}-\int d^{3} r z_{a}^{e} n_{a b}^{2} z_{b}^{f} \tilde{v}_{0}^{e} \tilde{v}_{0}^{f}\right] .
\end{aligned}
$$

The rotational energy $\boldsymbol{R}^{2} / 2 \Theta$ is recovered as well as the seagull term $H_{\gamma \gamma}$. So far we have not specialized on the particular photon field $\tilde{v}_{0}^{a}$. For a spatially constant potential $\tilde{v}_{0}^{a}=$ const., $H_{\gamma \gamma}$ does indeed vanish, as is to be expected since $\tilde{v}_{0}^{a}=$ const. corresponds to a global rotation. For the constant electric field $\tilde{v}_{0}^{a}=-\varepsilon \boldsymbol{r} D_{3 a}$ needed to calculate the electric polarizability the first integral in $H_{\gamma \gamma}$ vanishes for parity reasons and we end up with $H_{\gamma}=0$ and

$$
H_{\gamma \gamma}=-\frac{f_{\pi}^{2} e^{2}}{2} \int d^{3} r(\varepsilon \boldsymbol{r})^{2} s^{2} b_{T} D_{3 a} D_{3 b}\left(\delta_{a b}-\hat{r}_{a} \hat{r}_{b}\right)
$$

the familiar soliton model result, compare eq.(3.69). Therefore we definitely do not agree with the statement that the seagull terms should vanish and are confident that the result (3.70) for the tree contribution to the electric 
polarizabiliy in soliton models is indeed correct. Similar conclusions were independently drawn by Scoccola and Cohen 77

In the next section we will provide the method to calculate the quantum corrections to this interesting quantity in order to learn whether these 1-loop contributions are eventually able to reduce the too large tree level values.

\subsubsection{Loop corrections to the electric polarizability}

The calculation of loop corrections to the electric polarizability is very much involved, concerning both, the theoretical input and the computational effort. Although the output is only one number we decided to undergo this pain, because this quantity persistently comes out too large in tree regardless of the model considered and therefore may serve as a crucial test of the loop expansion.

In the end we want to calculate our baryon property from the Casimir energy

$$
M\left(\varepsilon^{2}\right)=M-2 \pi \varepsilon^{2} \alpha, \quad \alpha=-\left.\frac{1}{2 \pi} \frac{\partial M\left(\varepsilon^{2}\right)}{\partial \varepsilon^{2}}\right|_{\varepsilon^{2}=0}
$$

in the familiar way. For this purpose the photon field $v_{0}^{3}=-\boldsymbol{\varepsilon} \boldsymbol{r}$ has to be coupled to the lagrangian (via the covariant derivatives) which then ought to be expanded up to quadratic order in the adiabatic fluctuations. Of course if we consider a certain fixed direction of the electric field the soliton gets deformed under its influence and our whole concept, which is based on the spherical hedgehog solution would fail. Luckily we are interested only in the static electric polarizability which, defined as the coefficient of $\varepsilon^{2}$ (3.67), is independent of the direction of $\varepsilon$ and we are allowed to average over these directions. In addition we may use the relation $D_{3 a} D_{3 b}=\frac{1}{3} \delta_{a b}$ for nucleon states. With these two allowed manipulations we are able to show that the hedgehog still solves the e.o.m. in the presence of the electric field $\varepsilon$ and makes the term linear in the fluctuation vanish if the stability condition for the chiral angle is calculated by variation of the tree contribution (3.70) contained in (3.81). So far everything is quite similar to the quantities calculated in the previous sections. The complications arise with the terms quadratic in the fluctuations, or more precisely, with the e.o.m. for the fluctuations.

The lagrangian expanded to second order in the fluctuations

$$
\begin{aligned}
L(\varepsilon) & =L+L_{\gamma}+L_{\gamma \gamma} \\
L_{\gamma} & \stackrel{\eta^{2}}{=} e D_{3 a} \int d^{3} r(\varepsilon \boldsymbol{r})\left[(\boldsymbol{\eta} \times \dot{\boldsymbol{\eta}})_{a}-2(1-c) \eta_{L}(\hat{\boldsymbol{r}} \times \dot{\boldsymbol{\eta}})_{a}+\cdots\right] \\
L_{\gamma \gamma} & \stackrel{\eta^{2}}{=} \frac{1}{2} e^{2} D_{3 a} D_{3 b} \int d^{3} r(\boldsymbol{\varepsilon} \boldsymbol{r})^{2}\left[\boldsymbol{\eta}^{2} \delta_{a b}-\eta_{a} \eta_{b}-s^{2}\left(2 \eta_{L}^{2}+\boldsymbol{\eta}_{T}^{2}\right)\left(\delta_{a b}-\hat{r}_{a} \hat{r}_{b}\right)\right.
\end{aligned}
$$




$$
\left.+(1-c) \eta_{L}\left(\hat{r}_{a} \eta_{b T}+\eta_{a T} \hat{r}_{b}\right)+\cdots\right]
$$

contains a piece linear in the electric field with one time derivative and a piece quadratic in the electric field (for simplicity again only the $\mathrm{N} \ell \sigma$ contributions are listed). The second term can be simplified by using $D_{3 a} D_{3 b}=\frac{1}{3} \delta_{a b}$ for nucleon states and averaging over the electric field orientations $(\varepsilon \boldsymbol{r})^{2} \rightarrow$ $\frac{1}{3} \varepsilon^{2} r^{2}$.

Naively one might think that the first term vanishes by averaging over the electric field orientations but this is not the case for the e.o.m. as we will demonstrate in the following.

The lagrangian (3.82) introduces additional terms $\sim \varepsilon$ and $\sim \varepsilon^{2}$ into the e.o.m. which may be written formally

$$
h^{2} \eta+2 i \varepsilon w_{\gamma} \dot{\eta}-\varepsilon^{2} w_{\gamma \gamma} \eta=-n^{2}(\varepsilon) \ddot{\eta}
$$

with hermitean differential operators $w_{\gamma}$ and $w_{\gamma \gamma}$

$$
\begin{aligned}
\left(w_{\gamma}\right)_{a b}=i e D_{3 p} \hat{\boldsymbol{\varepsilon}} \boldsymbol{r}\left[\varepsilon_{p a b}-(1-c) \hat{r}_{q}\left(\hat{r}_{a} \varepsilon_{p q b}-\varepsilon_{p q a} \hat{r}_{b}\right)+\cdots\right] & (3.84) \\
\left(w_{\gamma \gamma}\right)_{a b}=e^{2} D_{3 p} D_{3 q}(\hat{\boldsymbol{\varepsilon}} \boldsymbol{r})^{2} & {\left[\delta_{p q} \delta_{a b}-\delta_{a p} \delta_{b q}-s^{2}\left(\delta_{p q}-\hat{r}_{p} \hat{r}_{q}\right)\left(\delta_{a b}+\hat{r}_{a} \hat{r}_{b}\right)\right.} \\
& \left.+(1-c)\left(\hat{r}_{a} \hat{r}_{p} \delta_{q b}+\delta_{a p} \hat{r}_{q} \hat{r}_{b}-2 \hat{r}_{a} \hat{r}_{p} \hat{r}_{q} \hat{r}_{b}\right)+\cdots\right]
\end{aligned}
$$

Differentiations appear for the higher chiral order terms omitted in (3.82). For $w_{\gamma}$ alone we obtain 9 terms of different isospin structure from the lagrangian (3.67). The single time derivative in eq.(3.83) is exactly of the form discussed in eqs. (2.33, 2.34) and consequently

$$
\frac{i}{2} \int d^{4} x\langle\ln \Omega\rangle=-\frac{T}{2} \int d^{3} r\left\langle\sqrt{\tilde{h}^{2}-\varepsilon^{2}\left(\tilde{w}_{\gamma \gamma}-\tilde{w}_{\gamma}^{2}\right)}\right\rangle
$$

there is no term linear in the electric field $\varepsilon$. Thus considering the trace log (3.85) we may equally well solve the e.o.m.

$$
h^{2} \eta-\varepsilon^{2}\left(w_{\gamma \gamma}-w_{\gamma} n^{-2} w_{\gamma}\right) \eta=-n^{2} \ddot{\eta}
$$

instead of (3.83). This is of the standard form with an additional potential, simple only in the case of the $\mathrm{N} \ell \sigma$ model $(n \equiv 1)$

$$
\begin{aligned}
\left(w_{\gamma} n^{-2} w_{\gamma}\right)_{a b}=e^{2} D_{3 p} D_{3 q}(\hat{\varepsilon} \boldsymbol{r})^{2} & {\left[\delta_{p q} \delta_{a b}-\delta_{a p} \delta_{b q}-s^{2} \hat{r}_{i} \hat{r}_{j} \varepsilon_{p i a} \varepsilon_{q j b}\right.} \\
& -s^{2} \delta_{p q} \hat{r}_{a} \hat{r}_{b}+(1-c)\left(\hat{r}_{a} \hat{r}_{p} \delta_{q b}+\delta_{a p} \hat{r}_{q} \hat{r}_{b}\right. \\
& \left.\left.-(1-c)^{2} \hat{r}_{a} \hat{r}_{p} \hat{r}_{q} \hat{r}_{b}\right)+\cdots\right]
\end{aligned}
$$

In general we had to work out, according to (3.85) and the longitudinal and transversal metric, $2 \times 9 \times 9$ terms. From (3.85) and (3.87) it is recognised 
that in contrast to the individual potentials $w_{\gamma \gamma}$ and $w_{\gamma} n^{-2} w_{\gamma}$ their difference is well behaved for $r \rightarrow \infty(F \rightarrow 0)$.

Finally we may simplify the potentials using again $D_{3 p} D_{3 q}=\frac{1}{3} \delta_{p q}$ and averaging over $\varepsilon$-field orientations to obtain

$$
\begin{aligned}
\left(w_{\gamma \gamma}\right)_{a b} & =\frac{e^{2}}{9} r^{2}\left[2 \delta_{a b}-2 s^{2}\left(\delta_{a b}+\hat{r}_{a} \hat{r}_{b}\right)+\cdots\right] \\
\left(w_{\gamma} n^{-2} w_{\gamma}\right)_{a b} & =\frac{e^{2}}{9} r^{2}\left[2 \delta_{a b}-s^{2}\left(\delta_{a b}+\hat{r}_{a} \hat{r}_{b}\right)+\cdots\right] .
\end{aligned}
$$

With $w_{\gamma \gamma}$ alone we would end up with an infinite loop correction to the polarizability of the vacuum which of course should be zero. This is how we noticed that we need the first term in (3.82) linear in the electric field in order to get a reasonable finite result for the quantum correction to the electric polarizability in the vacuum as well as in the soliton sector.

\subsubsection{Neutron-proton split of the electric polarizability}

In this subsection we present a derivation of the neutron-proton split $\Delta \alpha$ of the electric polarizability in chiral soliton models. A similar approach for a chiral soliton model with quarks was published by Broniowski et al. [74].

The quantity under investigation must contain an angular velocity in order to split the values for neutron and proton and is therefore of lower order $N_{C}$ compared to the tree and 1-loop contributions to the electric polarizability discussed in the previous sections.

We consider the term linear in the electric field (non-minimal couplings do not contribute here)

$$
L_{\gamma}=e \int d^{3} r(\varepsilon \boldsymbol{r})\left(D_{3 a} \tilde{V}_{0}^{a}+\frac{1}{2} B_{0}\right)
$$

which provides the driving terms for static fluctuations $\boldsymbol{\eta}^{S}$ and $\boldsymbol{\eta}^{V}$ induced by the isoscalar and isovector electric field (the terms linear in the fluctuations do not vanish)' To guarantee these fluctuations to be orthogonal on the zero modes $z_{a}^{c}$ we have to implement the constraints

$$
\int d^{3} r z_{a}^{c} n_{a b}^{2} \eta_{b}^{S, V}=0 \text { for all } c
$$

simply by adding them with Lagrange multipliers $\lambda_{c}^{S}$ and $\lambda_{c}^{V}$ to the lagrangian. The relevant zero modes are the three infinitesimal translations

$$
z_{a}^{c}=\frac{f_{\pi}}{\sqrt{M_{0}}}\left[F^{\prime} \hat{r}_{a} \hat{r}_{c}+\frac{s}{r}\left(\delta_{a c}-\hat{r}_{a} \hat{r}_{c}\right)\right]
$$

\footnotetext{
${ }^{1}$ This was first noticed by B. Schwesinger
} 
normalized appropriately (2.36) with $M_{0}$ being the classical soliton mass (3.3). Mathematically fluctuations unquestionably have to be defined in the space orthogonal to the one spanned by the collective coordinates (Dirac constraints), physically the constraints (3.90) make sure that simple translations of the system may not contribute to the electric polarizability.

With these preparations we are ready to solve the e.o.m.

$$
\begin{gathered}
h_{a b}^{2} \eta_{b}^{S}=\left.\frac{e}{2}(\boldsymbol{\varepsilon} \boldsymbol{r}) \frac{\delta B_{0}}{\delta \eta_{a}}\right|_{\boldsymbol{\eta}_{=0}}+\lambda_{c}^{S} n_{a b}^{2} z_{b}^{c} \\
h_{a b}^{2} \eta_{b}^{V}=\left.e(\boldsymbol{\varepsilon} \boldsymbol{r}) D_{3 p} \frac{\delta \tilde{V}_{0}^{p}}{\delta \eta_{a}}\right|_{\boldsymbol{\eta}=0}+\lambda_{c}^{V} n_{a b}^{2} z_{b}^{c}
\end{gathered}
$$

for the components induced by the electric field, which reinserted into the lagrangian

$$
\begin{aligned}
L_{\gamma \gamma} & =\int d^{3} r\left(\eta_{a}^{S}+\eta_{a}^{V}\right)\left[-\frac{1}{2} h_{a b}^{2}\left(\eta_{b}^{S}+\eta_{b}^{V}\right)+\left.e(\boldsymbol{\varepsilon} \boldsymbol{r})\left(D_{3 p} \frac{\delta \tilde{V}_{0}^{p}}{\delta \eta_{a}}+\frac{1}{2} \frac{\delta B_{0}}{\delta \eta_{a}}\right)\right|_{\boldsymbol{\eta}_{=0}}\right] \\
& =\left.\frac{e}{2} \int d^{3} r(\varepsilon \boldsymbol{r})\left(\eta_{a}^{S}+\eta_{a}^{V}\right)\left(D_{3 p} \frac{\delta \tilde{V}_{0}^{p}}{\delta \eta_{a}}+\frac{1}{2} \frac{\delta B_{0}}{\delta \eta_{a}}\right)\right|_{\boldsymbol{\eta}=0} \\
& \equiv L_{\gamma \gamma}^{S S}+L_{\gamma \gamma}^{S V}+L_{\gamma \gamma}^{V V}
\end{aligned}
$$

lead to terms proportional to $\varepsilon^{2}$ which contribute to the electric polarizability. By looking at the Legendre transformation we made sure that this naive insertion into the lagrangian is here as an exception of the rule correct.

All three terms in (3.94) are $\mathcal{O}\left(N_{c}^{-1}\right), L_{\gamma \gamma}^{S S}$ and $L_{\gamma \gamma}^{V V}$ contribute to $\alpha$ and only $L_{\gamma \gamma}^{S V}$ contains an angular velocity which splits neutron and proton electric polarizabilities. In contrast to $\alpha$ where we have many other contributions in $\mathcal{O}\left(N_{C}^{-1}\right)$ (e.g. 2-loops) the leading contribution to $\triangle \alpha$ is contained in (3.94)

$$
\begin{aligned}
L_{\gamma \gamma}^{S V} & =\left.e D_{3 p} \int d^{3} r(\varepsilon \boldsymbol{r}) \frac{\delta \tilde{V}_{0}^{p}}{\delta \eta_{a}}\right|_{\boldsymbol{\eta}_{=0}} \eta_{a}^{S} \\
& =\left.\frac{e}{2} \int d^{3} r(\boldsymbol{\varepsilon} \boldsymbol{r}) \frac{\delta B_{0}}{\delta \eta_{a}}\right|_{\boldsymbol{\eta}=0} \eta_{a}^{V} .
\end{aligned}
$$

Although we have calculated both isoscalar and isovector induced components and checked our calculation using the equivalence of the two expressions in (3.95) which follows from the e.o.m., we shall present here only the evaluation via the first one using the isoscalar component in some detail. 
Figure 3.4: Radial functions $u(r)$ and $v(r)$ of the induced isoscalar electric dipole mode plotted over a range of $3 \mathrm{fm}$. The node of $v(r)$ is due to orthogonality on the translation.

With $B_{0}$ expanded linearly in the fluctations, the e.o.m. for the induced isoscalar component follows from (3.92):

$$
h_{a b}^{2} \eta_{b}^{S}=\frac{e}{4 \pi^{2} f_{\pi}}\left[\frac{F^{\prime} s}{r}\left(\varepsilon_{a}-\hat{r}_{a}(\hat{\boldsymbol{r}} \varepsilon)\right)+\frac{s^{2}}{r^{2}} \hat{r}_{a}(\hat{\boldsymbol{r}} \varepsilon)\right]+\lambda_{c}^{S} n_{a b}^{2} z_{b}^{c} .
$$

The Lagrange multipliers $\lambda_{c}^{S}=\frac{1}{2} e \varepsilon_{c} / \sqrt{M_{0}}$, determined by multiplying (3.96) with $z_{a}^{d}$ from the left are inserted into the e.o.m.

$$
\begin{aligned}
h_{a b}^{2} \eta_{b}^{S}=\frac{e}{2 f_{\pi}} & {\left[\left(\frac{F^{\prime} s}{2 \pi^{2} r}+\frac{f_{\pi}^{2}}{M_{0}} \frac{s}{r} b_{T}\right)\left(\varepsilon_{a}-\hat{r}_{a}(\hat{\boldsymbol{r}} \varepsilon)\right)\right.} \\
& \left.+\left(\frac{s^{2}}{2 \pi^{2} r^{2}}+\frac{f_{\pi}^{2}}{M_{0}} F^{\prime} b_{L}\right) \hat{r}_{a}(\hat{\boldsymbol{r}} \varepsilon)\right] .
\end{aligned}
$$

From this equation it is noticed that the electric field induces in this case a pure $E 1$ mode

$$
\boldsymbol{\eta}^{S}=-\frac{e}{4 \pi^{2} f_{\pi}^{2}}[u(r) \hat{\boldsymbol{r}}(\hat{\boldsymbol{r}} \boldsymbol{\varepsilon})+v(r)(\boldsymbol{\varepsilon}-\hat{\boldsymbol{r}}(\hat{\boldsymbol{r}} \boldsymbol{\varepsilon}))]
$$

and it is evident that it is the translational zero mode which enters the constraints (3.90) . The radial functions $u(r)$ and $v(r)$, subject to boundary 
conditions $u^{\prime}(0)=v^{\prime}(0)=0, u(\infty)=v(\infty)=0$, are depicted in fig. 3.4; $v(r)$ has a node which is caused by orthogonality on the translational zero mode.

The $E 1$ mode $\eta^{S}$ yields after averaging over the electric field directions (first expression in (3.95) )

$$
\begin{gathered}
L_{\gamma \gamma}^{S V}=\frac{1}{2}(4 \pi \Theta \Delta \alpha) D_{3 a} \Omega_{a}^{R} \varepsilon^{2}=-\frac{1}{2}(4 \pi \Delta \alpha) L_{3} \varepsilon^{2} \\
\triangle \alpha=\frac{2 e^{2}}{9 \pi^{2} f_{\pi} \Theta} \int_{0}^{\infty} d r r^{3}\left[s c b_{T} u+\left(c_{4}^{a}-4 c_{4}^{s}+c_{6}{F^{\prime}}^{2}\right) \frac{s^{3}}{r^{2}}(c u-v)\right. \\
\left.-c_{4}^{k} s^{3} u+\left(c_{4}^{a}-2 c_{4}^{s}+c_{6} \frac{s^{2}}{r^{2}}\right) F^{\prime} s^{2} u^{\prime}\right] .
\end{gathered}
$$

Here we used that the isospin $L_{3}=-\Theta D_{3 a} \Omega_{a}^{R}$ is related to the angular momentum $\Omega_{a}^{R}$.

After presenting the mechanism which splits neutron and proton electric polarizabilities with a positive $\triangle \alpha$ of $\mathcal{O}\left(N_{C}^{-1}\right)$ we will give an analytical result in the chiral limit $m_{\pi} \rightarrow 0$. It turns out that $\Delta \alpha$ diverges in this limit such that the integral in (3.99) becomes dominated by the asymptotic region for which we are able to solve the differential equations (3.97) analytically

$$
u \stackrel{r \rightarrow \infty}{=} \frac{3 \pi f_{\pi} g_{A}}{8 M_{0} r}\left(2+m_{\pi} r\right) e^{-m_{\pi} r}, \quad v \stackrel{r \rightarrow \infty}{=} \frac{3 \pi f_{\pi} g_{A}}{8 M_{0} r} e^{-m_{\pi} r}
$$

With these solutions we obtain

$$
\triangle \alpha \stackrel{m_{\pi} \rightarrow 0}{\rightarrow} \frac{2 e^{2}}{9 \pi^{2} f_{\pi} \Theta} \int_{0}^{\infty} d r r^{3} F u=\frac{e^{2}}{4 \pi} \frac{g_{A}^{2}}{6 \pi f_{\pi}^{2} m_{\pi}} \frac{\Delta}{M_{0}},
$$

where $\Delta$ represents the nucleon- $\Delta$ split. Physical values for $g_{A}, f_{\pi}, M_{0}, \Delta$ and $m_{\pi}$ result in $\triangle \alpha=12.5 \cdot 10^{-4} \mathrm{fm}^{3}$, which is too large. However, the ratio

$$
\frac{\Delta \alpha}{\alpha} \stackrel{m_{\pi} \rightarrow 0}{\rightarrow} \frac{16}{15} \frac{\Delta}{M_{0}} \simeq \frac{1}{3}
$$

turns out to be not unreasonable. Numerical values for the exact expression (3.99) are given in the results chapter.

\subsubsection{Magnetic polarizability}

Finally we will present a complete calculation of the static magnetic polarizability $\beta$ to leading order $\mathcal{O}\left(N_{C}^{1}\right)$. All previous attempts [21 23] 24] miss an important part which may be noticed by the violation of the $H B C h P T$ result $\beta \stackrel{m_{\pi} \rightarrow 0}{=} \alpha / 10$ [55]. In the course of our derivation we will recover this 
relation although unfortunately in the end we will loose it again for reasons which will become obvious. Consequently the magnetic polarizability is the only quantity where the $H B C h P T$ result in the chiral limit is not contained in our tree approximation.

There is agreement on the contribution due to the seagull term (see discussion in section 3.5.1) which is the term quadratic in the magnetic field $\boldsymbol{b}$ when the isovector photon field $v_{i}^{3}=\frac{1}{2}(\boldsymbol{r} \times \boldsymbol{b})_{i}$ is inserted into the lagrangian

$$
\begin{aligned}
L_{\gamma \gamma}= & -\frac{f_{\pi}^{2} e^{2}}{120} D_{3 a} D_{3 b} \int d^{3} r r^{2} s^{2}\left[\left(b_{T}-c_{4}^{a} \frac{s^{2}}{r^{2}}\right)\left(6 \boldsymbol{b}^{2} \delta_{a b}+2 b_{a} b_{b}\right)\right. \\
& \left.+\left(c_{4}^{a}-2 c_{4}^{s}\right) \frac{s^{2}}{r^{2}}\left(\boldsymbol{b}^{2} \delta_{a b}+7 b_{a} b_{b}\right)\right]-\frac{2 f_{\pi}^{2} e^{2}}{3}\left(2 c_{4}^{e}-c_{4}^{f}\right) \int d^{3} r s^{2} \boldsymbol{b}^{2} .
\end{aligned}
$$

Using $D_{3 a} D_{3 b}=\frac{1}{3} \delta_{a b}$ for nucleon states we obtain the direct $\mathcal{O}\left(N_{C}^{1}\right)$ contribution

$$
\begin{aligned}
\beta_{d i r}= & -\frac{f_{\pi}^{2} e^{2}}{36 \pi} \int d^{3} r r^{2} s^{2}\left[b_{T}-\frac{1}{2}\left(c_{4}^{a}-2 c_{4}^{s}\right) \frac{s^{2}}{r^{2}}\right] \\
& -\frac{f_{\pi}^{2} e^{2}}{3 \pi}\left(2 c_{4}^{e}-c_{4}^{f}\right) \int d^{3} r s^{2}
\end{aligned}
$$

to the magnetic polarizability. The last term again represents the nonminimal couplings which vanish for $c_{4}^{f}=2 c_{4}^{e}$. In the chiral limit we obtain $\beta_{\text {dir }} \stackrel{m_{\pi} \rightarrow 0}{=}-\alpha / 2$ (compare (3.71)) which is not the desired HBChPT result indicating a missing contribution.

In analogy to (3.89) we consider the term linear in the magnetic field (non-minimal couplings do not contribute)

$$
L_{\gamma}=\frac{e}{2} \int d^{3} r(\boldsymbol{r} \times \boldsymbol{b})_{i}\left(D_{3 a} \tilde{V}_{i}^{a}+\frac{1}{2} B_{i}\right) .
$$

Because the magnetic field couples via the spatial components of the vector current, the isovector part is leading $\mathcal{O}\left(N_{C}^{1}\right)$ in contrast to the electric field case in (3.89) (but similar to the coupling of the electric field in vector meson lagrangians) and will therefore give a contribution to the magnetic polarizability in leading order. As in the electric case of the previous subsection the isoscalar part of $\mathcal{O}\left(N_{C}^{-1}\right)$ splits the polarizabilities of neutron and proton. In the following we concentrate on the leading $\mathcal{O}\left(N_{C}^{1}\right)$ term.

Previous attempts consider this term with the spherical hedgehog inserted

$$
L_{\gamma}=-\frac{3 e}{2 M} \mu^{V} D_{3 p} b_{p}
$$


in second order perturbation theory which leads to the standard paramagnetic $\Delta$ contribution

$$
\beta_{\text {para }}=\frac{e^{2}}{4 \pi M^{2}} \frac{\left(\mu^{V}\right)^{2}}{M_{\Delta}-M_{N}} .
$$

This procedure overestimates the effect of (3.105) considerably (see table 3.2) because it neglects the soliton's response to the external magnetic field. Although $\beta_{\text {para }}$ has the correct $\mathcal{O}\left(N_{C}^{1}\right)$ it remains finite in the chiral limit and cannot add to the relation $\beta \stackrel{m_{\pi \rightarrow 0}}{=}-\alpha / 2$. Therefore it is not the desired term we are looking for.

The magnetic field in (3.105) gives rise to deformations of the soliton. Because we are able to make the magnetic field arbitrarily small, it suffices to calculate the linear response via the vector current in (3.105) which provides the driving term for the static fluctuation

$$
\begin{aligned}
h_{a b}^{2} \eta_{b}^{V}= & \left.\frac{e}{2}(\boldsymbol{r} \times \boldsymbol{b})_{i} D_{3 p} \frac{\delta \tilde{V}_{i}^{p}}{\delta \eta_{a}}\right|_{\boldsymbol{\eta}=0}+\lambda_{c}^{V} n_{a b}^{2} z_{b}^{c} \\
= & -\frac{e f_{\pi}}{2} D_{3 p}\left[2 s c \hat{r}_{a}\left(b_{p}-\hat{r}_{p}(\hat{\boldsymbol{r}} \boldsymbol{b})\right)-2 s \hat{r}_{p}\left(b_{a}-\hat{r}_{a}(\hat{\boldsymbol{r}} \boldsymbol{b})\right)+\cdots\right] \\
& +\lambda_{c}^{V} n_{a b}^{2} z_{b}^{c} .
\end{aligned}
$$

The $\mathrm{N} \ell \sigma$ model terms are listed explicitly and the zero mode constraints (compare previous subsection) are added for later convenience. The solution reinserted into the lagrangian leads

$$
\begin{aligned}
L_{\gamma \gamma}^{V V} & =\int d^{3} r \eta_{a}^{V}\left[-\frac{1}{2} h_{a b}^{2} \eta_{b}^{V}+\left.\frac{e}{2}(\boldsymbol{r} \times \boldsymbol{b})_{i} D_{3 p} \frac{\delta \tilde{V}_{i}^{p}}{\delta \eta_{a}}\right|_{\boldsymbol{\eta}_{=0}}\right] \\
& =-\frac{e f_{\pi}}{4} D_{3 p} \int d^{3} r\left[2 s c \eta_{L}\left(b_{p}-\hat{r}_{p}(\hat{\boldsymbol{r}} \boldsymbol{b})\right)-2 s \hat{r}_{p}\left(\boldsymbol{b} \boldsymbol{\eta}_{\boldsymbol{T}}\right)+\cdots\right] \\
& =\frac{1}{2}\left(4 \pi \beta_{i n d}\right) \boldsymbol{b}^{2}
\end{aligned}
$$

to a term proportional to $\boldsymbol{b}^{2}$. Lets forget about the constraint for a moment and solve (3.108) analytically in the asymptotical region. The result

$$
\eta_{a}^{V} \stackrel{r \rightarrow \infty}{=} \frac{f_{\pi} e}{2}\left(\frac{3 g_{A}}{8 \pi f_{\pi}^{2}}\right) e^{-m_{\pi} r} D_{3 p}\left(\hat{r}_{p} b_{a}-\hat{r}_{a} b_{p}\right)
$$

inserted into (3.109) leads after some simple integrations to $\beta \stackrel{m_{\pi} \rightarrow 0}{=}-3 \alpha / 5$ which is exactly the $H B C h P T$ result $\beta=\beta_{\text {dir }}+\beta_{\text {ind }} \stackrel{m_{\pi} \rightarrow 0}{=} \alpha / 10$ as promised. However the full equation (3.108) cannot be solved without the constraint 
because the rotational zero mode is solution to the homogeneous equation. More explicitly this is seen by multiplying (3.108) with $z_{a}^{d}$ from the left

$$
\lambda_{c}^{V}=-\frac{e}{2} \sqrt{\Theta} D_{3 p} \epsilon_{c p q} b_{q}
$$

which determines the lagrangian mulipiers $\lambda_{c}^{V} \neq 0$. It is worth noting that (3.111) does not only hold for the $\mathrm{N} \ell \sigma$ model but also for the full lagrangian. Apart from the mathematical necessity of the constraints in eq. (3.108) they are also needed for physical reasons: we do not want simple rotations of the soliton contribute to the magnetic polarizability. When the lagrangian multipliers (3.111) are inserted into the e.o.m. (3.108)

$$
\begin{aligned}
h_{a b}^{2} \eta_{b}^{V}= & -\frac{e f_{\pi}}{2} D_{3 p}\left[2 s c \hat{r}_{a}\left(b_{p}-\hat{r}_{p}(\hat{\boldsymbol{r}} \boldsymbol{b})\right)-s\left(\delta_{a p}(\hat{\boldsymbol{r}} \boldsymbol{b})+b_{a} \hat{r}_{p}-2 \hat{r}_{a} \hat{r}_{p}(\hat{\boldsymbol{r}} \boldsymbol{b})\right)\right. \\
& +\cdots]
\end{aligned}
$$

it is noticed that the magnetic field induces a monopole and quadrupole deformation

$$
\begin{aligned}
\eta_{L}^{V} & =-\frac{e}{6 f_{\pi}} D_{3 p}\left[2 f(r) b_{p}+u(r)\left(b_{p}-3 \hat{r}_{p}(\hat{\boldsymbol{r}} \boldsymbol{b})\right)\right] \\
\eta_{a T}^{V} & =\frac{e}{4 f_{\pi}} D_{3 p} v(r)\left[\delta_{a p}(\hat{\boldsymbol{r}} \boldsymbol{b})+\hat{r}_{p} b_{a}-2 \hat{r}_{a} \hat{r}_{p}(\hat{\boldsymbol{r} b})\right]
\end{aligned}
$$

while the magnetic dipole is projected completely from the e.o.m. (3.108) by the constraint. The radial functions subject to the boundary conditions $f(0)=u(0)=v(0)=0$ and

$$
f(r) \stackrel{r \rightarrow \infty}{=} u(r) \stackrel{r \rightarrow \infty}{=} v(r) \stackrel{r \rightarrow \infty}{=} \frac{3 g_{A}}{8 \pi} e^{-m_{\pi} r}
$$

(compare (3.110)) are depicted in fig. 3.5. Because of the constraint we now obtain $\beta_{\text {ind }} \stackrel{m_{\underline{\pi}} \rightarrow 0}{=} 9 \alpha / 20$ and $\beta^{\stackrel{m_{\pi \rightarrow 0}}{=}}-\alpha / 20$ in the chiral limit in variance with the $H B C h P T$ result [55] [66]. We could not decide whether this discrepancy is located in $H B C h P T$ which does not know zero modes, or is caused by the soliton model's rigid rotator approach which rotates the profile rigidly for all distances even for $r \rightarrow \infty$.

Table 3.2 lists the complete leading $\mathcal{O}\left(N_{C}^{1}\right)$ contributions for the parameter sets $A, B$, and $C$. For comparison we also give the perturbation theory result $\beta_{\text {para }}$ (3.107) which overestimates $\beta_{\text {ind }}$ considerably and may at best be considered a rough estimate. From this table it is noticed that the total magnetic polarizability comes out small in magnitude for all three parameter sets due to a strong cancellation of the direct and induced contributions. Due to this cancellation in leading $\mathcal{O}\left(N_{C}^{1}\right)$, 1-loop corrections of $\mathcal{O}\left(N_{C}^{0}\right)$ may play a decisive role for this quantity. 
Figure 3.5: Radial functions $f(r)$ of the monopole deformation and $u(r)$ and $v(r)$ of the quadrupole deformation induced by a constant magnetic field (full lines). For comparison the asymptotical function relevant in the chiral limit is also plotted (dashed line).

However loop corrections for the magnetic polarizabilities are not presented in this paper for the following reasons: even if we were able to calculate the 1-loop contributions to $\beta_{\text {dir }}$ (3.104), where we expect a drastic reduction of the absolute tree value similar to the electric polarizability in subsection 3.5.2, we are not yet in a position to calcuate loop corrections to a deformed soliton necessary for $\beta_{\text {ind }}$. Because both loop corrections are of the same $\mathcal{O}\left(N_{C}^{0}\right)$, it does not make sense to consider only one of them alone, still it is likely that the loop corrections will shift the magnetic polarizability in table 3.2 to small positive values.

Because experimental information on static polarizabilities is scarce, it would be appropriate to calculate directly the Compton scattering amplitudes within chiral soliton models in order to avoid the model dependent and uncertain extraction of the static polarizabilities from these data. In principle this should be possible. 
Table 3.2: Direct and induced contributions to the magnetic polarizabilities are compared for the three parameter combinations $A, B, C$. The perturbation theory result $\beta_{\text {para }}$ overestimates $\beta_{\text {ind }}$ by a large margin. All polarizabilities are given in units $10^{-4} \mathrm{fm}^{3}$.

\begin{tabular}{|c|c|c|c|}
\hline & $A$ & $B$ & $C$ \\
\hline$\beta_{\text {dir }}$ & -8.6 & -9.4 & -10.7 \\
\hline$\beta_{\text {ind }}(\mathrm{E} 0)$ & 2.7 & 2.5 & 2.0 \\
$\beta_{\text {ind }}(\mathrm{E} 2)$ & 5.4 & 5.9 & 6.8 \\
$\beta_{\text {ind }}$ & 8.1 & 8.4 & 8.8 \\
\hline$\beta_{\text {para }}$ & 11.0 & 15.1 & 23.4 \\
\hline$\beta$ & -0.5 & -1.0 & -1.9 \\
\hline
\end{tabular}

\subsection{Electromagnetic properties of the $\Delta$ iso- bar}

To complete our discussion of electromagnetic properties we briefly adress some of these quantities connected with the $\Delta$ isobar. In general the static properties of the $\Delta$ are related to those of the nucleon by simple geometrical coefficients which follow from the collective operators involved. For adiabatic quantities this operator structure remains unaffected by 1-loop corrections and consequently the model independent relations survive for these quantities. The experimental verifications of these relations may be considered as a crucial test of the validity of the $1 / N_{C}$ expansion. As an example we discuss the magnetic moments in the following paragraph. There we also mention the quadrupole moment (which vanishes for the nucleon) as well as the corresponding transition moments.

Another interesting quantity subject to recent measurements is discussed in the second subsection, namely the electromagnetic ratio of the $\Delta$ photodecay amplitudes. All soliton model attempts to calculate this quantity so far make use of Siegert's theorem at the finite photon momentum where it is not stictly valid. We show that the correct treatment reduces the electromagnetic ratio considerably. 


\subsubsection{Magnetic and quadrupole moments}

The operators for the calculation of magnetic and quadrupole moments are given by

$$
\begin{aligned}
\mu & =-3 \mu^{V} D_{33}-2 \mu^{S} R_{3}+\mathcal{O}\left(N_{C}^{-1}\right) \\
Q & =-\frac{1}{5}<r^{2}>_{\Theta}\left(D_{33} R_{3}-\frac{1}{3} D_{3 p} R_{p}\right),
\end{aligned}
$$

with the isoscalar and isovector magnetic moments $\mu^{S}$ and $\mu^{V}$ and the squared radius $<r^{2}>_{\Theta}$ of the moment of inertia's density defined in section 3.4. The adiabatic quantity $\mu^{V}$ may contain the 1-loop corrections described in the section just mentioned. Evaluating the matrix elements of $\mu$ (3.115) with respect to $\Delta$ states or nucleon and $\Delta$ states

$$
\begin{aligned}
\mu^{\Delta^{++}} & =<\Delta^{++}, S_{3}=\frac{3}{2}|\mu| \Delta^{++}, S_{3}=\frac{3}{2}>=\frac{9}{5} \mu^{V}+3 \mu^{S}+\mathcal{O}\left(N_{C}^{-1}\right) \\
\mu^{N \Delta} & =<\Delta^{+}, S_{3}=\frac{1}{2}|\mu| p, S_{3}=\frac{1}{2}>=\sqrt{2} \mu^{V}+\mathcal{O}\left(N_{C}^{-1}\right)
\end{aligned}
$$

relates the magnetic (transition) moments by simple geometrical coefficients to the isoscalar and isovector magnetic moments. Upon using the experimental values $\mu^{V}=2.35$ and $\mu^{S}=.44$ we obtain $\mu^{\Delta^{++}}=5.55$ and $\mu^{N \Delta}=3.32$. If accurate experimental information about these magnetic moments were available we had a check on the $1 / N_{C}$ expansion i.e. we could estimate the next-to-next to leading order contribution generated by the vector current. In our model such $\mathcal{O}\left(N_{C}^{-1}\right)$ contributions are produced in tree approximation by small soliton deformations proportional to the angular velocity squared (next subsection), by nonadiabatic 1-loop processes which come with one angular velocity, and by 2-loop diagrams (compare a similar discussion for the axial current in appendix A). Unfortunately there are no precise experimental data: for $\mu^{\Delta^{++}}$the particle data group gives a range of $3.7-7.5$ nuclear magnetons [78, for the transition magnetic moment there exist photoproduction data, but these may not directly be compared to the matrix element (3.116) (see table 3.4).

Many other $\Delta$ observables like rms radii and polarizabilities are related to those of the nucleon by simple geometrical coefficients just as in the case of the magnetic moments. We do not list them here because there are no experimental data available, instead we concentrate on the electromagnetic ratio of the $\Delta$ photo-decay amplitudes. For this purpose we add here still the quadrupole moments

$$
\begin{aligned}
Q^{\Delta^{++}} & =<\Delta^{++}, S_{3}=\frac{3}{2}|Q| \Delta^{++}, S_{3}=\frac{3}{2}>=-\frac{2}{25}<r^{2}>_{\Theta} \\
Q^{N \Delta} & =<\Delta^{+}, S_{3}=\frac{1}{2}|Q| p, S_{3}=\frac{1}{2}>=-\frac{\sqrt{2}}{30}<r^{2}>_{\Theta} .
\end{aligned}
$$


Our favorite models $A$ and $B$ give $Q^{\Delta^{++}} \simeq-0.06 \mathrm{fm}^{2}$, the transition moments are listed in table 3.3. Note in this context that the quadrupole moments are nonadiabatic quantities of $\mathcal{O}\left(N_{C}^{0}\right)$ which we calculate in tree approximation only.

\subsection{2 $E 2 / M 1$ ratio of the $\Delta$ photo-decay amplitudes}

The desire for precise values of the $\Delta$ photo-decay amplitudes has spawned numerous theoretical and experimental investigations in recent years. Much of this effort has been directed toward the $E 2 / M 1$ ratio of these amplitudes. In soliton models from the earliest attempts on [79 80 81 down to very recent publications [82 83 the $E 2$ transition was always calculated via the time component of the vector current $V_{0}^{3}$ by employing Siegert's theorem [84] at the finite photon momentum, where it is not strictly valid. We calculate the $E 2$ transition directly from the spatial components of the vector current $V_{i}^{3}$, show that indeed for $q \rightarrow 0$ the transition matrix element may be expressed by the corresponding matrix element of $V_{0}^{3}$ (Siegert's theorem), but we find nonnegligible corrections at the photon point leading to a noticeable reduction of the electromagnetic ratio.

For this purpose we have to solve the e.o.m.

$$
-\partial^{i} V_{i}^{a}=\partial_{i} V_{i}^{a}=\dot{V}_{0}^{a}=\frac{f_{\pi}^{2}}{\Theta^{2}} s^{2} b_{T} D_{a p}(\hat{\boldsymbol{r}} \times \boldsymbol{R})_{p}(\hat{\boldsymbol{r}} \boldsymbol{R})
$$

to $\mathcal{O}\left(N_{C}^{-1}\right)$ because the $E 2$ transition is supressed by $1 / N_{C}^{2}$ as compared to the $M 1$ transition. This leads to small rotationally induced monopole and quadrupole deformations

$$
\begin{aligned}
\eta_{L}^{\Omega} & =\frac{1}{6 f_{\pi} \Theta^{2}}\left[2 f(r) \boldsymbol{R}^{2}+u(r)\left(\boldsymbol{R}^{2}-3(\hat{\boldsymbol{r}} \boldsymbol{R})^{2}\right)\right] \\
\boldsymbol{\eta}_{T}^{\Omega} & =-\frac{1}{2 f_{\pi} \Theta^{2}} v(r)(\boldsymbol{R}-\hat{\boldsymbol{r}}(\hat{\boldsymbol{r}} \boldsymbol{R}))(\hat{\boldsymbol{r}} \boldsymbol{R})
\end{aligned}
$$

described in appendix A.1. The system of differential equations for the radial functions $f(r), u(r)$ and $v(r)$ is identical to the one discussed for the magnetic polarizabilities (subsection 3.5.4), and consequently also the radial functions shown in fig. 3.5. With these components inserted, the vector current obeys (3.118). For this reason we will not encounter ambiguous results calculating the $E 2$ transition via the time or the spatial components of the electromagnetic current, discussed for constituent quark models in 85.

In order to minimize errors, the so-called "equal-velocity" frame where the incoming nucleon and the outgoing $\Delta$ have opposite velocities and which 
reduces to the Breit frame as the nucleon- $\Delta$ split vanishes, is chosen as a convenient reference frame [86. In this frame the photon momentum is $q_{\Delta}=$ $\left(M_{\Delta}-M_{N}\right) / 2 \sqrt{M_{\Delta} M_{N}}=296 \mathrm{MeV}$. We define the electromagnetic ratio by the ratio of the corresponding helicity amplitudes

$$
\frac{E 2}{M 1}=\frac{1}{3} \frac{A_{\frac{1}{2}}(E 2)}{A_{\frac{1}{2}}(M 1)}=\frac{1}{3} \frac{<\Delta^{+}, S_{3}=\frac{1}{2}\left|M_{\lambda=1}^{E 2}\right| p, S_{3}=-\frac{1}{2}>}{<\Delta^{+}, S_{3}=\frac{1}{2}\left|M_{\lambda=1}^{M 1}\right| p, S_{3}=-\frac{1}{2}>}
$$

with the multipole operators 87]

$$
\begin{array}{r}
M_{\lambda}^{M 1}\left(q^{2}\right)=i \sqrt{6 \pi} \lambda \int d^{3} r V_{i}^{3} j_{1}(q r)\left[\boldsymbol{Y}_{11 \lambda}(\hat{\boldsymbol{r}})\right]_{i} \\
M_{\lambda}^{E 2}\left(q^{2}\right)=\frac{\sqrt{10 \pi}}{q} \int d^{3} r V_{i}^{3}\left[\boldsymbol{\nabla} \times\left(j_{2}(q r) \boldsymbol{Y}_{22 \lambda}(\hat{\boldsymbol{r}})\right)\right]_{i} .
\end{array}
$$

First we are going to evaluate the $M 1$ transition operator by inserting the vector current in tree +1 -loop (which part may be expressed by the isovector magnetic formfactor discussed in section 3.4) as well as the rotationally induced soliton deformations of $\mathcal{O}\left(N_{C}^{-1}\right)$

$$
\begin{aligned}
M_{\lambda}^{M 1}\left(q^{2}\right)=-\frac{3 \lambda}{2} & {\left[\frac{q}{M_{N}} G_{M}^{V}\left(q^{2}\right) D_{3 \lambda}\right.} \\
& \left.+2 f_{\pi} D_{3 p} \int d^{3} r j_{1} \frac{s}{r}\left(c\left(\delta_{p \lambda}-\hat{r}_{p} \hat{r}_{\lambda}\right) \eta_{L}-\hat{r}_{p} \eta_{T \lambda}+\cdots\right)\right] \\
=-\frac{3 \lambda}{2} & {\left[\frac{q}{M_{N}} G_{M}^{V}\left(q^{2}\right) D_{3 \lambda}\right.} \\
& +\frac{1}{45 \Theta^{2}} \int d^{3} r j_{1} \frac{s}{r}(20 c f-2 c u-3 v+\cdots) \frac{1}{2}\left\{D_{3 \lambda}, \boldsymbol{R}^{2}\right\} \\
& \left.+\frac{1}{15 \Theta^{2}} \int d^{3} r j_{1} \frac{s}{r}(2 c u+3 v+\cdots) L_{3} R_{\lambda}\right] .
\end{aligned}
$$

With this multipole operator, the $M 1$ transition matrix element in tree + 1-loop with soliton deformations included

$$
\begin{aligned}
& <\Delta^{+}, S_{3}=\frac{1}{2}\left|M_{\lambda=1}^{M 1}\right| p, S_{3}=-\frac{1}{2}>=-\frac{q}{2 \sqrt{2} M_{N}} G_{M 1}^{N \Delta}\left(q^{2}\right) \\
& G_{M 1}^{N \Delta}\left(q^{2}\right)=\sqrt{2}\left\{G_{M}^{V}\left(q^{2}\right)+\frac{M_{N}}{20 q \Theta^{2}} \int d^{3} r j_{1} \frac{s}{r}[2 c(10 f-u)-3 v+\cdots]\right\} .
\end{aligned}
$$

is determined.

For the E2 transition there is no choice: the soliton deformations (3.119) must be inserted, otherwise the operator vanishes. Alternatively, and of course with the same result, we may employ partial integration and the vector 
current conservation (3.118)

$$
\begin{aligned}
& M_{\lambda}^{E 2}\left(q^{2}\right)=\frac{1}{i q} \sqrt{\frac{5 \pi}{3}} \int d^{3} r {\left[\left(3 j_{2}-q r j_{3}\right) \partial_{i} V_{i}^{3}-q^{2} j_{2} x_{i} V_{i}^{3}\right] Y_{2 \lambda} } \\
&=\frac{1}{i q} \sqrt{\frac{5 \pi}{3}} \int d^{3} r {\left[\left(3 j_{2}-q r j_{3}\right) \dot{V}_{0}^{3}\right.} \\
&\left.+f_{\pi} q^{2} j_{2} D_{3 p}\left(r F^{\prime} c \hat{\boldsymbol{r}} \times \boldsymbol{\eta}-r s \hat{\boldsymbol{r}} \times \boldsymbol{\eta}^{\prime}+\cdots\right)_{p}\right] Y_{2 \lambda} \\
&=\sqrt{\frac{5 \pi}{3}} \frac{1}{\Theta} \int d^{3} r\left[f_{\pi}^{2}\left(3 j_{2}-q r j_{3}\right) s^{2} b_{T}-\frac{q^{2}}{2} j_{2}\left(r F^{\prime} c v-r s v^{\prime}+\cdots\right)\right] \\
& D_{3 p}\left(R_{p}-\hat{r}_{p}(\hat{\boldsymbol{r}} \boldsymbol{R})\right) Y_{2 \lambda} .
\end{aligned}
$$

In the last step the soliton deformations (3.119) (only $N \ell \sigma$ contributions are written out in explicit form) are inserted, and it is then noticed that the entire operator may be written as a total time derivative which in the end may be replaced by iq (compare the derivation of Siegert's theorem). The E2 transition matrix element is now

$$
\begin{gathered}
<\Delta^{+}, S_{3}=\frac{1}{2}\left|M_{\lambda=1}^{E 2}\right| p, S_{3}=-\frac{1}{2}>=-\frac{3 q^{2}}{4 \sqrt{2}} G_{E 2}^{N \Delta}\left(q^{2}\right) \\
G_{E 2}^{N \Delta}\left(q^{2}\right)=-\frac{\sqrt{2}}{9 q^{2} \Theta} \int d^{3} r\left[f_{\pi}^{2}\left(3 j_{2}-q r j_{3}\right) s^{2} b_{T}-\frac{q^{2} j_{2}}{2}\left(r F^{\prime} c v-r s v^{\prime}+\cdots\right)\right] .
\end{gathered}
$$

It should be remembered here that $G_{E 2}^{N \Delta}$ is a nonadiabatic quantity which we calculate in tree approximation only. There may possibly be nonadiabatic 1-loop contributions of the same order which to calculate we are not yet in a position.

For $q \rightarrow 0$ we obtain $G_{E 2}^{N \Delta} \rightarrow Q^{N \Delta}$ (3.117) which is indeed Siegert's theorem. At the photon point the corrections (second and third term in (3.125)) are sizeable, numerical values for the parameter combinations $A, B$, and $C$ are compared in table 3.3. The reason for $\left|G_{E 2}^{N \Delta}-Q^{N \Delta}\right|$ being larger than expected is connected with the fact that the induced rotational components (fig. 3.5) fall off quite slowly which corresponds to a large effective radius of these corrections. With (3.123, 3.125) the electromagnetic ratio (3.120) is determined as

$$
\frac{E 2}{M 1}=\frac{q_{\Delta} M}{2} \frac{G_{E 2}^{N \Delta}\left(q_{\Delta}^{2}\right)}{G_{M 1}^{N \Delta}\left(q_{\Delta}^{2}\right)} \rightarrow \frac{q_{\Delta} M}{2} \frac{G_{E 2}^{N \Delta}\left(\Delta^{2}\right)}{G_{M 1}^{N \Delta}\left(\Delta^{2}\right)} .
$$

In the last step a relativistic correction similar to (3.32) leads to the replacement $q_{\Delta} \rightarrow \Delta=M_{\Delta}-M_{N}=293 \mathrm{MeV}$ in the transition formfactors which numerically is of negligible influence. The results are listed in table 3.3 with an absolute value considerably smaller than in previous soliton calculations mainly for two reasons: 
- $G_{M 1}^{N \Delta}$ is larger and close to the experimental value at least for our favored models $A$ and $B$ (accidentally, maodel $A$ hits the experimental value of the corresponding helicity amplitude [78] exactly) because we included loop corrections of $\mathcal{O}\left(N_{C}^{0}\right)$ and soliton deformations of $\mathcal{O}\left(N_{C}^{-1}\right)$

- The consideration of $G_{E 2}^{N \Delta}\left(\Delta^{2}\right)$ instead of $Q^{N \Delta}\left(\Delta^{2}\right)$ (deviation from Siegert's theorem due to finite $\Delta$ ) reduces the absolute value of the electromagnetic ratio further.

Our result of $E 2 / M 1 \simeq-1.7 \%$ has to be compared with the experimental value reported by the particle data group [78] $-1.5 \pm 0.4 \%$ and a recent MAMI experiment with the preliminary result $-2.4 \pm 0.2 \%$ [88].

Table 3.3: Magnetic and quadrupole transition moments in units of nuclear magnetons and $\mathrm{fm}^{2}$ resp. are compared for the three parameter combinations $A, B$, $C$. The corresponding transition formfactors are evaluated at the photon point.

\begin{tabular}{|c|c|c|c|}
\hline & $A$ & $B$ & $C$ \\
\hline$\mu^{N \Delta}$ & 4.1 & 4.4 & 4.9 \\
$G_{M 1}^{N \Delta}\left(\Delta^{2}\right)$ & 3.2 & 3.4 & 3.8 \\
\hline$Q^{N \Delta}$ & -.034 & -.035 & -.038 \\
$Q^{N \Delta}\left(\Delta^{2}\right)$ & -.021 & -.022 & -.023 \\
$G_{E 2}^{N \Delta}\left(\Delta^{2}\right)$ & -.015 & -.015 & -.016 \\
\hline$\frac{E 2}{M 1}[\%]$ & -1.7 & -1.6 & -1.5 \\
\hline
\end{tabular}

Because the extraction of the electromagnetic ratio from experimental data is far from being trivial [89] [90], a complete calculation of the $\gamma N \rightarrow \pi N$ reaction, which includes the background with the $\Delta$ resonance lying in the continuum would be desirable in order to obtain complex helicity amplitudes for the $N \Delta$ transition. Although such a calculation should in principle be feasible within soliton models, the effort would be tremendeous. 


\section{Chapter 4}

\section{Results}

In this chapter, we are finally going to present and discuss results obtained by applying the method outlined above to the quantities introduced in the preceding paragraphs. As a shorthand, we shall in the following denote quantities whose loop corrections can be treated in adiabatic approximation (and are therefore calculated in this paper) as adiabatic quantities, whereas the others (for which we are unable to calculate the loop correction) will be summarized as nonadiabatic ones; only their tree level values will be given. For all quantities considered, we evaluate the Lagrangian (2.47) for the three parameter sets $A\left(e=4.25, g_{\omega}=0\right), B\left(e=4.5, g_{\omega}=1.0\right)$ and $C\left(e=5.845, g_{\omega}=2.2\right)$ introduced in section 2.2 . From the remarks in that section, it should be clear that we cannot expect model $C$ to result in sensible numbers since the local approximation with vector meson parameters overestimates the effect from the $\omega$ meson and neglects important contributions of higher chiral orders. However, sets $A$ and $B$, designed to simulate these missing pieces by introducing 'effective' parameter values should give an accurate picture of the abilities (and defects) of the soliton approach with respect to the quantities under investigation. Since amongst these three, set $B$ comes closest to the original vector meson model, we consider this to be the most realistic one.

Before entering the discussion of magnitude and sign of the 1 loop corrections it is worthwhile to recall the salient features of the tree approximation within the three parameter sets $A, B$ and $C$, at least for the adiabatic quantities:

- The soliton mass has been a constant source of embarrassment for the past decade, since it came out too large by a factor of more than $3 / 2$ almost regardless of parameter combinations used or meson species included in the model. This is also obvious from tables 4.1, 4.2 and 4.3, where the tree level mass is shown to be in the $1500-1630 \mathrm{MeV}$ range 
for the three models.

- With values between 54 and $67 \mathrm{MeV}$ against experimental $45 \mathrm{MeV}$, the $\boldsymbol{\sigma}$ term is also overestimated in tree for all parameter combinations under consideration here. This is not a common feature of all Skyrme type models and depends on our usage of the full $\mathrm{ChO} 4$ lagrangian including symmetry breakers. The effect is much less pronounced than in case of the mass, especially if the comparably large error margin of the experimental datum is taken into account. Nevertheless, a sizeable reduction due to 1 loop corrections would be necessary. Partly due to the overestimated $\sigma$ term, the scalar radius comes out consistently much too low in tree, its value of around $1 \mathrm{fm}$ as opposed to experimental $1.6 \mathrm{fm}$ being rather insensitive to the parameters used.

- The axial vector constant is a weak point of Skyrme type models, since the tree contribution doggedly refuses to grow appreciably beyond 1.0 (our values being .91-1.03) as long as reasonable parameters are considered, where reasonable means that the other quantities should not be completely off the mark. Likewise, the quantity $g_{A}\left\langle r^{2}\right\rangle_{A}$ is underpredicted in all cases, numerical values ranging from $.36 \mathrm{fm}^{2}$ to $.42 \mathrm{fm}^{2}$ whereas experiment points to $.53 \mathrm{fm}^{2}$.

- Concerning the electromagnetic formfactors, it has long been recognized that magnetic moments generally come out too small, most notably the (nonadiabatic) isoscalar one, which is wrong by a factor of 2 , but also the isovector moment for which our parameters give $1.6-1.8$ nuclear magnetons in contrast to the experimental finding of 2.35. Similarly, for some time, conventional wisdom had it that e.m. radii were seriously underpredicted in purely pionic models. However, inclusion of nonminimal couplings with coupling constants chosen in accordance with the logic presented in section 2.2, as well as taking into account relativistic corrections serve to remedy this problem already at tree level, and for our parameter sets, actually the converse is true: isoscalar electric as well as isovector magnetic radius are slightly overestimated with values of $.61-.67 \mathrm{fm}^{2}$ for the former and $.77-.72 \mathrm{fm}^{2}$ for the latter case to be compared to experimental data of $.59 \mathrm{fm}^{2}$ and $.73 \mathrm{fm}^{2}$, respectively.

- The electric polarizability is consistently too large in tree without regard of the model considered; the discussion in the corresponding section of the previous chapter has revealed that this would also hold in models containing vector mesons explicitly, which were for some time believed to cure that deficiency. Actual numbers are in the $18-22$. 
$10^{-4} \mathrm{fm}^{3}$ bracket for our models; however, the amount by which the polarizability is overestimated is difficult to quantify in view of the large error bar, the experimental value being $9.5 \pm 5 \cdot 10^{-4} \mathrm{fm}^{3}$.

Turning now to the loop corrections, inspection of tables 4.1, 4.2 and 4.3 provides a rough classification in terms of the sensitivity to the stabilisation mechanism: quantities with a loop contribution depending sensitively on the choice of parameters comprise $\sigma$ term, $g_{A}$, axial radius end electric polarizability. All others are rather insensitive to the details of the lagrangian. We shall postpone discussion of the first kind of properties and deal firstly with the latter species. Here, the most pronounced results are the following:

- As had been shown previously (36] 35]) the soliton mass is considerably reduced towards its experimental value once loop corrections are taken into account. Every parameter set considered here results in a tree +1 loop mass of around $950 \mathrm{MeV}$.

- No less importantly, the scalar radius is considerably enhanced towards the experimental datum, with tree +1 loop results in the range of $1.3-1.5 \mathrm{fm}^{2}$.

- The same is true for the isovector magnetic moment, which shows an enhancement to $2.24-3.13$. The latter number (3.13 for set $C$ ) actually reverses the tree level underprediction into a considerable overestimation, but both the other models are satisfactory.

In all these cases, the quantum correction has the right sign and a magnitude of slightly more than $1 / 3$ relative to the tree level value.This serves as an impressive justification of the conjecture that loop corrections might play a crucial role in the understanding of baryon properties in soliton models.

Less marked changes occur in the e.m. radii, where the correction is around $1 / 6$ of tree, yet has the right sign in all cases except $C$, where the too small tree magnetic radius is further reduced. Since the tree level values were already rather good, it is not really justified to speak of an improvement here; however, the fact that loop contributions are tiny where one would expect them to be, and generally have, once again, the right sign supports the hypothesis that quantum corections, properly taken into account, could eventually cure the defects of tree level evaluated Skyrme type models without worsening things with respect to quantities where tree already gives acceptable results.

So far, there has been little to choose between sets $A, B$ and $C$ with the exception of magnetic properties in case $C$. This radically changes if one 
considers the first kind of quantities mentioned above, namely $\sigma$ term, $g_{A}$, axial radius and electric polarizability.

Dealing first with the $\boldsymbol{\sigma}$ term, this happens to be corrected in the right direction and with the right magnitude for the first two parameter sets $A$ and $B$; actually, set $A$ turns the too large tree value into a too small tree + 1 loop one $(32 \mathrm{MeV})$, whereas set $B$ is just about right $(44 \mathrm{MeV})$. However, the startling observation is that for a strong enough sixth order term $(\operatorname{set} C$ ) the 1-loop contribution changes sign and increases the already too large tree value to about $80 \mathrm{MeV}$. A similar phenomenon occurs in case of the $\boldsymbol{g}_{A}$ if one takes into account set $D$, and also for the axial radius. We do not want to repeat the discussion of section 3.3 here, but once again we emphasise that the changing sign of the loop correction serves as a filter to decide which model to discard and which to keep; sets $C$ and $D$ are clearly ruled out by the numbers. (Note that in contrast to the situation for the slope of the axial formfactor $g_{A}<r^{2}>_{A}$, the 1 loop correction to the radius itself is not worrisome, and that $\left\langle r^{2}\right\rangle_{A}$ comes out too large, yet within the error margin of the experimental datum, if one includes the estimated $1 / N_{C}$ piece). The fairly good stability of the tree +1 loop value of $g_{A}$ over a wide range of scale as shown in fig. 3.3 probably rules out missing higher chiral orders to result in a scenario different from the one presented here (e.g., positive loop correction for otherwise reasonable parameter sets). Eventually, one of the success storys of our endeavour is the loop contribution to the electric polarizability, which turns out to bring both, models $A$ and $B$, to the experimental datum including error bars (tree +1 loop values being 9.8 and 14.1 respectively). Like its precursors discussed above, the correction changes sign as one proceeds from set $B$ to set $C$, inflating the tree value of 22 to about 27, therefore adding further to the list of its deficiencies. We believe this sensitivity to the strength of the sixth order term in the lagrangian to reflect the problems of the local approximation on the $\omega$ meson discussed above: A strong sixth order term gives rise to unphysical features in the scattering phaseshifts, and consequently fails to result in sensible predictions for certain quantities. Since we cannot, for the moment, properly account for the $\omega$ meson, we have to be content with the philosophy adopted in section 2.2 , namely to use effective values for $e$ and $g_{\omega}$ which then results in good agreement with experiment.

Summarising, we have shown loop corrections to bring all adiabatic quantities with the exception of the axial ones, close to their experimental values for parameter sets $A$ and $B$. In case of $g_{A}$ and $<r^{2}>_{A}$, we believe (section 3.3) that their status is exceptional and that the $1 / N_{C}$ expansion must run into difficulties in their case. We have provided an estimate of the next- tonext-to-leading order correction to them, which turned out to be large and 
Table 4.1: Tree and 1-loop contribution to various quantities for parameter set $A\left(e=4.25, g_{\omega}=0\right)$.

\begin{tabular}{|c|c|c|c|c|}
\hline & tree & 1-loop & $\sum$ & exp. \\
\hline$M[\mathrm{MeV}]$ & 1629 & -683 & 946.0 & 939 \\
\hline$\sigma[\mathrm{MeV}]$ & 54 & -22 & 32 & $45 \pm 7$ \\
\hline$<r^{2}>^{S}\left[\mathrm{fm}^{2}\right]$ & 1.0 & +.3 & 1.3 & $1.6 \pm .3$ \\
\hline$g_{A}$ & .91 & -.25 & .66 & 1.26 \\
\hline$<r^{2}>_{A}\left[\mathrm{fm}^{2}\right]$ & .45 & -.04 & .41 & $.42_{-.08}^{+.18}$ \\
\hline$<r^{2}>_{E}^{S}\left[\mathrm{fm}^{2}\right]$ & .62 & -.11 & .51 & .59 \\
\hline$\mu^{V}$ & 1.62 & +.62 & 2.24 & 2.35 \\
\hline$<r^{2}>_{M}^{V}\left[\mathrm{fm}^{2}\right]$ & .77 & -.13 & .64 & .73 \\
\hline$\alpha\left[10^{-4} \mathrm{fm}^{3}\right]$ & 17.8 & -8.0 & 9.8 & $9.5 \pm 5$ \\
\hline
\end{tabular}

Table 4.2: Tree and 1-loop contribution to various quantities for parameter set $B\left(e=4.5, g_{\omega}=1.0\right)$.

\begin{tabular}{|c|c|c|c|c|}
\hline & tree & 1-loop & $\sum$ & exp. \\
\hline$M[\mathrm{MeV}]$ & 1599 & -646 & 953 & 939 \\
\hline$\sigma[\mathrm{MeV}]$ & 58 & -14 & 44 & $45 \pm 7$ \\
\hline$<r^{2}>^{S}\left[\mathrm{fm}^{2}\right]$ & 1.1 & +.3 & 1.4 & $1.6 \pm .3$ \\
\hline$g_{A}$ & .96 & -.15 & .81 & 1.26 \\
\hline$<r^{2}>_{A}\left[\mathrm{fm}^{2}\right]$ & .44 & -.01 & .43 & $.42_{-.08}^{+.18}$ \\
\hline$<r^{2}>_{E}^{S}\left[\mathrm{fm}^{2}\right]$ & .64 & -.09 & .55 & .59 \\
\hline$\mu^{V}$ & 1.69 & +.88 & 2.57 & 2.35 \\
\hline$<r^{2}>_{M}^{V}\left[\mathrm{fm}^{2}\right]$ & .76 & -.17 & .59 & .73 \\
\hline$\alpha\left[10^{-4} \mathrm{fm}^{3}\right]$ & 19.4 & -5.3 & 14.1 & $9.5 \pm 5$ \\
\hline
\end{tabular}

Table 4.3: Tree and 1-loop contribution to various quantities for parameter set $C\left(e=5.845, g_{\omega}=2.2\right)$.

\begin{tabular}{|c|c|c|c|c|}
\hline & tree & 1-loop & $\sum$ & exp. \\
\hline$M[\mathrm{MeV}]$ & 1490 & -539 & 951 & 939 \\
\hline$\sigma[\mathrm{MeV}]$ & 67 & +13 & 80 & $45 \pm 7$ \\
\hline$<r^{2}>^{S}\left[\mathrm{fm}^{2}\right]$ & 1.1 & +.4 & 1.5 & $1.6 \pm .3$ \\
\hline$g_{A}$ & 1.03 & -.11 & .92 & 1.26 \\
\hline$<r^{2}>_{A}\left[\mathrm{fm}^{2}\right]$ & .40 & +.16 & .56 & $.42_{-.08}^{+.18}$ \\
\hline$<r^{2}>_{E}^{S}\left[\mathrm{fm}^{2}\right]$ & .68 & -.06 & .62 & .59 \\
\hline$\mu^{V}$ & 1.75 & +1.38 & 3.13 & 2.35 \\
\hline$<r^{2}>_{M}^{V}\left[\mathrm{fm}^{2}\right]$ & .72 & -.19 & .53 & .73 \\
\hline$\alpha\left[10^{-4} \mathrm{fm}^{3}\right]$ & 21.8 & +5.4 & 27.2 & $9.5 \pm 5$ \\
\hline
\end{tabular}


remedies the defects that show up including only $\mathcal{O}(1)$. Although there is in principle the possibility of sizeable $\mathcal{O}\left(N_{C}^{-1}\right)$ effects for the other quantities, too, already at this point, we can safely state that whatever parameter set (if any) might come out of a consideration including these must lie in the vicinity of $A$ or $B$ : $C$-like sets are clearly ruled out from the electric polarizability alone since they would require a $1 / N_{C}$ correction of around -10 as opposed to +5 at $\mathcal{O}(1)$. Table 4.5 gives tree results for nonadiabatic quantities. Here,

Table 4.4: Comparative listing of tree +1 loop values for all adiabatic quantities considered for the three parameter combinations $A, B, C$. Axial quantities include estimated $1 / N_{C}$ piece.

\begin{tabular}{|c|c|c|c|c|}
\hline & $A$ & $B$ & $C$ & exp. \\
\hline$M[\mathrm{MeV}]$ & 946 & 953 & 951 & 939 \\
\hline$\sigma[\mathrm{MeV}]$ & 32 & 44 & 80 & $45 \pm 7$ \\
\hline$<r^{2}>^{S}\left[\mathrm{fm}^{2}\right]$ & 1.3 & 1.4 & 1.5 & $1.6 \pm .3$ \\
\hline$g_{A}$ & 1.20 & 1.29 & 1.36 & 1.26 \\
\hline$<r^{2}>_{A}\left[\mathrm{fm}^{2}\right]$ & .55 & .54 & .59 & $.42_{-.08}^{+.18}$ \\
\hline$<r^{2}>_{E}^{S}\left[\mathrm{fm}^{2}\right]$ & .51 & .55 & .62 & .59 \\
\hline$\mu^{V}$ & 2.24 & 2.57 & 3.13 & 2.35 \\
\hline$<r^{2}>_{M}^{V}\left[\mathrm{fm}^{2}\right]$ & .64 & .59 & .53 & .73 \\
\hline$\alpha\left[10^{-4} \mathrm{fm}^{3}\right]$ & 9.8 & 14.1 & 27.2 & $9.5 \pm 5$ \\
\hline
\end{tabular}

Table 4.5: Comparative listing of tree values for all nonadiabatic quantities considered for the three parameter combinations $A, B, C$.

\begin{tabular}{|c|c|c|c|c|}
\hline & $A$ & $B$ & $C$ & exp. \\
\hline$\Delta[\mathrm{MeV}]$ & 290 & 278 & 265 & 293 \\
\hline$M_{n p}[\mathrm{MeV}]$ & 2.0 & 1.7 & 1.4 & $2.05 \pm .30$ \\
\hline$\left.<r^{2}\right\rangle_{E}^{V}\left[\mathrm{fm}^{2}\right]$ & .86 & .90 & .98 & $.85 \pm .03$ \\
\hline$\mu^{S}$ & .18 & .19 & .21 & .44 \\
\hline$<r^{2}>_{M}^{S}\left[\mathrm{fm}^{2}\right]$ & .74 & .75 & .77 & $.59 \pm .02$ \\
\hline$\triangle \alpha\left[10^{-4} \mathrm{fm}^{3}\right]$ & 1.7 & 1.8 & 1.9 & $5.0 \pm 5.0$ \\
\hline
\end{tabular}

one observes that all quantities except two are rather well reproduced already at this level. The two exceptions are the isoscalar magnetic moment and the 
neutron-proton-split of the electric polarizability. The latter is less severe in view of the huge error of the experimental value, but $\mu_{S}$ is seriously defective and it seems unlikely that a next order quantum correction would be able to remedy this discrepancy. In principle, however, there is the possibility of a quantum correction of the same order as the tree level in $1 / N_{C}$ since the time derivative contained in the spatial component of the winding number current expanded to quadratic order in the fluctuations may act on a fluctuation instead of a collective coordinate and twin fluctuations count as $1 / N_{C}$ like the angular velocity. We are not yet in a position to decide whether this is the root cause for the too small isoscalar magnetic moment.

Finally, we shall briefly recapitulate the course of this report: In the beginning we posed the question whether it is possible to renormalize a chiral lagrangian evaluated in a nontrivial topological sector in analogy to the methods known as chiral perturbatuion theory for the vacuum sector. The main problem is the restriction to a finite number of gradients contained in the lagrangian, which is not a priori justifiable in the presence of a soliton background. Upon considering a lagrangian incorporating resonance degrees of freedom we tried to better understand this issue and concluded that using the 4th order chiral lagrangian augmented by a standard 6th order extension including nonminimal couplings would be sufficient provided a shift away from the experimental values of $L E C$ s would be allowed for. Later on, we could add substance to this conclusion by considering the scale dependence of the tree +1 -loop soliton mass and the axial coupling constant $g_{A}$ whose values were shown to be remarkably stable over a wide range of the scale.

Starting from this lagrangian, we devised a method to calculate the self energy of the soliton in the presence of external fields in adiabatic approximation. In the course of the actual calculation, we had to clear several obstacles only indirectly related to our subject: We clarified the treatment of zero modes in the presence of external fields, showed how to properly compute the tree value of the electric polarizability and calculated the neutron-protonsplit of this quantity. In the end questions remain as to the convergence of the $1 / N_{C}$ expansion for axial quantities and to the size of the next-to-nextto-leading order contributions. However, we once again want to stress that in 7 out of 9 cases, the 1 loop correction has the right sign and magnitude to compensate for the discrepancy between tree level and experiment, which is truly remarkable since there are only two parameters $\left(e\right.$ and $\left.g_{\omega}\right)$ involved (which moreover have not been tuned to achieve some specific result).

The fact that sets $A$ and $B$ designed to simulate the higher chiral order pieces contained in a vector meson model indeed give much better results than set $C$ strongly supports models which include vector mesons explicitly. We decided to use a pseudoscalar lagrangian only because of the arguments 
presented in section 2.3. Nevertheless, we expect that, concerning 1-loop corrections, the results for a lagrangian with explicit $\varrho$ and $\omega$-mesons would come close to those of our model $B$. In particular the problems with the axial quantities discussed in section 3.3 will probably not be cured by the introduction of vector mesons.

\section{Acknowledgements}

The authors would like to express their gratitude for numerous stimulating discussions with their colleagues G. Holzwarth and B. Schwesinger. They are particularly indebted to B. Moussallam, who patiently taught them his method to calculate the Casimir energy in all details. 


\section{Bibliography}

[1] S. Weinberg, Physica 96A (1979) 327

[2] S. Weinberg, Phys. Lett. B251 (1990) 288

[3] S. Weinberg, Nucl. Phys. B363 (1991) 3

[4] J. Gasser and H. Leutwyler, Ann. Phys. 158 (1984) 142

[5] J. Gasser and H. Leutwyler, Nucl. Phys. B250 (1985) 465

[6] J. Gasser, M. E. Sainio and A. Svarc, Nucl. Phys. B307 (1988) 779

[7] E. Jenkins and A.V. Manohar, Phys. Lett. B255 (1991) 558

[8] T.H.R. Skyrme, Proc. Roy. Soc. A260 (1961) 127, Nucl. Phys. 31 (1962) 556

[9] G. t'Hooft, Nucl. Phys. B72 (1974) 461

[10] G. t'Hooft, Nucl. Phys. B75 (1974) 461

[11] E. Witten, Nucl. Phys. B160 (1979) 57

[12] G.S. Adkins, C.R. Nappi and E. Witten, Nucl. Phys. B228 (1983) 552

[13] G.S. Adkins and C.R. Nappi, Phys. Lett. B137 (1984) 251

[14] G. Holzwarth and B. Schwesinger, Rep. Prog. Phys. 49 (1986) 825 and references therein

[15] U.G. Meissner, N. Kaiser, A. Wirzba and W. Weise, Phys. Rev. Lett. 57 (1987) 1676

[16] I. Zahed and G.E. Brown, Phys. Rep. 142 (1987) 1

[17] J. Kunz, D. Masak and T. Reitz, Phys. Lett. B195 (1987) 459

[18] U.G. Meissner, Phys. Rep. 161 (1988) 213 and references therein 
[19] B. Schwesinger, H. Weigel, G. Holzwarth and A. Hayashi, Phys. Rep. 173 (1989) 173

[20] E.M. Nyman, Phys. Lett. B142 (1984) 388

[21] M. Chemtob, Nucl. Phys. A473 (1987) 613

[22] N.N. Scoccola and W. Weise, Phys. Lett. B232 (1989) 287

[23] N.N. Scoccola and W. Weise, Nucl. Phys. A517 (1990) 495

[24] S. Scherer and P.J. Mulders in "Proceedings of the international workshop on Baryons as Skyrme solitons", Siegen 1992, World Scientific, Singapore, Nucl. Phys. A549 (1992) 549

[25] B. Schwesinger in "Proceedings of the international workshop on Baryons as Skyrme solitons", Siegen 1992, World Scientific, Singapore

[26] B. Schwesinger, Phys. Lett. B298 (1993) 17

[27] J. Gasser, H. Leutwyler and M.E. Sainio, Phys. Lett. B253 (1991) 252

[28] A.V. Manohar, Phys. Lett. B336 (1994) 502

[29] N. Dorey and M.P. Mattis, hep-ph/9412373

[30] R. Rajaraman, Solitons and instantons, North Holland, Amsterdam 1982

[31] I. Zahed, A. Wirzba, U.G. Meissner, Phys. Rev. D33(1986) 830

[32] H. Schnitzer, Nucl. Phys. B261 (1985) 546

[33] A. Dobado and J. Terron, Phys. Lett. B247 (1990) 581

[34] B. Moussallam and D. Kalafatis, Phys. Lett. B272 (1991), 196

[35] G. Holzwarth in "Proceedings of the international workshop on Baryons as Skyrme solitons", Siegen 1992, World Scientific, Singapore, Phys. Lett. B291 (1992) 218

[36] B. Moussallam, Ann. Phys. 225 (1993) 264

[37] T.P. Cheng and L.F. Li, Gauge theory of elementary physics, Oxford University Press, Oxford 1985

[38] R.F. Dashen, B. Hasslacher and A. Neveu, Phys. Rev. D10 (1974) 4114, 4130

[39] H. Walliser and G. Eckart, Nucl. Phys. A429 (1984) 514 
[40] P.A.M. Dirac, Lectures on Quantum Mechanics, Belfer Graduate School of Science, 1964

[41] J. Schechter, Phys. Rev. D21 (1980) 3393

[42] H. Gomm, P. Jain, R. Johnson and J. Schechter, Phys. Rev. D33 (1986) 801,3476

[43] M.A. Shifmann, A.I. Vainstein and V.I. Zakharov, Nucl. Phys. B147 (1979) 385, 448

[44] P. Jain, R. Johnson, N.W. Park, J. Schechter and H. Weigel, Phys. Rev. D40 (1989) 855

[45] Ö. Kaymakcalan and J. Schechter, Phys. Rev. D31 (1985) 1109

[46] H. Walliser in "Proceedings of the international workshop on Baryons as Skyrme solitons", pp.247, Siegen 1992, World Scientific, Singapore

[47] G. Ecker, Czech. J. of Phys. 44 (1995) 405

[48] L.N. Epele et al., Phys. Rev. D39 (1989) 1473

[49] L.M. Barkov et al., Nucl. Phys. B256 (1985) 365

[50] G. Miller, B.M.K. Nefkins and I. Slaus, Phys. Rep. 194 (1990) 1

[51] M.P. Mattis and M. Karliner, Phys. Rev. D31 (1985) 2833

[52] F. Calogero, Variable Phase Approach to Potential Scattering, Acad. Press, New York and London, 1967

[53] T.N. Pham and T.N. Truong, Phys. Rev. D31 (1985) 3027

[54] J. Gasser, H. Leutwyler and M.E. Sainio, Phys. Lett. B253 (1991) 260

[55] V. Bernard, N.Kaiser, J. Kambor and U.G. Meissner, Nucl. Phys. B388 (1992) 315

[56] T.D. Cohen and W. Broniowski, Phys. Lett. B292 (1992) 5

[57] Xiandong Ji, Phys. Lett. B254 (1991) 456

[58] M. Kirchbach and D.O. Riska, Nuovo Cim. 104 (1991) 1837

[59] V. de Alfaro, S. Fubini, G. Furlan and C. Rossetti, Currents in Hadron Physics, Chapt.8, North Holland, Amsterdam 1973

[60] G. Dattoli, G. Matone and D. Prosperi, Lett. Nuovo Cim. 19 (1977) 601 
[61] D. Drechsel and A. Russo, Phys. Lett. 137 (1984) 294

[62] F. Schöberl and H. Leeb, Phys. Lett. 166 (1986) 355

[63] P.C. Hecking and G.F. Bertsch, Phys. Lett. B99 (1981) 237

[64] A. Schaefer et al., Phys. Lett. B143 (1984) 323

[65] R. Weiner and W. Weise, Phys. Lett. B159 (1985) 85

[66] V. Bernard, N. Kaiser and U.G. Meissner, Phys. Rev. Lett. 67 (1991) 1515

[67] V. Bernard, N. Kaiser and U.G. Meissner, Nucl. Phys. B373 (1992) 346

[68] F.J. Federspiel et. al., Phys. Rev. Lett. 47 (1991) 1511

[69] K.W. Rose et. al., Phys. Lett. B234 (1990) 460

[70] T.E.O. Ericson and J. Hüfner, Nucl. Phys. B57 (1973) 604

[71] J.L. Friar, Ann. Phys. 95 (1975) 170

[72] V.A. Petrun'kin, Sov. J. Part. Nucl. 12 (1981) 278

[73] J. Schmiedmayer et. al., Phys. Rev. Lett. 66 (1991) 1015

[74] W. Broniowski, M.K. Banerjee and T.D. Cohen, Phys. Lett. B283 (1992) 22

[75] A.I. L'vov, Int. J. of Mod. Phys. 48 (1993) 5267

[76] S. Saito and M. Uehara, Phys. Lett. B325 (1994) 20

[77] N.N. Scoccola and T.D. Cohen, preprint hep-ph/9507328

[78] Particle Data Group, Phys. Rev. D50 (1994) 1

[79] G.S. Adkins and C.R. Nappi, Nucl. Phys. B249 (1985) 507

[80] G.S. Adkins in: Chiral solitons, ed. K.F. Liu (World Scientific, Singapore, 1987) p.99

[81] A. Wirzba and W. Weise, Phys. Lett. B188 (1987) 6

[82] Ch.V. Christov et al., to be published in Prog. Nucl. and Part. Phys. (1996), ed. A. Faessler

[83] A. Abada, H. Weigel and H. Reinhardt, hep-ph/9509396 
[84] A.J.F. Siegert, Phys. Rev. 52 (1937) 787

[85] D. Drechsel and M.M. Giannini, Phys. Lett. B143 (1984) 329

[86] W. Warns, H. Schroeder, W. Pfeil, H. Rollnik, Z. Phys. C45 (1990) 627

[87] T.de Forest and J.D. Walecka, Adv. Phys. Vol.15 No.57 (1966) 1

[88] R. Beck, private communication, cited in [82

[89] R.M. Davidson, N.C. Mukhopadhyay and R.S. Wittman, Phys. Rev. D43 (1991) 71

[90] R.L. Workman, R.A. Arndt and Z. Li, Phys. Rev. C46 (1992) 1546

[91] N. Dorey, J. Hughes and M.P. Mattis, Phys. Rev. D50 (1994) 3874

[92] R. Oehme, Phys. Rev. 143 (1965) 1138

[93] B. Schwesinger and H. Walliser, Nucl. Phys. A490 (1988) 602 


\section{Appendix A}

\section{$1 / N_{C}$ contribution to $g_{A}$}

Although the relation (3.45) is model independent, the positivity of the remainder $R$ is not a direct implication of the charge algebra. Rather, this follows either from the experimental value of $g_{A}$ (which would be a circular argument in our context) or from the fact that $R$ can be expressed as a difference of pion nucleon cross sections which is experimentally known to be positive. In soliton models, it is hard to see whether $R$ is indeed positive independently of the parameter set chosen. Provided this were the case and the $1 / N_{C}$ expansion would converge for $R$, then $g_{A}$ would necessarily have to be larger than 1 as soon as the charge algebra relation (3.44) were fulfilled. We could then use a different strategy to obtain an estimate for the $1 / N_{C}$ piece of $g_{A}$, which in the end is equivalent to the naive addition of the CA "1" to $g_{A}^{2}$ used in section 3.3.3 .Such an estimate is the subject of this appendix.

Soliton models obey (3.44) provided nonadiabatic fluctuations which come with a collective angular velocity are taken into account [93]. Relative to the adiabatic fluctuations employed for the 1 loop contributions, these are suppressed by a factor of $1 / N_{C}$. As a consequence current algebra is reflected in $g_{A}$ not before $\mathcal{O}\left(N_{C}^{-1}\right)$ which, of course, is also immediately clear from (3.45).

Quite generally, the (spatially integrated) axial current up to $\mathcal{O}\left(N_{C}^{-1}\right)$ may be written as

$$
\mathcal{A}_{i}^{a}=-\frac{3}{2} g_{A}^{(1+0)} D_{a i}+\frac{1}{2} a^{(-1)}\left\{D_{a i}, \boldsymbol{R}^{2}\right\}+b^{(-1)} L_{a} R_{i}+c^{(-1)} D_{a i}
$$

where the $N_{C}$ orders are indicated by the superscripts.

In this object, the tree contribution proportional to the angular velocity squared of $\mathcal{O}\left(N_{C}^{-1}\right)$ due to the soliton's rotation can be shown to be negligible (subsection A.1). Adiabatic 2-loop diagrams cannot produce the operator 
structure of (A.1). There remain the nonadiabatic 1 loop processes: We believe that these should contribute sizeably in $\mathcal{O}\left(N_{C}^{-1}\right)$ to eq. (A.1) because exactly they are responsible for the restoration of the charge algebra commutation relation (3.44). However, they are too complicated to investigate in detail.

Therefore, in the second subsection of this appendix, in order to circumvent this obstacle, we are going to estimate the $1 / N_{C}$ contribution to $g_{A}$ produced by nonadiabatic 1 loop processes by imposing the $S U(4)$ current algebra commutation relations

$$
\left[\mathcal{A}_{3}^{a}, \mathcal{A}_{3}^{b}\right]=i \varepsilon_{a b c} L_{c}
$$

\section{A.1 Contribution due to rotationally induced soliton deformations}

Here we show that the effect of soliton deformations on the axial current is too weak to explain the relatively large $1 / N_{C}$ contributions needed in order to fulfill the CA commutation relation (A.2). This does of course not imply that soliton deformations are small by themselves, in the vector current they are responsible for sizeable contributions to the magnetic moments as we have noticed in subsection 3.6.2. Small deformations due to the soliton's rotation are driven by the term

$$
L_{\Omega}=f_{\pi} \int d^{3} r\left[s c\left(\hat{\boldsymbol{r}} \times \boldsymbol{\Omega}^{R}\right)^{2} \eta_{L}^{\Omega}-s\left(\hat{\boldsymbol{r}} \boldsymbol{\Omega}^{R}\right)\left(\boldsymbol{\Omega}^{R} \boldsymbol{\eta}_{T}^{\Omega}\right)+\cdots\right]
$$

in the lagrangian. The corresponding e.o.m.

$$
h_{a b}^{2} \eta_{b}^{\Omega}=f_{\pi}\left[s c \hat{r}_{a}\left(\hat{\boldsymbol{r}} \times \boldsymbol{\Omega}^{R}\right)^{2}-s\left(\Omega_{a}-\hat{r}_{a}\left(\hat{\boldsymbol{r}} \boldsymbol{\Omega}^{R}\right)\right)\left(\hat{\boldsymbol{r}} \Omega^{R}\right)+\cdots\right]
$$

may again be solved analytically in the asymptotical region

$$
\boldsymbol{\eta}^{\Omega \stackrel{r \rightarrow \infty}{=}} \frac{3 g_{A}}{16 \pi f_{\pi}} e^{-m_{\pi} r}\left[\hat{\boldsymbol{r}}\left(\boldsymbol{\Omega}^{R}\right)^{2}-\boldsymbol{\Omega}^{R}\left(\hat{\boldsymbol{r}} \boldsymbol{\Omega}^{R}\right)\right]
$$

which is in accordance with the result in ref. 91]. The full equations induce a monopole and a quadrupole deformation

$$
\begin{aligned}
\eta_{L}^{\Omega} & =\frac{1}{6 f_{\pi}}\left[2 f(r)\left(\boldsymbol{\Omega}^{R}\right)^{2}+u(r)\left(\left(\boldsymbol{\Omega}^{R}\right)^{2}-3\left(\hat{\boldsymbol{r}} \boldsymbol{\Omega}^{R}\right)^{2}\right)\right] \\
\boldsymbol{\eta}_{T}^{\Omega} & =-\frac{1}{2 f_{\pi}} v(r)\left(\boldsymbol{\Omega}^{R}-\hat{\boldsymbol{r}}\left(\hat{\boldsymbol{r}} \boldsymbol{\Omega}^{R}\right)\left(\hat{\boldsymbol{r}} \boldsymbol{\Omega}^{R}\right)\right)
\end{aligned}
$$


and it turns out that the system of differential equations for the radial functions $f(r), u(r)$ and $v(r)$ is identical to the ones discussed for the magnetic polarizabilities (subsection 3.5.4) and the electromagnetic ratio of the photodecay amplitudes (subsection 3.7.2). The radial functions are depicted in fig.3.5. These rotationally induced components may give rise to $1 / N_{C}$ contributions to the spatial axial current

$$
\begin{aligned}
\mathcal{A}_{i}^{a}= & \int d^{3} r x_{i}\left(\dot{A}_{0}^{a}-\partial^{\mu} A_{\mu}^{a}\right) \\
= & -\frac{3}{2} g_{A} D_{a i} \\
& +\frac{f_{\pi}^{2}}{2 \Theta^{2}} \int d^{3} r \operatorname{rsc} b_{T}\left(\frac{1}{2}\left\{D_{a i}, \boldsymbol{R}^{2}\right\}-L_{a} R_{i}\right) \\
& -\frac{m_{\pi}^{2}}{9 \Theta^{2}} \int d^{3} r \operatorname{rcf} \frac{1}{2}\left\{D_{a i}, \boldsymbol{R}^{2}\right\} \\
& -\frac{m_{\pi}^{2}}{15 \Theta^{2}} \int d^{3} r r\left(\frac{1}{3} c u+\frac{1}{2} v\right)\left(\frac{1}{2}\left\{D_{a i}, \boldsymbol{R}^{2}\right\}-3 L_{a} R_{i}\right) .
\end{aligned}
$$

Here we used partial integration and we listed only the contribution of the familiar symmetry breaking mass term. Equivalently, we could have inserted (A.6) directly into $\mathcal{A}_{i}^{a}$ with the same result.

From (A.7), we recover the operator structure (A.1) and we may read off the tree level pieces of the coefficients $a^{(-1)}$ and $b^{(-1)}$. Evaluating (A.7) in the chiral limit with the appropriate asymptotic expressions (3.114)

$$
\begin{aligned}
\mathcal{A}_{i}^{a}+\frac{3}{2} g_{A} D_{a i} \stackrel{m_{\underline{\pi}} \rightarrow 0}{=} & \frac{3 g_{A}}{2 \Theta^{2} m_{\pi}^{2}}\left(\frac{1}{2}\left\{D_{a i}, \boldsymbol{R}^{2}\right\}-L_{a} R_{i}\right) \\
& -\frac{g_{A}}{\Theta^{2} m_{\pi}^{2}} \frac{1}{2}\left\{D_{a i}, \boldsymbol{R}^{\mathbf{2}}\right\} \\
& -\frac{g_{A}}{2 \Theta^{2} m_{\pi}^{2}}\left(\frac{1}{2}\left\{D_{a i}, \boldsymbol{R}^{\mathbf{2}}\right\}-3 L_{a} R_{i}\right) \\
= & 0 \cdot \mathcal{O}\left(m_{\pi}^{-2}\right)
\end{aligned}
$$

the divergent terms cancel exactly which is pleasing because we expect the coefficients $a^{(-1)}$ and $b^{(-1)}$ to be finite in the chiral limit in order to fulfill the CA commutation relation( $(\widehat{A .2})$. According to this cancellation, the contributions $a^{(-1)}$ and $b^{(-1)}$ remain small also for finite pion mass. For example, model $A$ provides the tree contributions $a^{(-1)}=-.04$ and $b^{(-1)}=-.09$ which are negligible compared to the values $a^{(-1)}=-b^{(-1)}=.36$ necessary to fulfill the CA relation (A.2) (compare also the following subsection). Therefore we do not expect soliton deformations to appreciably contribute to this relation. 


\section{A.2 Contribution due to nonadiabatic loops}

In this section we estimate the $1 / N_{C}$ contribution to the axial current due to nonadiabatic loops by imposing the CA relation (A.2). Before doing so, we notice that in a pure Skyrme model (N $\ell \sigma$ model with $f_{\pi}$ and Skyrme term with parameter $e$ ) the coefficient in the axial current (A.1) scale according to

$$
g_{A}^{1} \sim \frac{1}{e^{2}}, \quad g_{A}^{0} \sim \frac{1}{f_{\pi}^{2} e^{2}}, \quad a^{(-1)} \sim b^{(-1)} \sim c^{(-1)} \sim \frac{1}{f_{\pi}^{2}}
$$

( $e=2 g$ is $\left.\mathcal{O}\left(N_{C}^{-1 / 2}\right)\right)$. Thus by choosing a large Skyrme parameter we can make the tree +1 loop contribution small. The property $g_{A} \geq 1$ must then almost entirely be achieved due to the $1 / N_{C}$ contribution.

The CA commutation relation (A.2) requires

$$
b^{(-1)}=-a^{(-1)}, \quad\left(a^{(-1)}\right)^{2}-\frac{4}{3}\left(g_{A}^{(1+0)}-\frac{2}{3} c^{(-1)}\right) a^{(-1)}+\frac{4}{9}=0
$$

and we may simplify the expression for the axial current (A.1)

$$
\mathcal{A}_{i}^{a}=-\frac{3}{2} g_{A}^{(1+0)} D_{a i}+a^{(-1)}\left[\frac{1}{2}\left\{D_{a i} \boldsymbol{R}^{2}\right\}-L_{a} R_{i}-\kappa D_{a i}\right],
$$

where we replaced $c^{(-1)}=-\kappa a^{(-1)}$ according to the scaling behaviour (A.9). The constant $\kappa$ is parameter independent and will be determined immediately. With (A.11) we may evaluate the weak axial nucleon-nucleon and nucleon- $\Delta$ transition amplitudes

$$
\begin{aligned}
g_{A} & =g_{A}^{(1+0)}+\frac{2}{3} \kappa a^{(-1)} \\
\frac{\sqrt{2}}{3} g_{A}^{*} & =g_{A}^{(1+0)}-\frac{3}{2} a^{(-1)}+\frac{2}{3} \kappa a^{(-1)} .
\end{aligned}
$$

With these quantities the condition (A.10) may be rewritten as

$$
\begin{aligned}
& g_{A}^{2}-\frac{2}{9} g_{A}^{* 2}=1 \\
& \frac{3}{2} a^{(-1)}=g_{A}-\frac{\sqrt{2}}{3} g_{A}^{*},
\end{aligned}
$$

which is Oehme's relation 92 and a direct consequence of the CA relation (A.2). From this relation it follows that $g_{A} \geq 1$. With the coefficient $a^{(-1)}$ inserted into (A.12) we obtain

$$
\begin{aligned}
g_{A} & =g_{A}^{(1+0)}+\frac{4}{9} \kappa\left(g_{A}-\frac{\sqrt{2}}{3} g_{A}^{*}\right) \\
& =g_{A}^{(1+0)}+\frac{4}{9} \kappa\left(g_{A}-\sqrt{g_{A}^{2}-1}\right)
\end{aligned}
$$


The last step involves the identification $\sqrt{2} / 3 g_{A}^{*}=+\sqrt{g_{A}^{2}-1}$ which is reasonable because of the large $N_{C}$ requirement $\sqrt{2} / 3 g_{A}^{*}=g_{A}$. Since relation A.14 must hold independently of the value of $g_{A}^{(1+0)}$ (c.f. the discussion in the beginning of this section), it follows that $\kappa \geq 9 / 4$. The choice $\kappa=9 / 4$ is conservative in the sense that it yields, for any given $g_{A}^{(1+0)}$, the smallest possible correction $g_{A}^{-1}$ (fig. A.1). Therefore, upon using this value for $\kappa$ we obtain

$$
\begin{aligned}
g_{A}^{2} & =1+\left(g_{A}^{(1+0)}\right)^{2} \\
\frac{\sqrt{2}}{3} g_{A}^{*} & =g_{A}^{(1+0)},
\end{aligned}
$$

which was the estimate used in section 3.3.3 .

Figure A.1: Plot of $g_{A}$ for different values of $g_{A}^{(1+0)}$.

From the experimental value $g_{A}=1.26$ we obtain a large $1 / N_{C}$ contribution $g_{A}^{(-1)}=.40$ and a small value $g_{A}^{(1+0)}=.86$ for tree +1 loop. If we accept the assumption underlying this paragraph, namely

- $S U(4)$ symmetry of the spatial axial current

then this seems to be the reason why soliton models in tree (+ 1 loop) approxmation always underestimate $g_{A}$ by a large margin. 


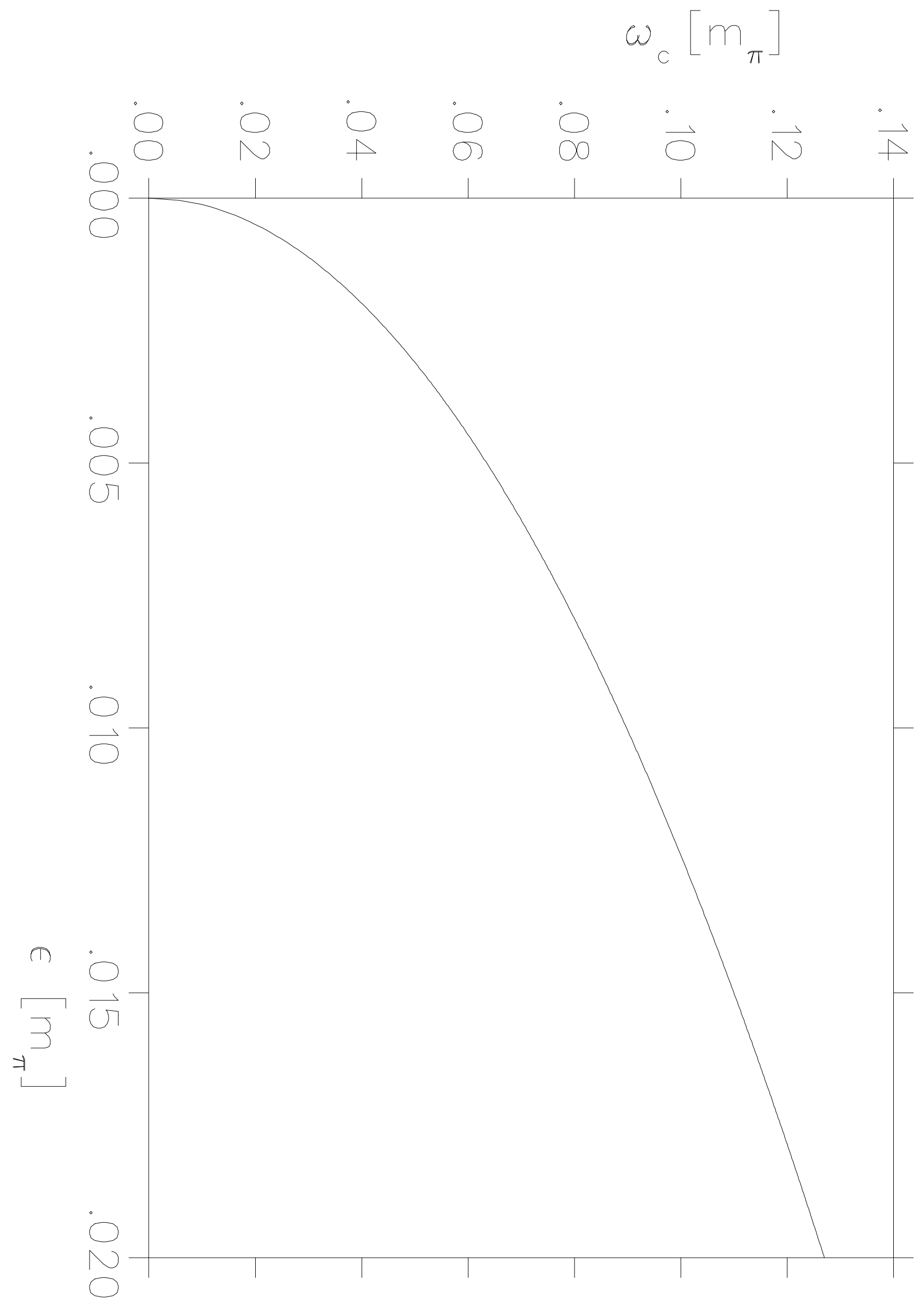




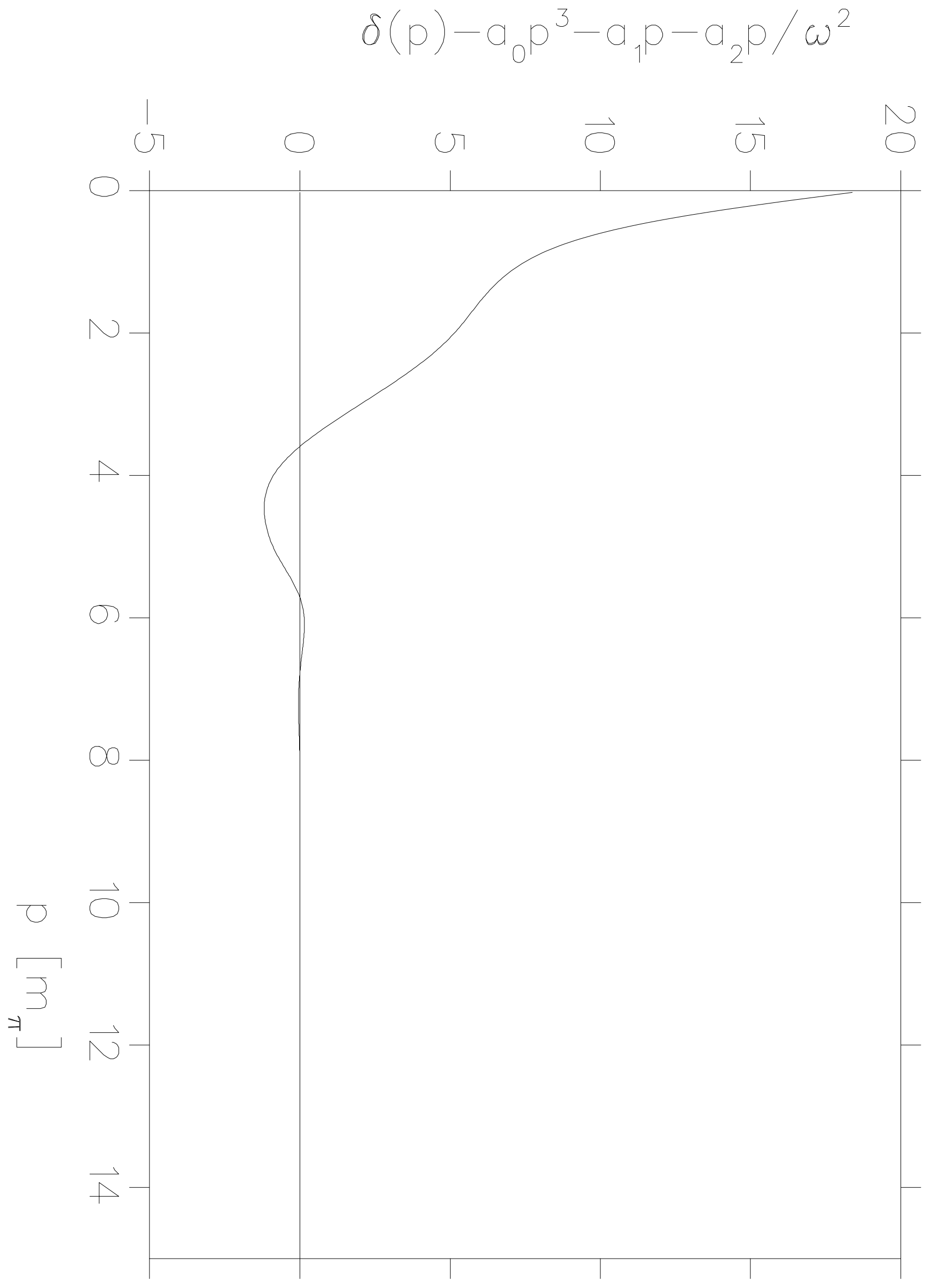




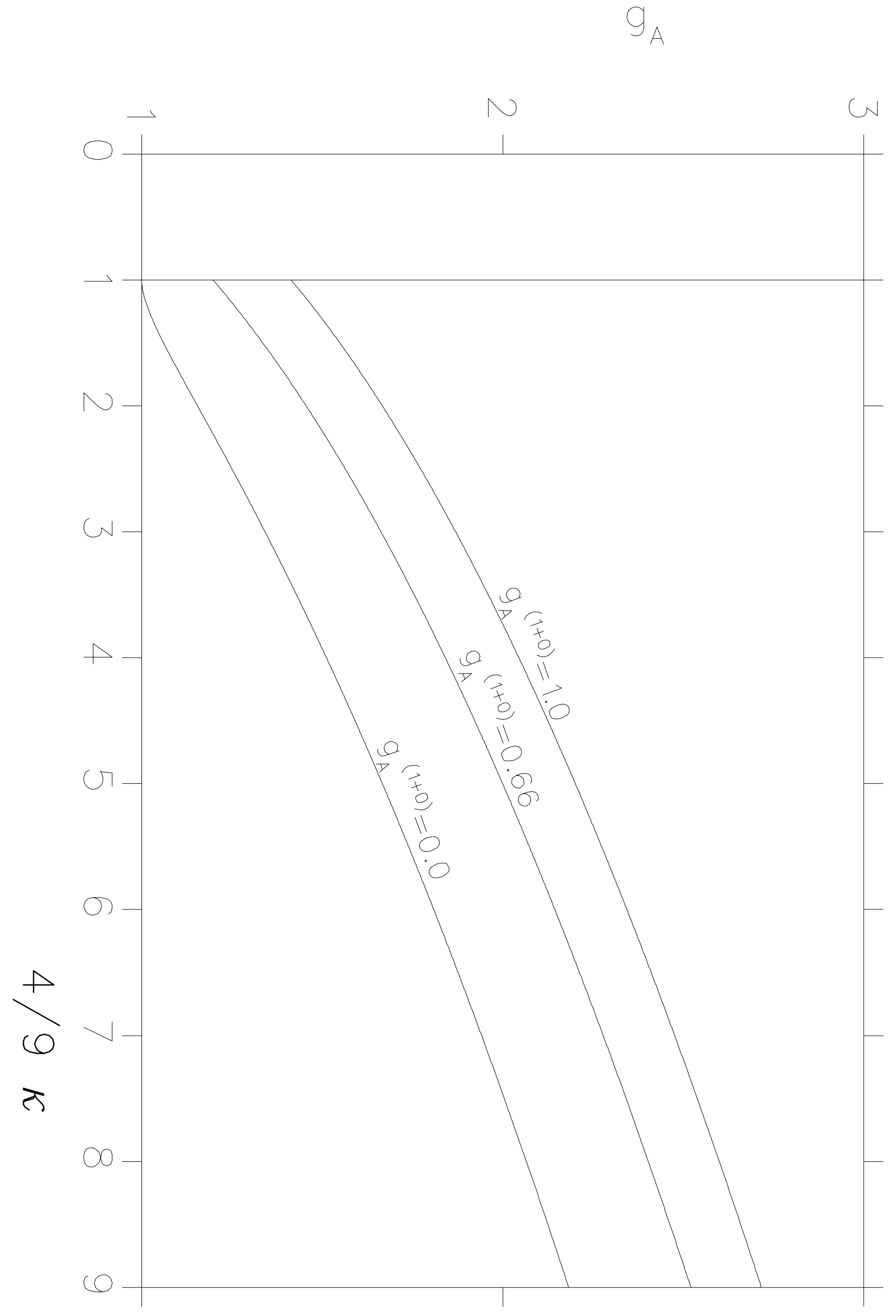




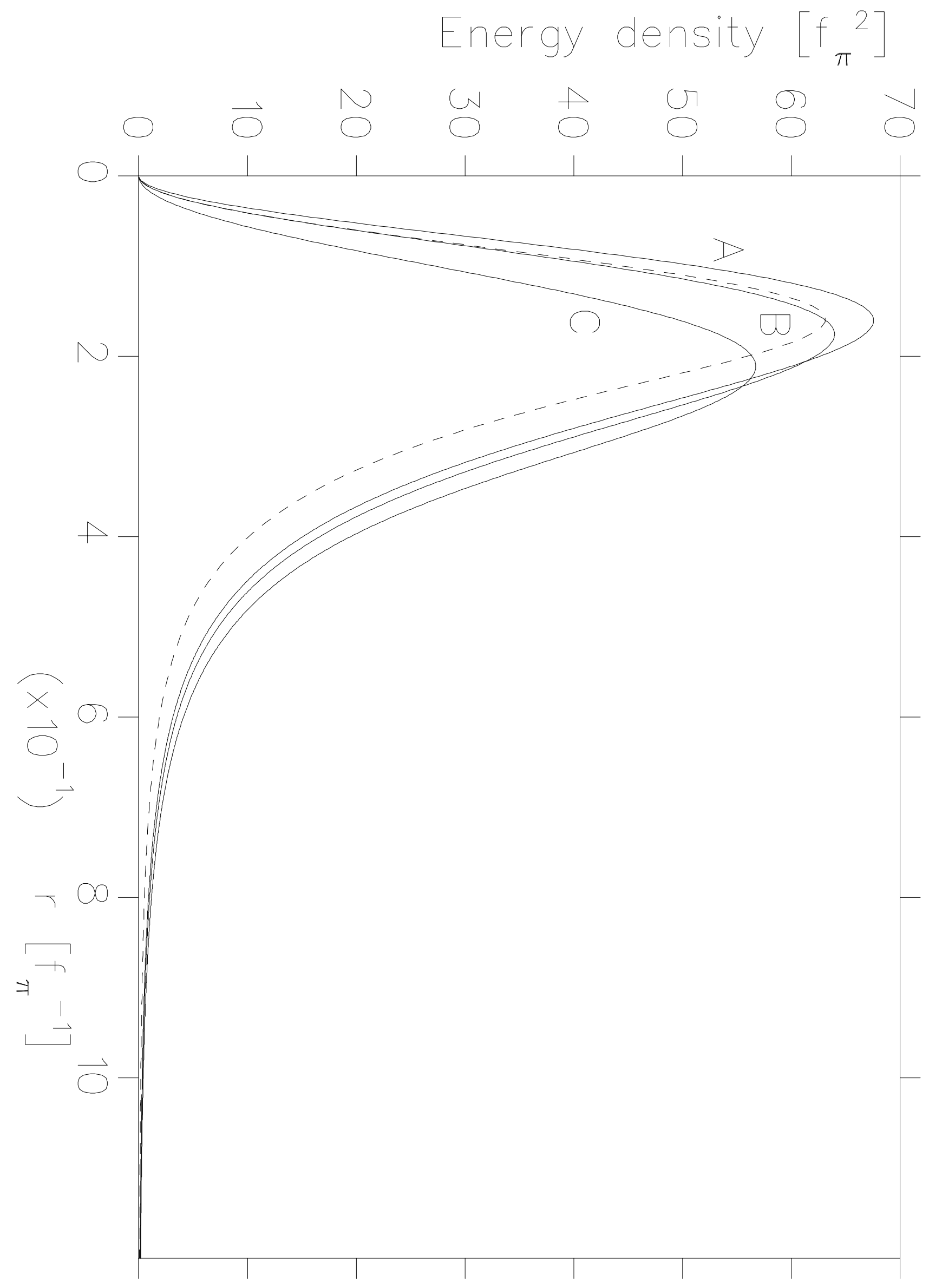




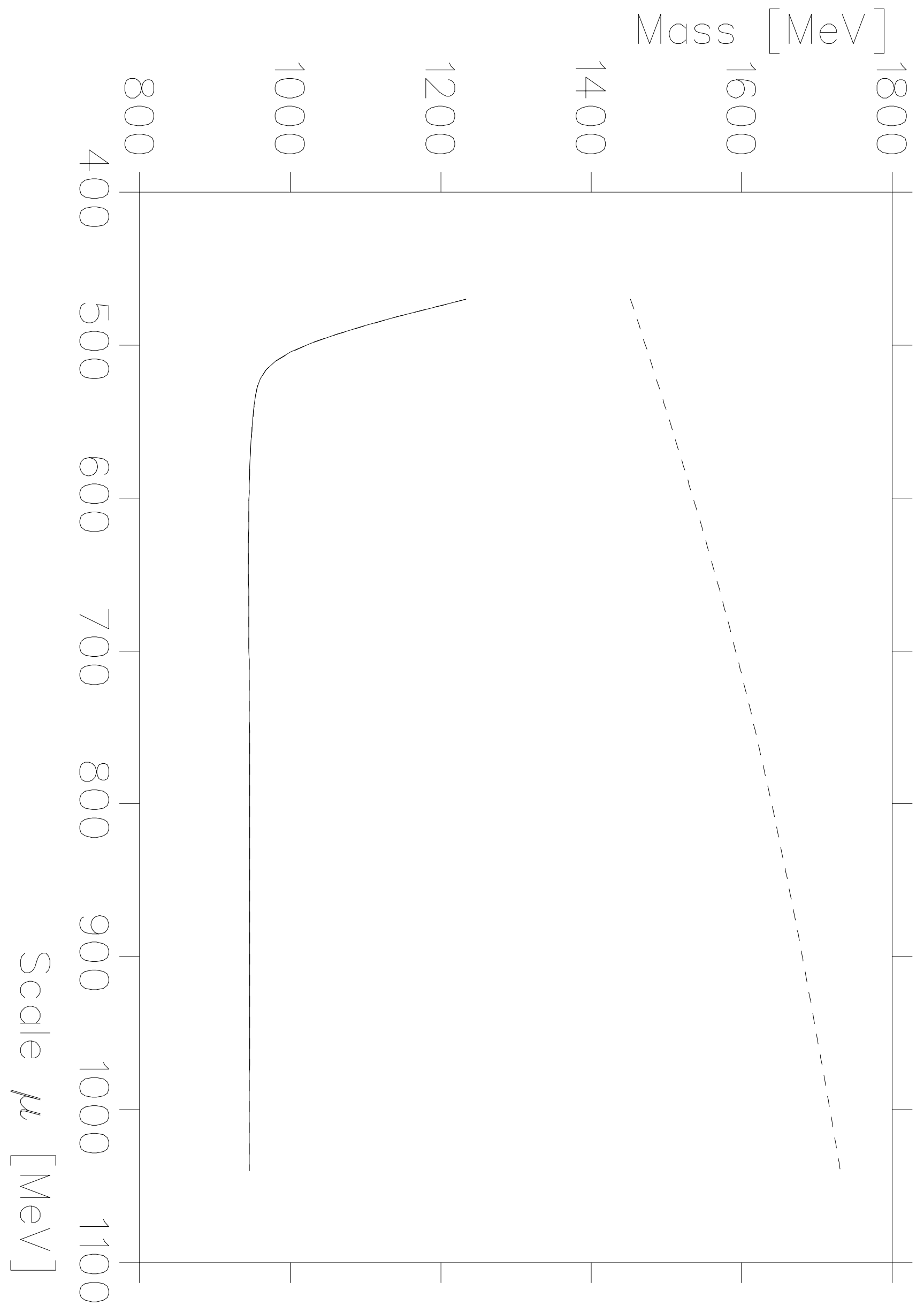




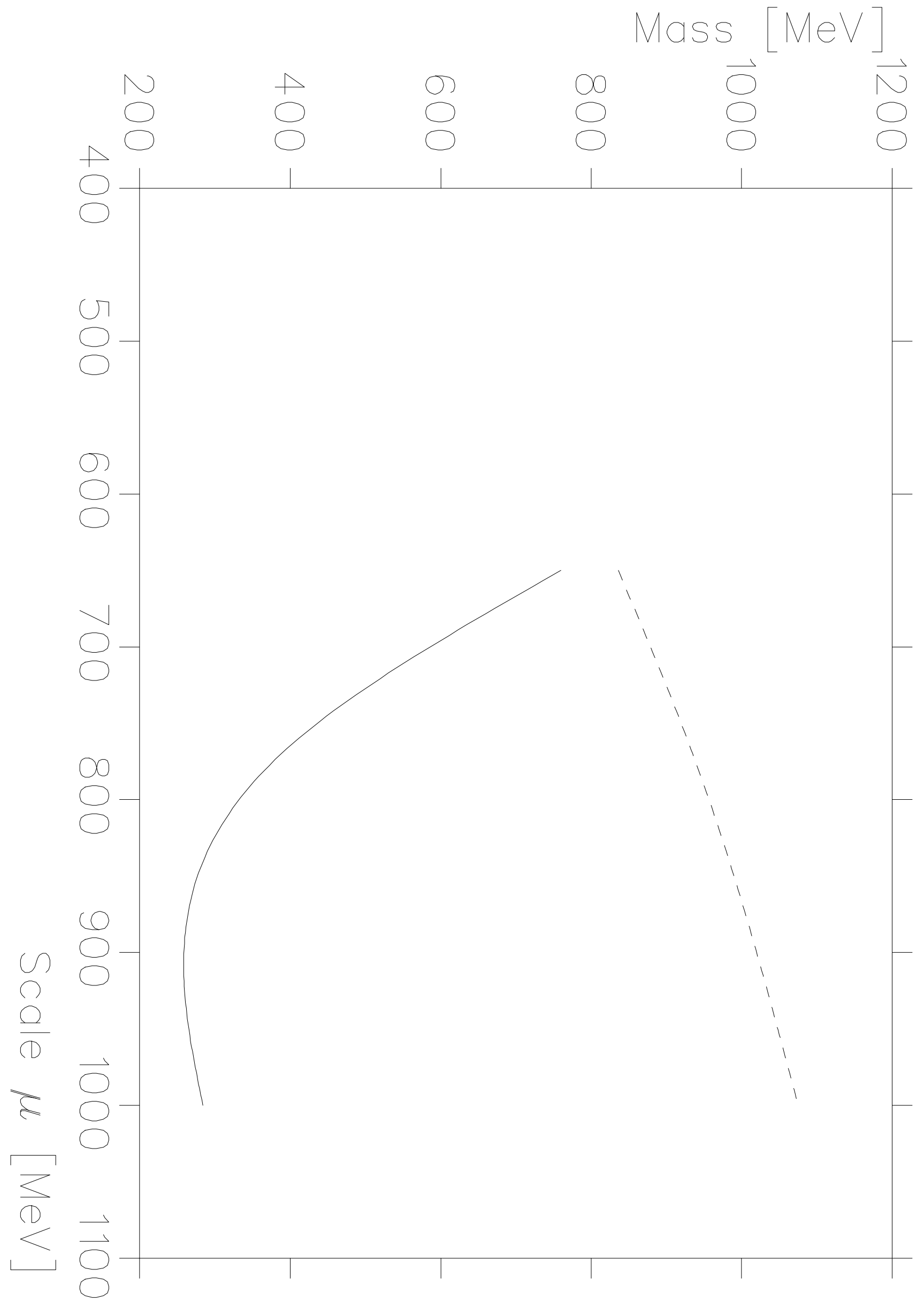




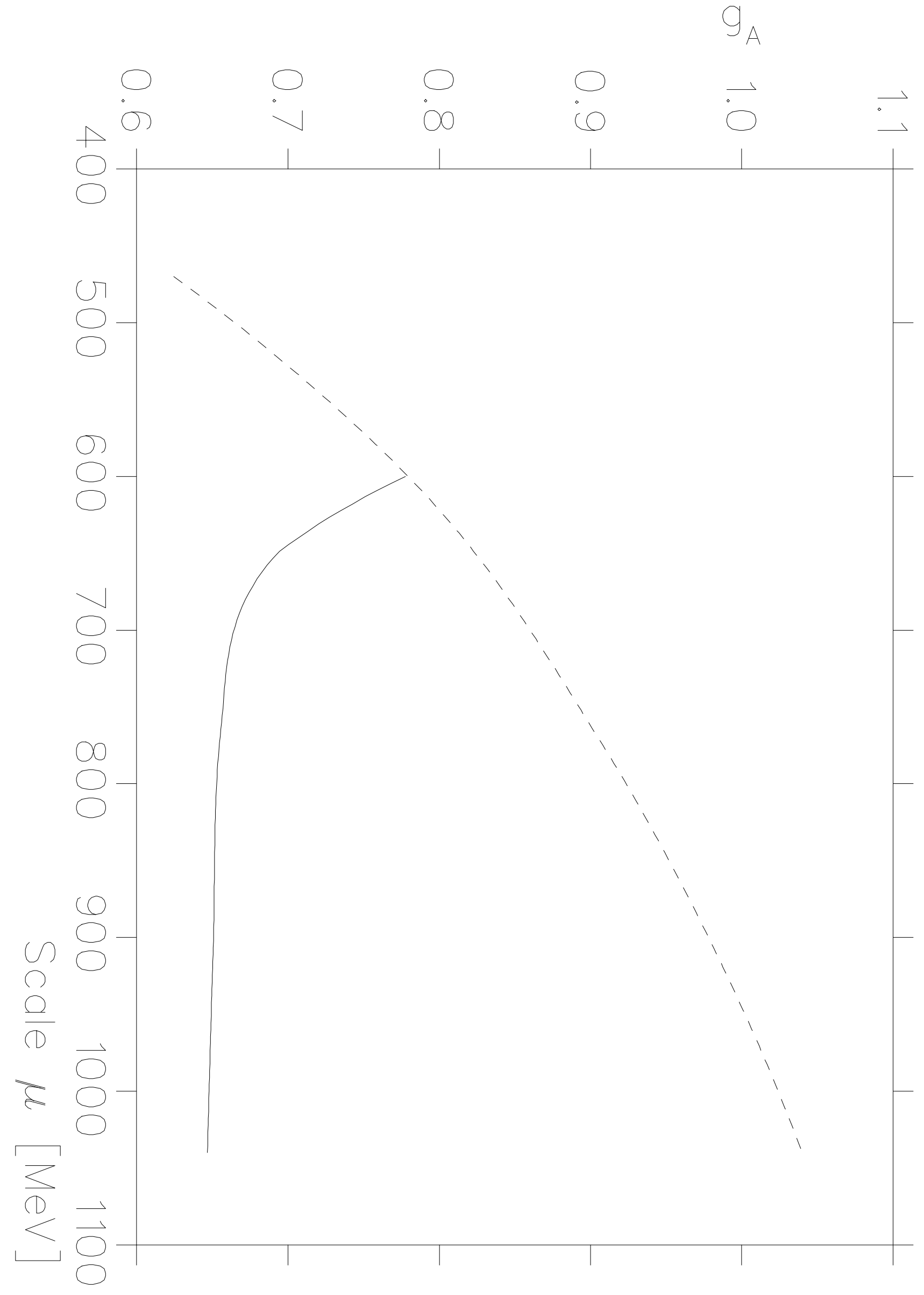




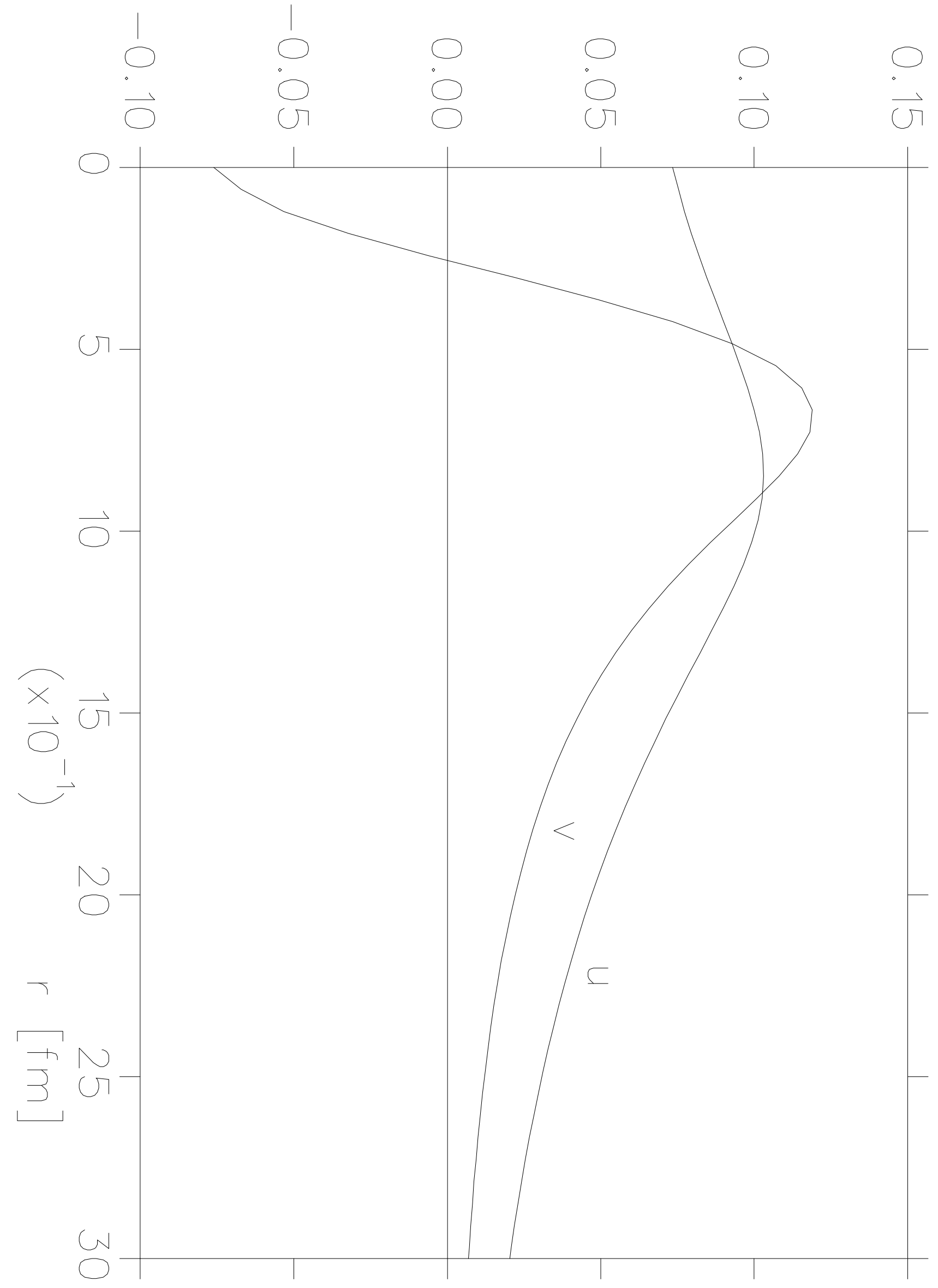




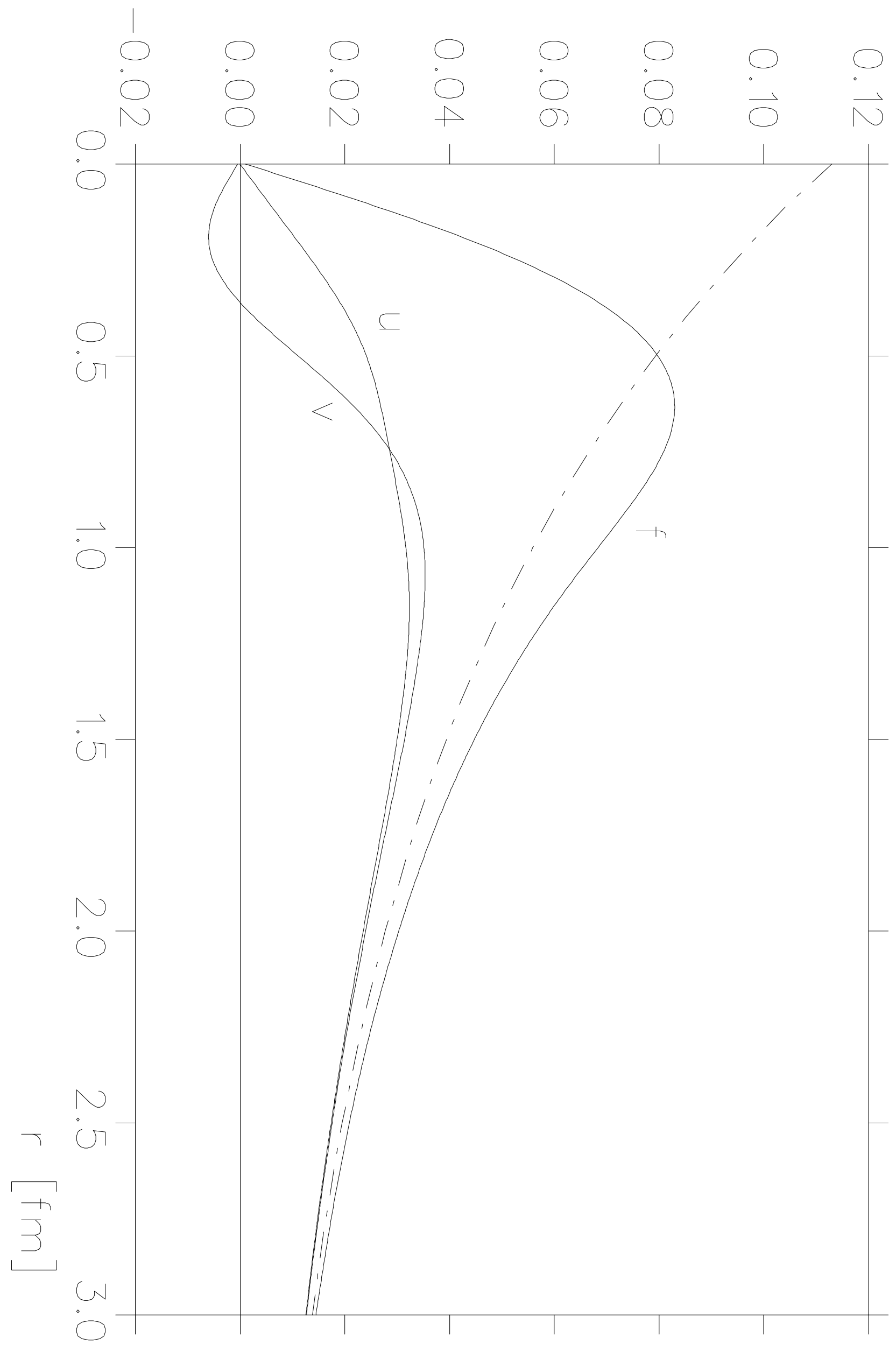

